\title{
The Kepler Conjecture
}

\author{
Thomas C. Hales
}

March 8, 2002

\section{Abstract}

We present the final part of the proof of the Kepler Conjecture.

\section{Overview}

This section describes the structure of the proof of the Kepler Conjecture.

Theorem 1.1. (The Kepler Conjecture) No packing of congruent balls in Euclidean three space has density greater than that of the face-centered cubic packing.

This density is $\pi / \sqrt{18} \approx 0.74$.

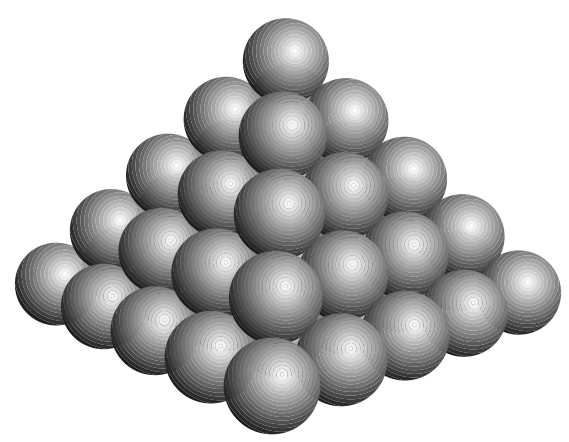

Figure 1: The face-centered cubic packing

The proof of this result is scattered throughout several papers. (Every article in the bibliography will be required.) Here, we describe the toplevel outline of the proof and give references to the sources of the details of the proof.

By a packing, we mean an arrangement of balls that are non-overlapping in the sense that the interiors of the balls are pairwise disjoint. Consider a packing of congruent balls in Euclidean three space. There is no harm in assuming that all the balls have unit radius. The density of a packing does not decrease when balls are added to the packing.
Thus, to answer a question about the greatest possible density we may add non-overlapping balls until there is no room to add further balls. Such a packing will be said to be saturated.

Let $\Lambda$ be the set of centers of the balls in a saturated packing. Our choice of radius for the balls implies that any two points in $\Lambda$ have distance at least 2 from each other. We call the points of $\Lambda$ vertices. Let $B(x, r)$ denote the ball in Euclidean three space at center $x$ and radius $r$. Let $\delta(x, r, \Lambda)$ be the finite density, defined by the ratio of $A(x, r, \Lambda)$ to the volume of $B(x, r)$, where $A(x, r, \Lambda)$ is defined as the volume of the intersection with $B(x, r)$ of the union of all balls in the packing. Set $\Lambda(x, r)=\Lambda \cap B(x, r)$.

Recall that the Voronoi cell $\Omega(v)$ around a vertex $v \in \Lambda$ is the set of points closer to $v$ than to any other ball center. The volume of each Voronoi cell in the face-centered cubic packing is $\sqrt{32}$. This is also the volume of each Voronoi cell in the hexagonalclose packing.

Let $a: \Lambda \rightarrow \mathbb{R}$ be a function. We say that $a$ is negligible if there is a constant $C_{1}$ such that for all $r \geq 1$, we have

$$
\sum_{v \in \Lambda(x, r)} a(v) \leq C_{1} r^{2} .
$$

We say that the function $a$ is fcc-compatible if for all $v \in \Lambda$ we have the inequality

$$
\sqrt{32} \leq \operatorname{vol}(\Omega(v))+a(v) .
$$

Lemma 1.2. If there exists a negligible fcccompatible function $a: \Lambda \rightarrow \mathbb{R}$ for a saturated packing $\Lambda$, then there exists a constant $C$ such that for all $r \geq 1$, we have

$$
\delta(x, r, \Lambda) \leq \pi / \sqrt{18}+C / r .
$$

Proof. The numerator $A(x, r, \Lambda)$ of $\delta(x, r, \Lambda)$ is at most the product of the volume of a ball $4 \pi / 3$ with the number $|\Lambda(x, r+1)|$ of balls intersecting $B(x, r)$. Hence

$$
A(x, r, \Lambda) \leq|\Lambda(x, r+1)| 4 \pi / 3 .
$$


In a saturated packing each Voronoi cell is contained in a ball of radius 2 centered at the center of the cell. The volume of the ball $B(x, r+3)$ is at least the combined volume of Voronoi cells lying entirely in the ball. This observation, combined with fcc-compatibility and negligibility, gives

$$
\begin{aligned}
\sqrt{32}|\Lambda(x, r+1)| & \leq \sum_{v \in \Lambda(x, r+1)}(a(v)+\operatorname{vol}(\Omega(v))) \\
& \leq C_{1}(r+1)^{2}+\operatorname{vol} B(x, r+3) \\
& \leq C_{1}(r+1)^{2}+(1+3 / r)^{3} \operatorname{vol} B(x, r)
\end{aligned}
$$

Divide through by $\operatorname{vol} B(x, r)$ and eliminate $|\Lambda(x, r+1)|$ between Inequality (1) and Inequality (2) to get

$$
\delta(x, r, \Lambda) \leq \frac{\pi}{\sqrt{18}}(1+3 / r)^{3}+C_{1} \frac{(r+1)^{2}}{r^{3} \sqrt{32}} .
$$

The result follows for an appropriately chosen constant $C$.

Remark 1.3. We take the precise meaning of the Kepler Conjecture to be a bound on the essential supremum of the function $\delta(x, r)$ as $r$ tends to infinity. Lemma 1.2 implies that the essential supremum of $\delta(x, r, \Lambda)$ is bounded above by $\pi / \sqrt{18}$, provided a negligible fcc-compatible function can be found. The strategy will be to define a negligible function, and then to solve an optimization problem in finitely many variables to establish that it is fcc-compatible.

The paper [2] defines a compact topological space $X$ and a continuous function $\sigma$ on that space.

The topological space $X$ is directly related to packings. If $\Lambda$ is a saturated packing, then there is a geometric object $D(v, \Lambda)$ constructed around each vertex $v \in \Lambda . D(v, \Lambda)$ depends on $\Lambda$ only through the vertices in $\Lambda$ at distance at most 4 from $v$. The objects $D(v, \Lambda)$ are called decomposition stars, and the space of all decomposition stars is precisely $X$.

The following constants arise in that paper.

Let $\delta_{\text {tet }}$ be the packing density of a regular tetrahedron. That is, let $S$ be a regular tetrahedron of edge length 2. Let $B$ the part of $S$ that lies within distance 1 of some vertex. Then $\delta_{t e t}$ is the ratio of the volume of $B$ to the volume of $S$. We have $\delta_{\text {tet }}=\sqrt{8} \arctan (\sqrt{2} / 5)$.

Let $\delta_{\text {oct }}$ be the packing density of a regular octahedron of edge length 2, again constructed as the ratio of the volume of points within distance 1 of a vertex to the volume of the octahedron.
The density of the face-centered cubic packing is a weighted average of these two ratios

$$
\frac{\pi}{\sqrt{18}}=\frac{\delta_{t e t}}{3}+\frac{2 \delta_{o c t}}{3} .
$$

This determines the exact value of $\delta_{\text {oct }}$ in terms of $\delta_{\text {tet }}$. We have $\delta_{\text {oct }} \approx 0.72$.

Let $p t=-\pi / 3+\sqrt{2} \delta_{\text {tet }} \approx 0.05537$

The following conjecture is made in [2]

Conjecture 1.4. The maximum of $\sigma$ on $X$ is the constant $8 p t \approx 0.442989$.

Lemma 1.5. An affirmative answer to Conjecture 1.4 implies the existence of a negligible fcccompatible function for every saturated packing $\Lambda$.

Proof. For any saturated packing $\Lambda$ define a function $a: \Lambda \rightarrow \mathbb{R}$ by

$-\sigma(D(v, \Lambda)) /\left(4 \delta_{\text {oct }}\right)+4 \pi /\left(3 \delta_{\text {oct }}\right)=\operatorname{vol}(\Omega(v))+a(v)$.

Negligibility follows from [2, Prop. 3.14 (proof)]. The upper bound of $8 p t$ gives a lower bound

$$
-8 p t /\left(4 \delta_{\text {oct }}\right)+4 \pi /\left(3 \delta_{\text {oct }}\right) \leq \operatorname{vol}(\Omega(v))+a(v) .
$$

The left-hand side of this inequality evaluates to $\sqrt{32}$, and this establishes fcc-compatibility.

Theorem 1.6. Conjecture 1.4 is true. That is, the maximum of the function $\sigma$ on the topological space $X$ of all decomposition stars is 8 t.

Theorem 1.6, Lemma 1.5, and Lemma 1.2 combine to give a proof of the Kepler Conjecture 1.1

Let $t_{0}=1.255\left(2 t_{0}=2.51\right)$. This is a parameter that is used for truncation throughout the series of papers on the Kepler Conjecture. The significance of this particular choice of truncation parameter will be explained elsewhere.

Let $U(v, \Lambda)$ be the set of vertices in $\Lambda$ at distance at most $2 t_{0}$ from $v$. From a decomposition star $D(v, \Lambda)$ it is possible to recover $U(v, \Lambda)$ (at least up to Euclidean translation: $U \mapsto U+y$, for $y \in \mathbb{R}^{3}$ ). We can completely characterize the decomposition stars at which the maximum of $\sigma$ is attained.

Theorem 1.7. Let $D$ be a decomposition star at which the maximum 8 pt is attained. Then the set $U(D)$ of vectors at distance at most $2 t_{0}$ from the center has cardinality 12 . Up to Euclidean motion, $U(D)$ is the kissing arrangement of the 12 balls around a central ball in the face-centered cubic packing or hexagonal-close packing. 


\subsection{Outline of proofs}

To prove Theorems 1.6 and 1.7, we wish to show that there is no counterexample. That is, we wish to show that there is no decomposition star $D$ with value $\sigma(D)>8 \mathrm{pt}$. We reason by contradiction, assuming the existence of such a decomposition star. With this in mind, we call $D$ a contravening decomposition star, if

$$
\sigma(D) \geq 8 p t
$$

In much of what follows we will tacitly assume that every decomposition star under discussion is a contravening one. Thus, when we say that no decomposition stars exist with a given property, it should be interpreted as saying that no such contravening decomposition stars exist.

To each contravening decomposition star, we associate a (combinatorial) plane graph. A restrictive list of properties of plane graphs is described in Section 2.3. Any plane graph satisfying these properties is said to be tame. All tame plane graphs have been classified. (There are several thousand, up to isomorphism.) Theorem 2.1, asserts that the plane graph attached to each contravening decomposition star is tame. By the classification of such graphs, this reduces the proof of the Kepler Conjecture to the analysis of the decomposition stars attached to the finite explicit list of tame plane graphs.

A few of the tame plane graphs are of particular interest. Every decomposition star attached to the face-centered cubic packing gives the same plane graph (up to isomorphism). Call it $G_{f c c}$. Likewise, every decomposition star attached to the hexagonal-close packing gives the same plane graph $G_{h c p}$. Let $X_{\text {crit }}$ be the set of decomposition stars $D$ such that the set $U(D)$ of vertices is the kissing arrangement of the 12 balls around a central ball in the face-centered cubic or hexagonal-close packing. There are only finitely many orbits of $X_{\text {crit }}$ under the group of Euclidean motions.
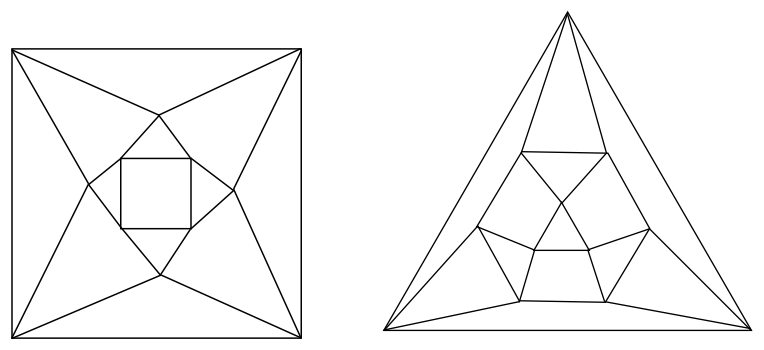

Figure 2: The plane graphs $G_{f c c}$ and $G_{h c p}$
In [2, Lemma 3.13], the necessary local analysis is carried out to prove the following local optimality.

Lemma 1.8. A decomposition star whose plane graph is $G_{f c c}$ or $G_{h c p}$ has score at most 8 pt, with equality precisely when the decomposition star belongs to $X_{\text {crit }}$.

In light of this result, we prove 1.6 and 1.7 by proving that any decomposition star whose graph is tame and not equal to $G_{f c c}$ or $G_{h c p}$ is not contravening

There is one more tame plane graph that is particularly troublesome. It is the graph $G_{\text {pent }}$ obtained from the pictured configuration of twelve balls tangent to a given central ball (Figure 3). (Place a ball at the north pole, another at the south pole, and then form two pentagonal rings of five balls.) This case requires individualized attention. S. Ferguson proves in [7] that if $D$ is any decomposition star with this graph, then $\sigma(D)<8 p t$.

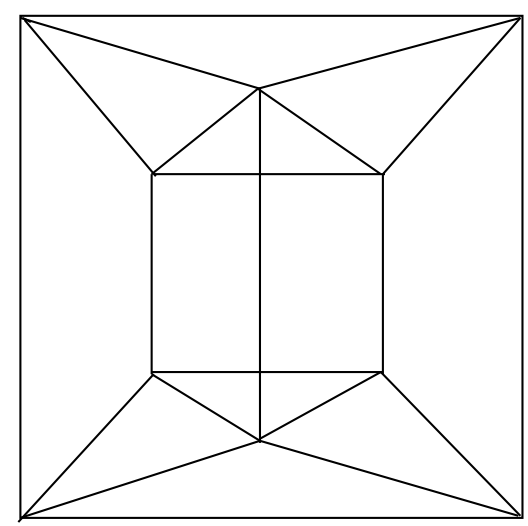

Figure 3: The plane graph $G_{\text {pent }}$

To eliminate the remaining cases, more-or-less generic arguments can be used. A linear program is attached to each tame graph $G$. The linear program can be viewed as a linear relaxation of the nonlinear optimization problem of maximizing $\sigma$ over all decomposition stars with a given tame graph $G$. Because it is obtained by relaxing the constraints on the nonlinear problem, the maximum of the linear problem is an upper bound on the maximum of the original nonlinear problem. Whenever the linear programming maximum is less than $8 p t$, it can be concluded that there is no contravening decomposition star with the given tame graph $G$. This linear programming approach eliminates most tame graphs.

When a single linear program fails to give the desired bound, it is broken into a series of linear 
programming bounds, by branch and bound techniques. For every tame plane graph $G$ other than $G_{h c p}, G_{f c c}$, and $G_{\text {pent }}$, we produce a series of linear programs that establish that there is no contravening decomposition star with graph $G$. When every face of the plane graph is a triangle or quadrilateral, this is accomplished in [5]. The general case is completed in the final sections of this paper.

\subsection{Organization of the Paper}

This paper has been written in such a way that several sections can be skipped without disrupting the expository flow. To get a general overview of the paper, Sections 1, 2, 4, and 10 may be read. To get a somewhat more detailed view of the results and methods of proof, Sections 1, 2, 3, 4, 5, 10, 11, and the first two appendices may be read.

The paper has been written so as to be substantially independent of the other papers in the series. An appendix gives a summary of the results of [6] in a form that is suited to the purposes of this paper.

A historical account of the Kepler Conjecture can be found at [1]. An introduction to the ideas of the proof can be found in [11]. An introduction to the algorithms can be found at [9]. Speculation on a second-generation design of a proof can be found in [9] and [12].

\section{Tame Plane Graphs}

We give a succinct statement of properties that hold of all plane graphs considered in the proof of the Kepler conjecture. Such graphs are said to be tame. A list of all tame graphs has been generated by computer.

\subsection{Basic Definitions}

An $n$-cycle is a finite set $C$ of cardinality $n$,together with a cyclic permutation $s$ of $C$. We write $s$ in the form $v \mapsto s(v, C)$, for $v \in C$. The element $s(v, C)$ is called the successor of $v$ (in $C$ ). A cycle is a $n$-cycle for some natural number $n$. By abuse of language, we often identify $C$ with the cycle. The natural number $n$ is the length of the cycle.

(We may assume that all vertices of all graphs lie in some large finite set of vertices $\Omega$, if we wish to arrange that only finitely many graphs occur in this discussion.)
Let $G$ be a nonempty finite set of cycles (called faces) of length at least 3 . The elements of faces are called the vertices of $G$. An unordered pair of vertices $\{v, w\}$ such that one element is the successor of the other in some face is called an edge. The vertices $v$ and $w$ are then said to be adjacent. The set $G$ is a plane graph if three conditions hold.

1. If an element $v$ has successor $w$ in some face $F$, then there is a unique face (call it $s^{\prime}(F, v)$ ) in $G$ for which $v$ is the successor of $w$. (Thus, $v=s\left(w, s^{\prime}(F, v)\right)$, and each edge occurs twice with opposite orientation.)

2. For each vertex $v$, the function $F \mapsto s^{\prime}(F, v)$ is a cyclic permutation of the set of faces containing $v$.

3. Euler's formula holds relating the number of vertices $V$, the number of edges $E$, and the number of faces $F$ :

$$
V-E+F=2
$$

(The set of vertices and edges of a plane graph form a planar graph in the usual graph-theoretic sense of admitting an embedding into the plane.)

Let len be the length function on faces. Let $\operatorname{tri}(v)$ be the number of triangles containing a vertex $v$. Faces of length 3 are called triangles, those of length 4 are called quadrilaterals, and so forth. An face of length at least 5 is called an exceptional face.

Two plane graphs are properly isomorphic if there is a bijection of vertices inducing a bijection of faces. For each plane graph, there is an opposite plane graph $G^{o p}$ obtained by reversing the cyclic order of vertices in each face. A plane graph $G$ is isomorphic to another if $G$ or $G^{o p}$ is properly isomorphic to the other.

The degree of a vertex is the number of faces it belongs to. An $n$-circuit in $G$ is a cycle $C$ in the vertex-set of $G$, such that for every $v \in C$, it forms an edge in $G$ with its successor: that is, $(v, s(v, C))$ is an edge of $G$. In a plane graph $G$ we have a combinatorial form of the Jordan curve theorem: each $n$-circuit determines a partition of $G$ into two sets of faces.

The type of a vertex is defined to be a triple of non-negative integers $(p, q, r)$, where $p$ is the number of triangles containing the vertex, $q$ is the number of quadrilaterals containing it, and $r$ is the number of exceptional faces. When $r=0$, we abbreviate the type to the ordered pair $(p, q)$. 


\subsection{Weight Assignments}

We call the constant 14.8 , which arises repeatedly in this section, the target.

Define $a: \mathbb{N} \rightarrow \mathbb{R}$ by

$$
a= \begin{cases}14.8 & n=0,1,2, \\ 1.4 & n=3, \\ 1.5 & n=4, \\ 0 & \text { otherwise }\end{cases}
$$

Define $b: \mathbb{N} \times \mathbb{N} \rightarrow \mathbb{R}$ by $b(p, q)=14.8$, except for the values in the following table (with the understanding that $x=14.8$ ):

$\begin{array}{cccccc} & q=0 & 1 & 2 & 3 & 4 \\ p=0 & x & x & x & 7.135 & 10.649 \\ 1 & x & x & 6.95 & 7.135 & x \\ 2 & x & 8.5 & 4.756 & 12.981 & x \\ 3 & x & 3.642 & 8.334 & x & x \\ 4 & 4.139 & 3.781 & x & x & x \\ 5 & 0.55 & 11.22 & x & x & x \\ 6 & 6.339 & x & x & x & x\end{array}$

Define $c: \mathbb{N} \rightarrow \mathbb{R}$ by

$$
c= \begin{cases}1 & n=3 \\ 0 & n=4 \\ -1.03 & n=5 \\ -2.06 & n=6 \\ -3.03 & \text { otherwise }\end{cases}
$$

Define $d: \mathbb{N} \rightarrow \mathbb{R}$ by

$$
d= \begin{cases}0 & n=3 \\ 2.378 & n=4 \\ 4.896 & n=5 \\ 7.414 & n=6 \\ 9.932 & n=7 \\ 10.916 & n=8 \\ 14.8 & \text { otherwise. }\end{cases}
$$

A set $V$ of vertices is called a separated set of vertices if the following four conditions hold.

1. For every vertex in $V$ there is an exceptional face containing it.

2. No two vertices in $V$ are adjacent.

3. No two vertices in $V$ lie on a common quadrilateral.

4. Each vertex in $V$ has degree 5 .
A weight assignment of a plane graph $G$ is a function $w: G \rightarrow \mathbb{R}$ taking values in the set of non-negative real numbers. A weight assignment is admissible if the following properties hold:

1. If the face $F$ has length $n$, then $w(F) \geq d(n)$

2. If $v$ has type $(p, q)$, then

$$
\sum_{v \in F} w(F) \geq b(p, q) .
$$

3. Let $V$ be any set of vertices of type $(5,0)$. If the cardinality of $V$ is $k \leq 4$, then

$$
\sum_{V \cap F \neq \emptyset} w(F) \geq 0.55 k \text {. }
$$

4. Let $V$ be any separated set of vertices. Then

$$
\sum_{V \cap F \neq \emptyset}(w(F)-d(\operatorname{len}(F))) \geq \sum_{v \in V} a(\operatorname{tri}(v)) .
$$

The sum $\sum_{F} w(F)$ is called the total weight of $w$.

\subsection{Plane Graph Properties}

We say that a plane graph is tame if it satisfies the following conditions.

1. The length of each face is (at least 3 and) at most 8 .

2. Every 3-circuit is a face or the opposite of a face.

3. Every 4-circuit surrounds one of the cases illustrated in Figure 4.

4. The degree of every vertex is (at least 2 and) at most 6 .

5. If a vertex is contained in an exceptional face, then the degree of the vertex is at most 5 .

6.

$$
\sum_{F} c(l e n(F)) \geq 8
$$

7. There exists an admissible weight assignment of total weight less than the target, 14.8.

It follows from the definitions that the abstract vertex-edge graph of $G$ has no loops or multiple joins. Also, by construction, every vertex lies in at least two faces. Property 6 asserts that the graph has at least eight triangles. 

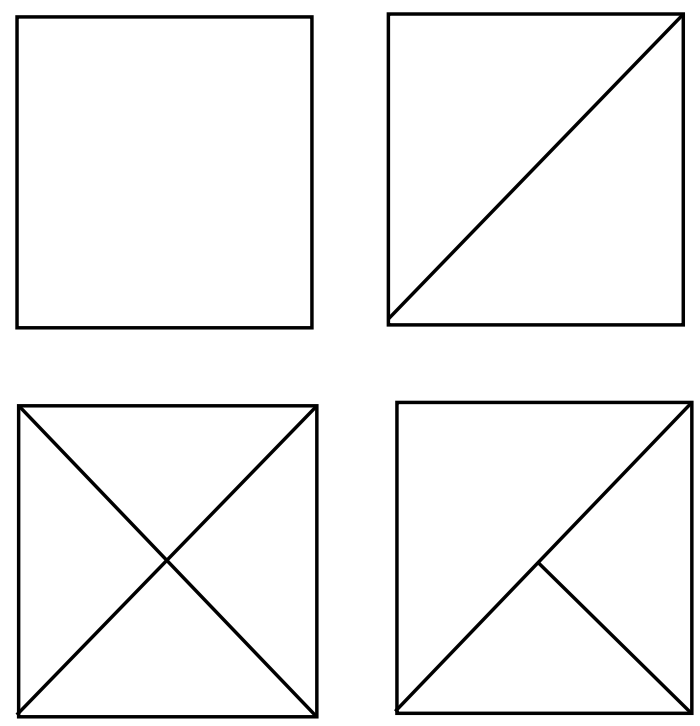

Figure 4: Tame 4-circuits

\subsection{Classification of Tame Plane Graphs}

A list of several thousand plane graphs appears at [8].

Theorem 2.1. Every tame plane graph is isomorphic to a plane graph in this list.

\section{Proof of Classification}

The results of this section are not needed except in the proof of Theorem 2.1.

Computers are used to generate a list of all tame plane graphs and to check them against the archive of tame plane graphs. We will describe a finite state machine that produces all tame plane graphs. This machine is not particularly efficient, and so we also include a description of pruning strategies that prevent a combinatorial explosion of possibilities.

\subsection{Basic Definitions}

A partial plane graph is a plane graph with additional data: every face is marked as "complete" or "incomplete." We call a face complete or incomplete according to the markings. We require the following condition.

- No two incomplete faces share an edge.
Each unmarked plane graph is identified with the marked plane graph in which every face is complete. We represent a partial plane graph graphically by deleting one face (the face at infinity) and drawing the others and shading those that are complete.

A patch is a partial plane graph $P$ with two distinguished faces $F_{1}$ and $F_{2}$, such that the following hold.

- Every vertex of $P$ lies in $F_{1}$ or $F_{2}$.

- The face $F_{2}$ is the only complete face.

- $F_{1}$ and $F_{2}$ share an edge.

- Every vertex of $F_{2}$ that is not in $F_{1}$ has degree 2 .

$F_{1}$ and $F_{2}$ will be referred to as the distinguished incomplete and the distinguished complete faces, respectively.

Patches can be used to modify a partial plane graph as follows. Let $F$ be an incomplete face of length $n$ in a partial plane graph $G$. Let $P$ be a patch whose incomplete distinguished face $F_{1}$ has length $n$. Replace $P$ with a properly isomorphic patch $P^{\prime}$ in which the image of $F_{1}$ is equal to $F^{o p}$ and in which no other vertex of $P^{\prime}$ is a vertex of $G$. Then

$$
G^{\prime}=\left\{F^{\prime} \in G \cup P^{\prime}: F^{\prime} \neq F^{o p}, F^{\prime} \neq F\right\}
$$

is a partial plane graph. Intuitively, we cut away the faces $F$ and $F_{1}$ from their plane graphs, and glue the holes together along the boundary (Figure 5). (It is immediate that the Condition 3.1 in the definition of partial plane graphs is maintained by this process.) There are $n$ distinct proper ways of identifying $F_{1}$ with $F^{o p}$ in this construction, and we let $\phi$ be this identification. The isomorphism class of $G^{\prime}$ is uniquely determined by the isomorphism class of $G$, the isomorphism class of $P$, and $\phi$ (ranging over proper bijections $\phi: F_{1} \mapsto F^{o p}$ ).

\subsection{A Finite State Machine}

For a fixed $N$ we define a finite state machine as follows. The states of the finite state machine are isomorphism classes of partial plane graphs $G$ with at most $N$ vertices. The transitions from one state $G$ to another are isomorphism classes of pairs $(P, \phi)$ where $P$ is a patch, and $\phi$ pairs an incomplete face of $G$ with the distinguished incomplete face of $P$. However, we exclude a transition $(P, \phi)$ at a state 

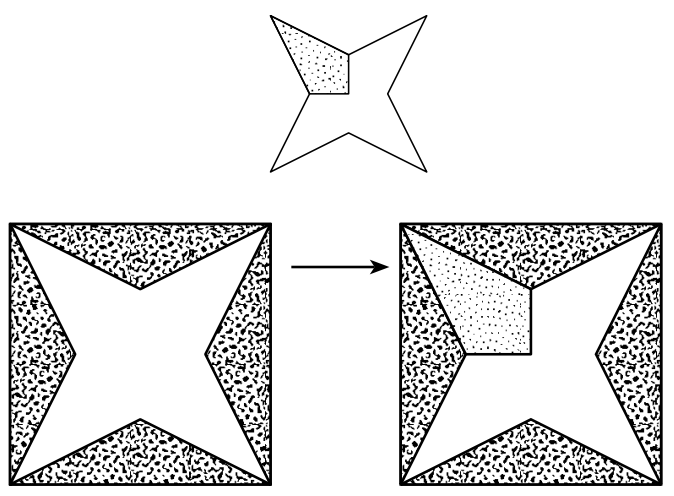

Figure 5: Patching a plane graph

if the resulting partial plane graphs contains more than $N$ vertices. Figure 5 shows two states and a transition between them.

The initial states $I_{n}$ of the finite state machine are defined to be the isomorphism classes of partial plane graphs with two faces:

$$
\{(1,2, \ldots, n),(n, n-1, \ldots, 1)\}
$$

where $n \leq N$, one face is complete, and the other is incomplete. In other words, they are patches with exactly two faces.

A terminal state of this finite state machine is one in which every face is complete. By construction, these are (isomorphism classes of) plane graphs with at most $N$ vertices.

Lemma 3.1. Let $G$ be a plane graph with at most $N$ vertices. Then its state in the machine is reachable from an initial state through a series of transitions.

Proof. Pick an face in $G$ of length $n$ and identify it with the complete face in the initial state $I_{n}$. At any stage at state $G^{\prime}$, we have an identification of all of the vertices of the plane graph $G^{\prime}$ with some of the vertices of $G$, and an identification of all of the complete faces of $G^{\prime}$ with some of the faces of $G$ (all faces of $G$ are complete). Pick an incomplete face $F$ of $G^{\prime}$ and an oriented edge along that face. We let $F^{\prime}$ be the complete face of $G$ with that edge, with the same orientation on that edge as $F$. Create a patch with distinguished faces $F_{1}=F^{o p}$ and $F_{2}=F^{\prime} . \quad\left(F_{1}\right.$ and $F_{2}$ determine the patch up to isomorphism.) It is immediate that the conditions defining a patch are fulfilled. Continue in this way until a graph isomorphic to $G$ is reached.

Remark 3.2. It is an elementary matter to generate all patches $P$ such that the distinguished faces have given lengths $n$ and $m$. Patching is also entirely algorithmic, and thus by following all paths through the finite state machine, we obtain all plane graphs with at most $N$ vertices.

\subsection{Pruning Strategies}

Although we reach all graphs in this manner, it is not computationally efficient. We introduce pruning strategies to increase the efficiency of the search. We can terminate our search along a path through the finite state machine, if we can determine:

1. Every terminal graph along that path violates one of the defining properties of tameness, or

2. An isomorphic terminal graph will be reached by some other path that will not be terminated early.

Here are some pruning strategies of the first type (1). They are immediate consequences of the conditions of the defining properties of tameness.

- If the current state contains an incomplete face of length 3 , then eliminate all transitions, except for the transition that carries the partial plane graph to a partial plane graph that is the same in all respects, except that the face has become complete.

- If the current state contains an incomplete face of length 4 , then eliminate all transitions except those that lead to the possibilities of Section 2.3, Property 3, where in Property 3 each depicted face is interpreted as being complete.

- Remove all transitions with patches whose complete face has length greater than 8 .

- It is frequently possible to conclude from the examination of a partial plane graph that no matter what the terminal position, any admissible weight assignment will give total weight greater than the target (14.8). In such cases, the all transitions out of the partial plane graph can be pruned.

To take a simple example of the last item, we observe that weights are always non-negative, and that the weight of a complete face of length $n$ is at least $d(n)$. Thus, if there are complete faces $F_{1}, \ldots, F_{k}$ of lengths $n_{1}, \ldots, n_{k}$, then any admissible weight assignment has total weight at least $\sum_{i=1}^{k} d\left(n_{i}\right)$. If this number is at least the target, 
then no transitions out of that state need be considered.

More generally, we can apply all of the inequalities in the definition of admissible weight assignment to the complete portion of the partial plane graph to obtain lower bounds. However, we must be careful, in applying Property 4 of admissible weight assignments, because vertices that are not adjacent at an intermediate state may become adjacent in the complete graph. Also, vertices that do not lie together in a quadrilateral at an intermediate state may do so in the complete graph.

Here are some pruning strategies of the second type (2).

- At a given state it is enough to fix one incomplete face and one edge of that face and then to follow only the transitions that patch along that face and add a complete face along that edge. (This is seen from the proof of Lemma 3.1.)

- In leading out from the initial state $I_{n}$, it is enough to follow paths in which every added complete face has length at most $n$. (A graph with a face of length $m$, for $m>n$, will be also be found downstream from $I_{m}$.)

- Make a list of all type $(p, q)$ with $b(p, q)<14.8$. Remove the initial states $I_{3}$ and $I_{4}$, and create new initial states $I_{p, q}\left(I_{p, q}^{\prime}, I_{p, q}^{\prime \prime}\right.$, etc. $)$ in the finite state machine. Define the state $I_{p, q}$ to be one consisting of $p+q+1$ faces, with $p$ complete triangles and $q$ complete quadrilaterals all meeting at a vertex (and one other incomplete face away from $v$ ). (If there is more than one way to arrange $p$ triangles and $q$ quadrilaterals, create states $I_{p, q}, I_{p, q}^{\prime}, I_{p, q}^{\prime \prime}$, for each possibility. See Figure 6.) Put a linear order on states $I_{p, q}$. In state transitions downstream from $I_{p, q}$ disallow any transition that creates a vertex of type $\left(p^{\prime}, q^{\prime}\right)$, for any $\left(p^{\prime}, q^{\prime}\right)$ preceding $(p, q)$ in the imposed linear order.
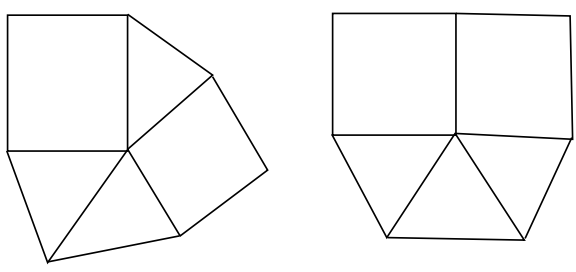

Figure 6: States $I_{3,2}$ and $I_{3,2}^{\prime}$
This last pruning strategy is justified by the following lemma, which classifies vertices of type $(p, q)$.

Lemma 3.3. Let $A$ and $B$ be triangular or quadrilateral faces that have at least 2 vertices in common in a tame graph. Then the faces have exactly two vertices in common, and an edge is shared by the two faces.

Proof. Exercise. Some of the configurations that must be ruled out are shown in Figure 7 . Some properties that are particularly useful for the exercise are Properties 2 and 3 of tameness, and Property 2 of admissibility.

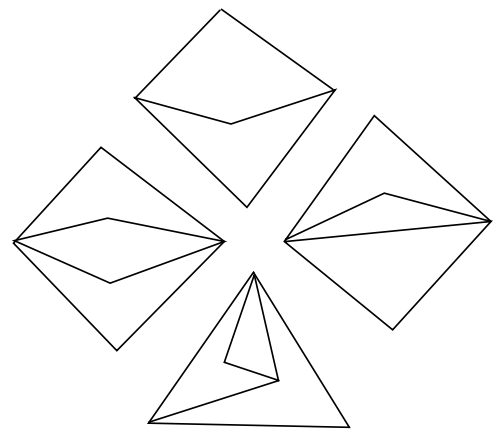

Figure 7: Some impossibilities

Once a terminal position is reached it is checked to see whether it satisfies all the properties of tameness.

Duplication is removed among isomorphic terminal plane graphs. It is not an entirely trivial procedure for the computer to determine whether there exists an isomorphism between two plane graphs. This is accomplished by computing a numerical invariant of a vertex that depends only on the local structure of the vertex. If two plane graphs are properly isomorphic then the numerical invariant is the same at vertices that correspond under the proper isomorphism. If two graphs have the number of vertices with the same numerical invariants, they become candidates for an isomorphism. All possible numerical-invariant preserving bijections are attempted until an proper isomorphism is found, or until it is found that none exist. If there is no proper isomorphism, the same procedure is applied to the opposite plane graph to find any possible orientation-reversing isomorphism.

This same isomorphism-producing algorithm is used to match each terminal graph with a graph in the archive. It is found that each terminal graph matches with one in the archive. (The archive was 
originally obtained by running the finite state machine and making a list of all the terminal states up to isomorphism that satisfy the given conditions.)

In this way Theorem 2.1 is proved.

\section{From Stars to Graphs}

\subsection{Plane Graphs}

A plane graph $G$ is attached to every contravening decomposition star as follows. For simplicity, take the decomposition star to be attached to a ball of the packing, centered at the origin. From the decomposition star $D$, it is possible to determine the coordinates of the set $U(D)$ of vertices at distance at most $2 t_{0}$ from the origin.

If we draw a geodesic arc on the unit sphere at the origin with endpoints at the projections of $v_{1}$ and $v_{2}$ for every pair of vertices $v_{1}, v_{2} \in U(D)$ such that $\left|v_{1}\right|,\left|v_{2}\right|,\left|v_{1}-v_{2}\right| \leq 2 t_{0}$, we obtain a plane graph that breaks the unit sphere into regions called standard regions. (The arcs do not meet except at endpoints [3, Lemma 3.10].) Each standard region is defined as the closure in the unit sphere of a connected component of the unit sphere with all arcs removed.

For a given standard region, we consider the arcs forming its boundary together with the arcs that are internal to the standard region. We consider the points on the unit sphere formed by the endpoints of the arcs, together with the projections to the unit sphere of vertices in $U$ whose projection lies in the interior of the region.

Remark 4.1. The system of arcs and vertices associated with a standard region in a contravening example must be a polygon, or one of the following configurations of Figure 8 (see [6, Corollary 4.4]).

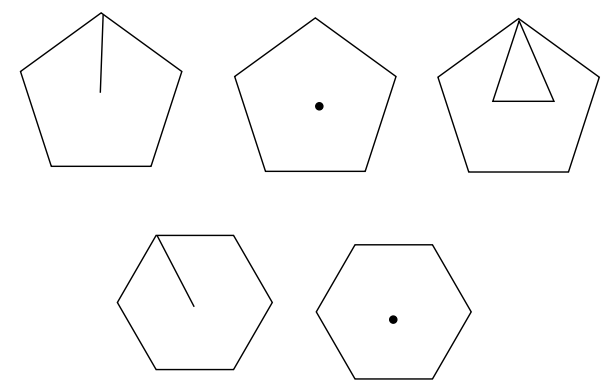

Figure 8: Non-polygonal standard regions
Remark 4.2. Observe that one case is bounded by a triangle and a pentagon, and that the others are bounded by a polygon. Replacing the trianglepentagon arrangement with the bounding pentagon and replacing the others with the bounding polygon, we obtain a partition of the sphere into simple polygons. Each of these polygons is a single standard region, except in the triangle-pentagon case (Figure 9), which is a union of two standard regions (a triangle and a eight-sided region).

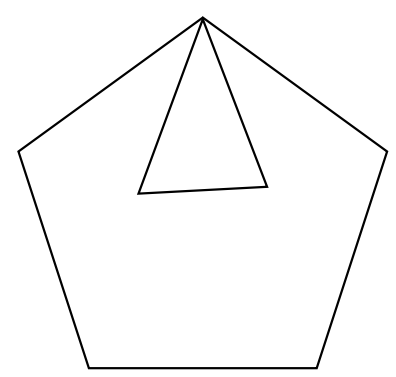

Figure 9: An aggregate forming a pentagon

Remark 4.3. To simplify further, if we have an arrangement of six standard regions around a vertex formed from five triangles and one pentagon, we replace it with the bounding octagon (or hexagon). See Figure 10. (It will be shown in Lemma 6.3 and Section 9.2 that there is at most one such configuration in the standard decomposition of a contravening decomposition star, so we will not worry here about how to treat the case of two overlapping configurations of this sort.)

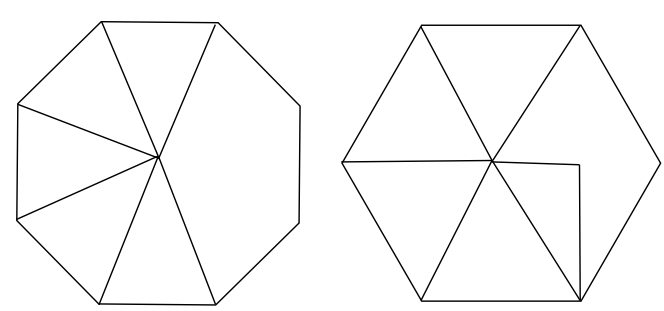

Figure 10: Degree 6 aggregates

In summary, we have a plane graph that is approximately that given by the standard regions of the decomposition star, but simplified to a bounding polygon when one of the configurations of Remarks 4.2 and 4.3 occur. We refer to the combination of standard regions into a single face of the graph as aggregation. We call it the plane graph $G=G(D)$ attached to a contravening decomposition star $D$. 
Proposition 5.1 will show the vertex set $U$ is nonempty and that the graph $G(D)$ is non-empty.

When we refer to the plane graph in this manner, we mean the combinatorial plane graph in the sense of Section 2, as opposed to the embedded metric graph on the unit sphere formed from the system of geodesic arcs. Given a vertex $v$ in $G(D)$, there is a uniquely determined vertex $v(D)$ of $U(D)$ whose radial projection to the unit sphere determines $v$. We call $v(D)$ the corner in $U(D)$ over $v$.

By definition, the plane graphs in Section 2 do not have loops or multiple joins. The edges of $G(D)$ are defined by triangles whose sides vary between lengths 2 and $2 t_{0}$. The angles of such a triangle are strictly less than $\pi$. This implies that the edges of the metric graph on the unit sphere always have arc-length strictly less than $\pi$. In particular, the endpoints are never antipodal. A loop on the combinatorial graph corresponds to a edge on the metric graph that is a closed geodesic. A multiple join on the combinatorial graph corresponds on the metric graph to a pair of points joined by multiple minimal geodesics, that is, a pair of antipodal points on the sphere. By the arc-length constraints on edges in the metric graph, there are no loops or multiple joins in the combinatorial graph $G(D)$.

In Definition 2.3, a plane graph satisfying a certain restrictive set of properties is said to be tame. If a plane graph $G(D)$ is associated with a contravening decomposition star $D$, we call $G(D)$ a contravening plane graph.

Theorem 4.4. Every contravening plane graph is tame.

This theorem is one of the main results of this paper. Its proof occupies Sections 5 through 9.

In Theorem 2.1, the tame graphs are classified up to isomorphism. As a corollary, we have an explicit list containing all contravening plane graphs.

\section{Contravention is tame}

This section begins the proof of Theorem 4.4.

To prove Theorem 4.4, it is enough to show that each defining property of tameness is satisfied. This is the substance of results in the next sections. First, we prove the promised non-degeneracy result.

Proposition 5.1. The construction of Section 4.1 associates a (nonempty) plane graph with at least two faces to every decomposition star $D$ with $\sigma(D)>0$.
Proof. First we show that decomposition stars with $\sigma(D)>0$ have non-empty vertex sets $U$. (Recall that $U$ is the set of vertices of distance at most $2 t_{0}$ from the center). The vertices of $U$ are used in [2] to create all of the structural features of the decomposition star: quasi-regular tetrahedra, quarters, and so forth. If $U$ is empty, the decomposition star is a solid containing the ball $B\left(t_{0}\right)$ of radius $t_{0}$, and its score is just

$$
\begin{aligned}
\sigma(D) & =\operatorname{vor}(D) \\
& =-4 \delta_{o c t} \operatorname{vol}(D)+4 \pi / 3 \\
& <-4 \delta_{o c t} \operatorname{vol}\left(B\left(t_{0}\right)\right)+4 \pi / 3 \quad<0 .
\end{aligned}
$$

By hypothesis, $\sigma(D)>0$. So $U$ is not empty.

The results of [2, Eqn 3.11] show that the function $\sigma$ can be expressed as a sum of terms $\sigma_{R}$ indexed by the standard regions $R$. It is proved in [2, Lemma 3.13 ] that $\sigma_{R} \leq 0$, unless $R$ is a triangle. Thus, a decomposition star with positive score must have at least one triangle. Its complement contains a second standard region. Even after we form aggregates of distinct standard regions to form the simplified plane graph (Remarks 4.2 and 4.3), there certainly remain at least two faces.

Proposition 5.2. The plane graph of a contravening decomposition star satisfies Property 1 of tameness: The length of each face is (at least 3 and) at most 8.

Proof. By the construction of the graph, each face has at least three edges. The upper bound of 8 faces is [6, Corollary 4.4].

Proposition 5.3. The plane graph of a contravening decomposition star satisfies Property 2 of tameness: Every 3-circuit is a face or the opposite of a face.

Proof. The simplifications of the plane graph in Remarks 4.2 and 4.3 do not produce any new 3circuits. (See the accompanying figures.) The result is proved in [3, Lemma 3.7]. The proof is purely geometric: it expresses a geometric impossibility true of all decomposition stars.

Proposition 5.4. Contravening graphs satisfy Property 4 of tameness: The degree of every vertex is at least 2 and at most 6.

Proof. The statement that degrees are at least 2 trivially follows because each vertex lies on at least one polygon, with two edges at that vertex.

The impossibility of a vertex of degree 7 or more is found in the proof of [5, Lemma 6.2]. 


\section{More Tame Properties}

This section continues in the proof that all contravening plane graphs are tame.

\subsection{Linear Programs}

To continue with the proof that contravening plane graphs are tame, we need to introduce some more notation and methods.

The function $\sigma$ can be written is a sum of functions on the space of decomposition stars, indexed by standard regions. (See [2, Eqn 3.11].) Write $\sigma_{R}$ for the term indexed by the standard region $R$. Then

$$
\sigma(D)=\sum_{R} \sigma_{R}(D)
$$

for every decomposition star.

Let $\zeta=1 /(2 \arctan (\sqrt{2} / 5)$. Let $\operatorname{sol}(R)$ denote the solid angle of a region $R$. We write $\tau_{R}$ for the following modification of $\sigma_{R}$ :

$$
\tau_{R}(D)=\operatorname{sol}(R) \zeta p t-\sigma_{R}(D)
$$

and

$$
\tau(D)=\sum \tau_{R}(D)=4 \pi \zeta p t-\sigma(D) .
$$

Since $4 \pi \zeta p t$ is a constant, $\tau$ and $\sigma$ contain the same information, but $\tau$ is often more convenient to work with. A contravening decomposition star satisfies

$$
\tau(D) \leq 4 \pi \zeta p t-8 p t=(4 \pi \zeta-8) p t .
$$

The constant $(4 \pi \zeta-8) p t$ (and its upper bound $14.8 p t$ ) will occur repeatedly in the discussion that follows.

Let $\mathcal{R}(F)$ be the set of standard regions associated with a face $F$ in the plane graph. We occasionally combine the terms $\sigma_{R}$ associated with the same face. The set contains a single standard region, unless multiple standard regions have been aggregated according to the constructions of Remarks 4.2 and 4.3. Set

$$
\sigma_{F}=\sum_{R \in \mathcal{R}(F)} \sigma_{R}
$$

and

$$
\tau_{F}=\sum_{R \in \mathcal{R}(F)} \tau_{R}
$$

In [6, Section 4.4] a natural number $n(R)$ is attached to the standard region $R$. Lower bounds on $\tau_{R}(D)$ are obtained in [6, Theorem 4.4] that depend only on $n(R)$. Write $t_{n}$ for the constants in this theorem. The same theorem gives upper bounds $s_{n}$ for $\sigma_{R}(D)$ that depend only on $n(R)$.

It is also helpful to bear in mind several bounds on angles in the following discussion. Every internal angle of every standard region is at least 0.8638 [5, 10.1.3]. Every internal angle of every standard region, except for triangles, is at least 1.153 [5, 4.3].

A decomposition star $D$ determines a set of vertices $U(D)$ that are of distance at most $2 t_{0}$ from the center of $D$. Three consecutive vertices $p_{1}, p_{2}$, and $p_{3}$ of a standard region are determined as the projections to the unit sphere of three corners $v_{1}$, $v_{2}$, and $v_{3}$, respectively in $U(D)$. If the internal angle of a standard region is less than 1.32, then it follows by an interval arithmetic calculation that $\left|v_{1}-v_{3}\right| \leq \sqrt{8}$, see [6, Lemma 3.11.4].

We are to the point in the proof of the Kepler Conjecture where it becomes necessary to use inequalities proved by interval arithmetic in a serious way. To use these inequalities systematically, we combine inequalities into linear programs and solve the linear programs on computer. At first, our use of linear programs will be light, but our reliance will become progressively strong as this paper develops.

To start out, we will make use of the inequalities $[5,10.3 .4-8]$ and $[5,4.1]$. These inequalities give lower bounds on $\tau_{R}(D)$ when $R$ is a triangle or quadrilateral. To obtain lower bounds through linear programming, we take a linear relaxation. Specifically, we introduce a linear variable for each function $\tau_{R}$ and a linear variable for each internal angle $\alpha_{R}$. We substitute these linear variables for the nonlinear functions $\tau_{R}(D)$ and nonlinear internal angle function into the given inequalities in [5]. Under these substitutions, the inequalities become linear. Given $p$ triangles and $q$ quadrilaterals at a vertex, we have the linear program to minimize the sum of the (linear variables associated with) $\tau_{R}(D)$ subject to the constraint that the (linear variables associated with the) angles at the vertex sum to at most $d$. Linear programming yields a lower bound $\tau_{\mathrm{LP}}(p, q, d)$ to this minimization problem. This gives a lower bound to the corresponding constrained sum of nonlinear functions $\tau_{R}$.

Similarly, the inequalities from the same sections of [6] yield upper bounds $\sigma_{\mathrm{LP}}(p, q, d)$ on the sum of $p+q$ functions $\sigma_{R}$, with $p$ standard regions $R$ that are triangular, and another $q$ that are quadrilateral. These linear programs find their first application in the proof of the following proposition.

Proposition 6.1. The plane graph of a contraven- 
ing decomposition star satisfies Property 6 of tameness:

$$
\sum_{F} c(\operatorname{len}(F)) \geq 8
$$

Proof. We will show that

$$
c(l e n(F)) p t \geq \sigma_{F}(D)
$$

Assuming this, the result follows for contravening stars $D$ :

$$
\begin{aligned}
\sum_{F} c(l e n(F)) p t & \geq \sum_{F} \sigma_{F}(D) \\
& =\sigma(D) \geq 8 p t
\end{aligned}
$$

We consider three cases for Inequality 4 . In the first case, assume that the face $F$ corresponds to exactly one standard region in the decomposition star. In this case, Inequality 4 follows directly from the bounds [6, Theorem 4.4].

In the second case, assume we are in the context of a pentagon $F$ formed in Remark 4.2. Then, again by $[6,4.4]$, we have

$$
\sigma_{F}(D) \leq(c(3)+c(8)) p t \leq c(5) p t .
$$

(Just examine the constants $c(k)$.)

In the third case, we consider the situation of Remark 4.3. The six standard regions score at most $s_{5}+\sigma_{\mathrm{LP}}(5,0,2 \pi-1.153)<c(8) p t$.

Proposition 6.2. Let $F$ be a face of a contravening plane graph $G(D)$. Then

$$
\tau_{F}(D) \geq d(\operatorname{len}(F)) p t
$$

Proof. Similar.

Lemma 6.3. Consider the aggregate in Remark 4.3.

1. There are at most two vertices (of a standard region) of degree six. If there are two, then they are adjacent vertices on a pentagon (Figure 11).

2. If a vertex of a pentagonal standard region has degree six, then the aggregate $F$ of the six faces satisfies

$$
\begin{aligned}
& \sigma_{F}(D)<s_{8}, \\
& \tau_{F}(D)>t_{8} .
\end{aligned}
$$

Proof. We begin with the second part of the lemma. The sum $\tau_{F}(D)$ over these six standard regions is at least

$$
t_{5}+\tau_{\mathrm{LP}}(5,0,2 \pi-1.153)>t_{8}
$$

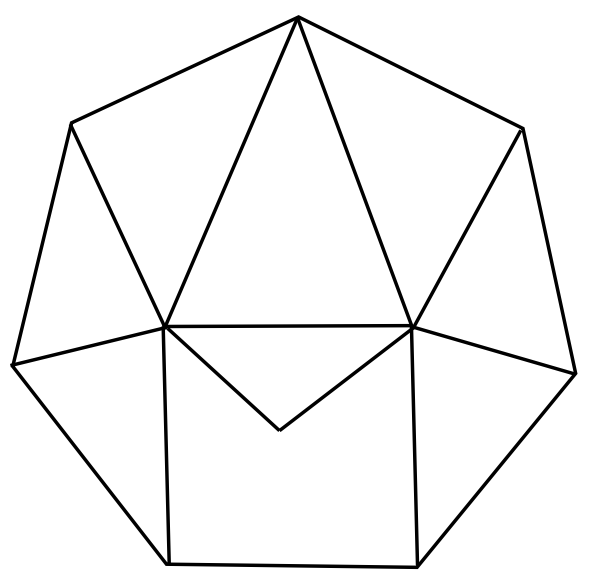

Figure 11: Adjacent vertices of degree 6

Similarly,

$$
s_{5}+\sigma_{\mathrm{LP}}(5,0,2 \pi-1.153)<s_{8} .
$$

We note that there can be at most one exceptional region with a vertex of degree six. Indeed, if there are two, then they must both be vertices of the same pentagon:

$$
t_{8}+t_{5}>(4 \pi \zeta-8) p t .
$$

Such a second vertex on the octagonal aggregate leads to one of the follow constants greater than $(4 \pi \zeta-8) p t$. These same constants show that such a second vertex on a hexagonal aggregate must share two triangular faces with the first vertex of degree six.

$$
\begin{array}{ll}
t_{8} & +\tau_{\mathrm{LP}}(4,0,2 \pi-1.32-0.8638), \quad \text { or } \\
t_{8} & +1.47 p t+\tau_{\mathrm{LP}}(4,0,2 \pi-1.153-0.8638), \\
t_{8} & +\tau_{\mathrm{LP}}(5,0,2 \pi-1.153) .
\end{array}
$$

(The relevant inequalities involving the constants 1.47 and 1.32 are found at [6, Lemma 5.12.1].)

Proposition 6.4. A contravening plane graph satisfies Property 5 of tameness: If a vertex is contained in an exceptional face, then the degree of the vertex is at most 5 .

Proof. Assume for a contradiction that $v$ is a vertex of degree six that lies in an exceptional region. There are several cases according to the number $k$ of triangular regions at the vertex.

$(k \leq 2)$ If there are at least four nontriangular regions at the vertex, then the sum of interior angles around the vertex is at least $4(1.153)+2(0.8638)>$ $2 \pi$, which is impossible. 
$(k=3)$ If there are three nontriangular regions at the vertex, then $\tau(D)$ is at least $2 t_{4}+t_{5}+$ $\tau_{\mathrm{LP}}(3,0,2 \pi-3(1.153))>(4 \pi \zeta-8) p t$.

$(k=4)$ If there are two exceptional regions at the vertex, then $\tau(D)$ is at least $2 t_{5}+\tau_{\mathrm{LP}}(4,0,2 \pi-$ $2(1.153))>(4 \pi \zeta-8) p t$.

If there are two nontriangular regions at the vertex, then $\tau(D)$ is at least $t_{5}+\tau_{\mathrm{LP}}(4,1,2 \pi-1.153)>$ $(4 \pi \zeta-8) p t$.

We are left with the case of five triangular regions and one exceptional region.

When there is an exceptional standard region at a vertex of degree six, we claim that the exceptional region must be a pentagon. If the region is a heptagon or more, then $\tau(D)$ is at least $t_{7}+\tau_{\mathrm{LP}}(5,0,2 \pi-1.153)>(4 \pi \zeta-8) p t$.

If the standard region is a hexagon, then $\tau(D)$ is at least $t_{6}+\tau_{\mathrm{LP}}(5,0,2 \pi-1.153)>t_{9}$. Also, $s_{6}+\sigma_{\mathrm{LP}}(5,0,2 \pi-1.153)<s_{9}$. The aggregate of the six standard regions is a nonagon. The argument of [6, Section 4.6] extends to this context to give the bound of $8 p t$.

\section{Weight Assignments}

The purpose of this section is to prove the existence of a good admissible weight assignment for contravening plane graphs.

Theorem 7.1. Every contravening planar graph has an admissible weight assignment of total weight less than 14.8 .

Given a contravening decomposition star $D$, we define a weight assignment $w(F)$ by

$$
w(F)=\tau_{F}(D) / p t
$$

Since $D$ contravenes,

$$
\begin{aligned}
\sum_{F} w(F) & =\sum_{F} \tau_{F}(D) / p t \\
& =\tau(D) \leq(4 \pi \zeta-8) p t / p t \\
& <14.8
\end{aligned}
$$

The challenge of the theorem will be to prove that $w(F)$, when defined by this formula, is admissible.

The next three lemmas establish that this definition of $w(F)$ for contravening plane graphs satisfies the first three defining properties of an admissible weight assignment.
Lemma 7.2. Let $F$ be a face of length $n$ in a contravening plane graph. Define $w(F)$ as above. Then $w(F) \geq d(n)$.

Proof. This is Proposition 6.2.

Lemma 7.3. Let $v$ be a vertex of type $(p, q)$ in a contravening plane graph. Define $w(F)$ as above. Then

$$
\sum_{v \in F} w(F) \geq b(p, q)
$$

Proof. This is [5, Proposition 5.2].

Lemma 7.4. Let $V$ be any set of vertices of type $(5,0)$ in a contravening plane graph. Define $w(F)$ as above. If the cardinality of $V$ is $k \leq 4$, then

$$
\sum_{V \cap F \neq \emptyset} w(F) \geq 0.55 k
$$

Proof. This is [5, Proposition 5.3].

The following proposition establishes the final property that $w(F)$ must satisfy to make it admissible.

Proposition 7.5. Let $V$ be any separated set of vertices in a contravening plane graph. Define $w(F)$ as above. Then

$$
\sum_{V \cap F \neq \emptyset}(w(F)-d(\operatorname{len}(F))) \geq \sum_{v \in V} a(\operatorname{tri}(v)),
$$

where tri $(v)$ denotes the number of triangles containing the vertex $v$.

The proof will occupy the rest of this section, as well as all of Section 8. Since the degree of each vertex is five, and there is at least one face that is not a triangle at the vertex, the only constants $\operatorname{tri}(v)$ that arise are

$$
\operatorname{tri}(v) \in\{0, \ldots, 4\}
$$

We will prove that in a contravening plane graph that the Properties (1) and (4) of a separated set are incompatible with the condition $\operatorname{tr} i(v) \leq 2$, for some $v \in V$.

This will allows us to assume that

$$
\operatorname{tri}(v) \in\{3,4\}
$$

for all $v \in V$. These cases will be treated in Sections 7.3 and 7.4 . 
The outline of the proof of Proposition 7.5 is the following. Let $D$ be a contravening decomposition star. First, assume that every face meeting $V$ corresponds to a single standard region of $D$. (That is, we exclude the aggregates created in Remark 4.2 and Remark 4.3.) Under this hypothesis, we show that $\operatorname{tri}(v) \leq 2$ is incompatible with the hypothesis. This allows us to assume that $\operatorname{tri}(v) \in\{3,4\}$. We express the desired inequality as a sum of inequalities indexed by $V$, where the inequality indexed by $v \in V$ depends only on (an explicit description of) the function $\tau_{R}(D)$ for standard regions $R$ corresponding to a face containing $v$. These individual inequalities are proved first for $\operatorname{tri}(v)=3$, and then for $\operatorname{tri}(v)=4$.

Finally in Section 8, we return to the aggregates of Remark 4.2 and Remark 4.3 and show that the conclusions can be extended to aggregate faces as well.

\subsection{Proof that $\operatorname{tri}(v)>2$}

In this subsection $D$ is a contravening decomposition star with associated graph $G(D)$. Let $V$ be a set of vertices that satisfies the conditions of Proposition 7.5. Let $v$ be a vertex in $V$ such that none of its faces is an aggregate in the sense of Remarks 4.2 and 4.3 .

Lemma 7.6. Under these conditions, for every $v \in$ $V, \operatorname{tri}(v)>1$.

Proof. If there are $p$ triangles, $q$ quadrilaterals, and $r$ other faces, then

$$
\begin{aligned}
\tau(D) & \geq \sum_{v \in R} \tau_{R}(D) \\
& \geq r t_{5}+\tau_{\mathrm{LP}}(p, q, 2 \pi-r(1.153)) .
\end{aligned}
$$

If there is a vertex $w$ that is not on any of the faces containing $v$, then the sum of $\tau_{F}(D)$ over the faces containing $w$ yield an additional $0.55 p t$ by $[5,5.2]$. We run the linear programs for each $(p, q, r)$ and find that the bound is always greater than $(4 \pi \zeta-$ 8) pt. This implies that $D$ cannot be contravening.

$$
\begin{array}{cc}
(p, q, r) & \text { lower bound } \\
(0,5,0) & 22.27 p t[5,5.2] \\
(0, q, r \geq 1) & t_{5}+4 t_{4} \approx 14.41 p t \\
(1,4,0) & 17.62 p t[5,5.2] \\
(1,3,1) & t_{5}+12.58 p t\left(\tau_{\mathrm{LP}}\right) \\
(1,2,2) & 2 t_{5}+7.53 p t\left(\tau_{\mathrm{LP}}\right) \\
(1, q, r \geq 3) & 3 t_{5}+t_{4}
\end{array}
$$

Lemma 7.7. Under these same conditions, for every $v \in V, \operatorname{tri}(v)>2$.

Proof. Assume that $\operatorname{tri}(v)=2$. We will show that this implies that $D$ is not contravening. Let $e$ be the number of exceptional faces at $v$.

The constants $0.55 p t$ and $4.52 p t$ used throughout the proof come from $[5,5.3]$; The constants $t_{n}$ comes from $[6,4.4]$.

$(e=3)$ : First, assume that there are three exceptional faces around vertex $v$. They must be pentagons $\left(2 t_{5}+t_{6}>(4 \pi \zeta-8) p t\right)$. The aggregate of the five faces is an $m$-gon (some $m \leq 11$ ). If there is a vertex not on this aggregate, use $3 t_{5}+0.55 p t>(4 \pi \zeta-8) p t$. So there are at most nine triangles away from the aggregate.

$$
\sigma(D) \leq 9 p t+\left(3 s_{5}+2 p t\right)<8 p t
$$

The argument if there is a quad, pentagon, and hexagon is the same $\left(t_{4}+t_{6}=2 t_{5}, s_{4}+s_{6}=2 s_{5}\right)$.

$(e=2)$ : Assume next that there are two pentagons and a quadrilateral around the vertex. The aggregate of the two pentagons, quadrilateral, and two triangles is an $m$-gon (some $m \leq 10$ ). There must be a vertex not on the aggregate of five faces, for otherwise we have

$$
\sigma(D) \leq 8 p t+\left(2 s_{5}+2 p t\right)<8 p t .
$$

The interior angle of one of the pentagons is at most 1.32. For otherwise, $\tau_{\mathrm{LP}}(2,1,2 \pi-2(1.32))+$ $2 t_{5}+0.55 p t>(4 \pi \zeta-8) p t$.

The calculation $[6$, Lemma 5.12.2] shows that any pentagon $R$ with an internal angle less than 1.32 yields $\tau_{R}(D) \geq 5.66 p t$. If both pentagons have an interior angle $<1.32$ the lemma follows easily from this calculation: $2(5.66) p t+\tau_{\mathrm{LP}}(2,1,2 \pi-$ $2(1.153))+0.55 p t>(4 \pi \zeta-8) p t$. If there is one pentagon with angle $>1.32$, we then have $5.66 p t+\tau_{\mathrm{LP}}(2,1,2 \pi-1.153-1.32)+t_{5}+0.55 p t>$ $(4 \pi \zeta-8) p t$.

$(e=1)$ : Assume finally that there is one exceptional face at the vertex. If it is a hexagon (or more), we are done $t_{6}+\tau_{\mathrm{LP}}(2,2,2 \pi-1.153)>$ $(4 \pi \zeta-8) p t$. Assume it is a pentagon. The aggregate of the five faces at the vertex is bounded by an $m$-circuit (some $m \leq 9$ ). If there are no more than 11 quasi-regular tetrahedra outside the aggregate, the score is at most $(1+2(4.52)) p t+s_{5}+$ $\sigma_{\mathrm{LP}}(2,2,2 \pi-1.153)<8 p t([5,5.3])$. So we may assume that there are at least three vertices not on the aggregate. 
If the interior angle of the pentagon is greater than 1.32 , we have

$\tau_{\mathrm{LP}}(2,2,2 \pi-1.32)+3(0.55) p t+t_{5}>(4 \pi \zeta-8) p t$

and if it is less than 1.32 , we have by [6, Lemma $5.12 .1]$

$$
\begin{aligned}
\tau_{\mathrm{LP}}(2,2,2 \pi-1.153) & +3(0.55) p t+1.47 p t+t_{5} \\
& >(4 \pi \zeta-8) p t
\end{aligned}
$$

Lemma 7.8. The bound $\operatorname{tri}(v)>2$ holds if $v$ is a vertex of an aggregate face.

Proof. The exceptional region enters into the preceding two proofs in a purely formal way. Pentagons enter through the bounds

$$
t_{5}, s_{5}, 1.47 p t, 5.66 p t
$$

and angles 1.153, 1.32. Hexagons enter through the bounds

$$
t_{6}, s_{6}
$$

and so forth. These bounds hold for the aggregate faces. Hence the proofs hold for aggregates as well.

\subsection{Bounds when $\operatorname{tri}(v) \in\{3,4\}$.}

In this subsection $D$ is a contravening decomposition star with associated graph $G(D)$. Let $V$ be a separated set of vertices. For every vertex $v$ in $V$, we assume that none of its faces is an aggregate in the sense of Remarks 4.2 and 4.3. We assume that there are three or four triangles containing $v$, for every $v \in V$.

To prove the Inequality 4 in the definition of admissible weight assignments, we will rely on the following reductions. Define an equivalence relation on exceptional faces by $F \sim F^{\prime}$ if there is a sequence $F_{0}=F, \ldots, F_{r}=F^{\prime}$ of exceptional faces such that consecutive faces share a vertex of type $(3,0,2)$. Let $\mathcal{F}$ be an equivalence class of faces.

Lemma 7.9. Let $V$ be a separated set of vertices. For every equivalence class of exceptional faces $\mathcal{F}$, let $V(\mathcal{F})$ be the subset of $V$ whose vertices lie in the union of faces of $\mathcal{F}$. Suppose that for every equivalence class $\mathcal{F}$, the Inequality 4 (in the definition of admissible weight assignments) holds for $V(\mathcal{F})$. Then the Inequality holds for $V$.
Proof. By construction, each vertex in $V$ lies in some $F$, for an exceptional face. Moreover, the separating property of $V$ insures that the triangles and quadrilaterals in the inequality are associated with a well-defined $\mathcal{F}$. Thus, the inequality for $V$ is a sum of the inequalities for each $V(\mathcal{F})$.

Lemma 7.10. Let $v$ be a vertex at which there are p triangles, q quadrilaterals, and $r$ other faces. Suppose that for some $p^{\prime} \leq p$ and $q^{\prime} \leq q$, we have

$$
\tau_{\mathrm{LP}}\left(p^{\prime}, q^{\prime}, \alpha\right)>\left(p^{\prime} d(3)+q^{\prime} d(4)+a(p)\right) p t
$$

for some upper bound $\alpha$ on the angle occupied by $p^{\prime}$ triangles and $q^{\prime}$ quadrilaterals at $v$. Suppose further that the Inequality 4 (in the definition of admissible weight assignments) holds for $V^{\prime}=V \backslash\{v\}$. Then the inequality holds for $V$.

Proof. Let $F_{1}, \ldots, F_{m}, m=p^{\prime}+q^{\prime}$, be faces corresponding to the triangles and quadrilaterals in the lemma. The hypotheses of the lemma imply that

$$
\sum_{1}^{m}\left(\tau_{F_{i}}(D)-d\left(\operatorname{len}\left(F_{i}\right)\right)\right)>a(p) .
$$

Clearly, the Inequality for $V$ is the sum of this inequality, the inequality for $V^{\prime}$, and $d(n) \geq 0$.

\section{3 $\operatorname{tri}(v)=3$}

To continue further, we need to recall from [6] how $\tau_{R}(D)$, for exceptional standard regions, can be written as a sum of terms in a way that reflects the internal geometry of $D$ over $R$. We have prepared a summary of the relevant material from [6] in Appendix 16. We recommend that the reader review Appendix 16 before reading the next lemma. We use terminology from that appendix (penalties, loops, erasure, flat quarters, $\left.D(n, k), \delta_{\text {loop }}(n, k), \hat{\sigma}\right)$.

Lemma 7.11. Property 4 of admissibility holds when $\operatorname{tri}(v)=3$ for all $v \in V$.

Proof. When there are three triangles at a vertex of degree five along with an exceptional face, the relevant constant from the definition of admissibility is $a(3)=1.4$. Erase all upright quarters except those in loops. The result follows from [6, Lemma 5.12.1]. and the subsequent remark if the internal angle at the vertex of an exceptional region is $\leq 1.32$.

Assume the angles on the exceptional regions are $\geq 1.32$. If there are three triangles, a quad, and an exceptional face at the vertex, then we have $\tau_{\mathrm{LP}}(3,1,2 \pi-1.32)>1.4 p t+t_{4}$. 
The final case is three triangles and two exceptional faces at the vertex. There can be no heptagons and at most one hexagon $2 t_{6}=t_{5}+t_{7}>$ $(4 \pi \zeta-8) p t$. In these calculations, the flat quarters are scored by $\hat{\sigma}$. By Appendix 16, each flat quarter contributes $D(3,1)$ to $t_{n}$. The constant $a(\operatorname{tri}(v))$ pt estimates what the flat quarter and surrounding quasi-regular tetrahedra squander in excess of $D(3,1)$. Most of the calculations now follow directly from the interval calculations of Appendix 17.20. A few additional comments are needed if there is a loop masking a flat quarter. If the constant $\delta_{\text {loop }}(n, k)$ exceeds $r(1.4) p t$, where $r$ is the greatest possible number of masked flat quarters within the loop, then the result follows. This gives every case except $(n, k)=(4,1),(5,0)$. If $(n, k)=$ $(4,1)$, we erase and take the penalty $\pi_{F}=2 \xi_{V}+\xi_{\Gamma}$ whenever the internal angle is at least 1.32. This penalty appears in Appendix 17.20.

If $(n, k)=(5,0)$, there are various possibilities. If there is one masked flat quarter, use $\delta_{\text {loop }}(5,0)>$ $1.4 \mathrm{pt}$. If there are two masked flat quarters, erase with penalty $\pi_{F}=2 \xi_{V}+\xi_{\Gamma} / 2$ at each flat quarter. If the internal angle is at most 1.32, use

$$
3.07 p t-D(3,1)>1.4 p t+\pi_{F} .
$$

If the internal angle is at least 1.32, use the interval calculations Appendix 17.20 with penalties.

\section{$7.4 \operatorname{tri}(v)=4$}

If there are four triangles and one exceptional region at a vertex, we justify the constant $a(\operatorname{tr} i(4))=$ $1.5 \mathrm{pt}$, in the definition of admissible weight assignment. These calculations are similar to those of Section 7.3 and are based on the interval calculations Appendix 17.21. Details are left to the reader. It is simpler because there is only one exceptional region, and it is not necessary to separate the case according to the size of the internal angle (at least 1.32 and at most 1.32).

\section{The Aggregate Cases}

\subsection{The non-polygonal standard re- gions}

Lemma 8.1. Consider a separated set of vertices $V$ on an aggregated face $F$ as in Remark 4.2. The Inequality 4 holds (in the definition of admissible weight assignments):

$$
\sum_{V \cap F \neq \emptyset}(w(F)-d(\operatorname{len}(F))) \geq \sum_{v \in V} a(\operatorname{tri}(v)) .
$$

Proof. We may assume that $\operatorname{tri}(v) \in\{3,4\}$.

First consider the aggregate of Remark 4.2 of a triangle and eight-sided region, with pentagonal hull $F$. There is no other exceptional region in a contravening decomposition star with this aggregate:

$$
t_{8}+t_{5}>(4 \pi \zeta-8) p t
$$

A separated set of vertices $V$ on $F$ has cardinality at most 2. This gives the desired bound

$$
t_{8}>t_{5}+2(1.5) p t
$$

Next, consider the aggregate of a hexagonal hull with an enclosed vertex. Again, there is no other exceptional face. If there are at most $k \leq 2$ vertices in a separated set, then the result follows from

$$
t_{8}>t_{6}+k(1.5) p t
$$

There are at most three vertices in $V$ on a hexagon, by the non-adjacency conditions defining $V$. A vertex $v$ can be removed from $V$ if it does not lie on a flat quarter (Lemma 7.10 and Appendix 17.21). However, if there is an enclosed vertex $w$, it is impossible for there to be three nonadjacent vertices, none lying along a flat quarter:

$$
\mathcal{E}\left(2,2,2, \sqrt{8}, \sqrt{8}, \sqrt{8}, 2 t_{0}, 2 t_{0}, 2\right)>2 t_{0} .
$$

(The definition of $\mathcal{E}$ can be reviewed at $[2$, Section 1].)

Finally consider the aggregate of a pentagonal hull with an enclosed vertex. There are at most $k \leq 2$ vertices in a separated set in $F$. There is no other exceptional region:

$$
t_{7}+t_{5}>(4 \pi \zeta-8) p t
$$

The result follows from

$$
t_{7}>t_{5}+2(1.5) p t
$$

Lemma 8.2. Consider a separated set of vertices $V$ on an aggregate face of a contravening plane graph as in Remark 4.3. The Inequality 4 holds in the definition of admissible weight assignments. 
Proof. There is at most one exceptional face in the plane graph:

$$
t_{8}+t_{5}>(4 \pi \zeta-8) p t .
$$

Assume first that aggregate face is an octagon (Figure 10). At each of the vertices of the face that lies on a triangular standard region in the aggregate, we can remove the vertex from $V$ using Lemma 7.10 and the estimate

$$
\tau_{\mathrm{LP}}(4,0,2 \pi-2(0.8638))>1.5 p t .
$$

This leaves at most one vertex in $V$, and it lies on a vertex of $F$ which is "not aggregated," so that there are five standard regions of the underlying decomposition star at that vertex, and one of those regions is pentagonal. The value $a(4)=1.5 p t$ can be estimated at this vertex in the same way it is done for a non-aggregated case. (We use the calculations of Appendix 17.21.)

Now consider the case of an aggregate face that is a hexagon (Figure 10). The argument is the same: we reduce to $V$ containing a single vertex, and argue that this vertex can be treated by the inequalities of Appendix 17.21.

\section{$9 \quad$ Four-cycles}

It has now been verified that all the properties of tameness except one have been verified. This section verifies that contravening plane graphs satisfy this final property, and in this way we complete the proof that all contravening plane graphs are tame.

\subsection{Possible four-circuits}

Every 4-circuit divides a plane graph into two aggregates of faces that we may call the interior and exterior. We call vertices of the faces in the aggregate that do not lie on the 4-cycle enclosed vertices. Thus, every vertex lies in the 4-cycle, is enclosed over the interior, or is enclosed over the exterior.

Proposition $[3,4.2]$ asserts that either the interior or the exterior has at most 1 enclosed vertex. (The proof does not require the hypothesis that the graph be contravening. It expresses a geometric constraint holding for all decomposition stars.) When choosing which aggregate is to be called the interior, we may make our choice so that the interior contains at most 1 enclosed vertex. With this choice, we have the following proposition.
Proposition 9.1. Let $D$ be a contravening plane graph. The interior of a 4-circuit surrounds is one of the aggregates of faces shown in Property 3 of tameness.

Proof. If there are no enclosed vertices, then the only possibilities are for it to be a single quadrilateral face or a pair of adjacent triangles.

Assume there is one enclosed vertex $v$. If $v$ is connected to 3 or 4 vertices of the quadrilateral, then that possibility is listed as part of the conclusion.

If $v$ is connected to 2 opposite vertices in the 4-cycle, then the vertex $v$ has type $(0,2)$ and the bounds of [5, Proposition 5.2] show that the graph cannot be contravening.

If $v$ is connected to 2 adjacent vertices in the 4cycle, then we appeal to Lemma 9.3 to conclude that the graph is not contravening.

If $v$ is connected to 0 or 1 vertices, then we appeal to [5, Lemma 2.2], which shows that this creates an impossible geometric situation. This completes the proof.

\subsection{A non-contravening Four-circuit}

This subsection rules out the existence of a particular four-circuit on a contravening plane graph. The interior of the circuit consists of two faces: a triangle and a pentagon. The circuit and its enclosed vertex are show in Figure 12 with vertices marked $p_{1}, \ldots, p_{5}$. The vertex $p_{1}$ is the enclosed vertex, the triangle is $\left(p_{1}, p_{2}, p_{5}\right)$ and the pentagon is $\left(p_{1}, \ldots, p_{5}\right)$.

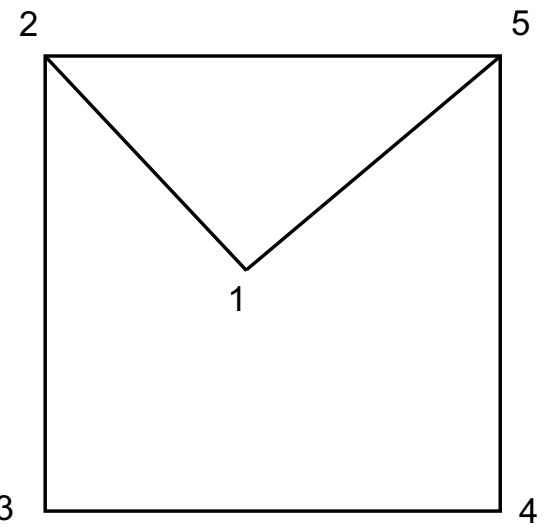

Figure 12: A non-contravening 4-circuit

Suppose that $D$ is a decomposition star whose associate graph contains such a 4-circuit. Recall that 
$D$ determines a set $U(D)$ of vertices in Euclidean 3 -space of distance at most $2 t_{0}$ from the center (the origin) of $D$, and that each vertex $p_{i}$ can be realized geometrically as a point on the unit sphere at the origin, obtained as the radial projection of some $v_{i} \in U(D)$.

In the rest of this subsection, we assume that the reader is familiar with techniques in [6]. (In particular, the reader will need to be familiar with geometric considerations and the details about how the function $\tau_{R}(D)$ is estimated for a pentagonal standard region $R$. This is reviewed in Appendix 16.)

Lemma 9.2. One of the edges $\left(v_{1}, v_{3}\right),\left(v_{1}, v_{4}\right)$ has length less than $2 \sqrt{2}$. Both of the them have lengths less than 3.02. Also, $\left|v_{1}\right| \geq 2.3$.

Proof. This is a standard exercise in geometric considerations ([2, Section 1] and [5, Section 2]). (The reader should review these sections for the framework of the following argument.) We deform the figure using pivots to a configuration $v_{2}, \ldots, v_{5}$ at height 2 , and $\left|v_{i}-v_{j}\right|=2 t_{0},(i, j)=$ $(2,3),(3,4),(4,5),(5,2)$. We scale $v_{1}$ until $\left|v_{1}\right|=$ $2 t_{0}$. We can also take the distance from $v_{1}$ to $v_{5}$ and to $v_{2}$ to be 2 . If we have $\left|v_{1}-v_{3}\right| \geq 2 \sqrt{2}$, then we stretch the edge $\left|v_{1}-v_{4}\right|$ until $\left|v_{1}-v_{3}\right|=2 \sqrt{2}$. The resulting configuration is rigid. Pick coordinates to find that $\left|v_{1}-v_{4}\right|<2 \sqrt{2}$. If we have $\left|v_{1}-v_{3}\right| \geq 2 t_{0}$, follow a similar procedure to reduce to the rigid configuration $\left|v_{1}-v_{3}\right|=2 t_{0}$, to find that $\left|v_{1}-v_{4}\right|<3.02$. The estimate $\left|v_{1}\right| \geq 2.3$ is similar.

There are restrictive bounds on the dihedral angles of the simplices $\left(0, v_{1}, v_{i}, v_{j}\right)$ along the edge $\left(0, v_{1}\right)$. The quasi-regular tetrahedron has a dihedral angle of at most 1.875 ([5, 10.1.2]). The dihedral angles of the simplices $\left(0, v_{1}, v_{2}, v_{3}\right)$, $\left(0, v_{1}, v_{5}, v_{4}\right)$ adjacent to it are at most 1.63. The dihedral angle of the remaining simplex $\left(0, v_{1}, v_{3}, v_{4}\right)$ is at most 1.51. (These constants come from 1.624 and 1.507 in Appendix 17.8.) This leads to lower bounds as well. The quasi-regular tetrahedron has a dihedral angle that is at least $2 \pi-2(1.63)-1.51>$ 1.51. The dihedral angles adjacent to the quasiregular tetrahedron is at least $2 \pi-1.63-1.51-$ $1.875>1.26$. The remaining dihedral angle is at least $2 \pi-1.875-2(1.63)>1.14$.

Lemma 9.3. A decomposition star that contains this configuration is not contravening: $\tau(D)>$ $(4 \pi \zeta-8) p t$.
Proof. We will show that the sum of $\tau_{R}(D)$, as $R$ ranges over the pentagonal standard region and triangular regions, is at least $11.16 p t$. Let $P$ denote the aggregate formed by these standard regions.

First we show how the lemma follows from this bound. There are no other exceptional faces, because $11.16 p t+t_{5}>(4 \pi \zeta-8) p t$. Every vertex not on $P$ has type $(5,0)$, by $[5$, Proposition 5.2]. In particular, there are no quadrilateral regions. There are at most 4 triangles at every vertex of $P$, because the $\tau_{\mathrm{LP}}(5,0,2 \pi-1.32)>6.02 p t$. (The 1.32 comes from that fact that $P$ has no flat quarters because $v$ is too short to be enclosed over one $[2,1.3]$ $[6,3.11 .4]$.) There are at least 3 triangles at every vertex of $P$, otherwise we contradict a property of tameness (Property 2 and Property 3 ).

The only triangulation with these properties is obtained by removing one edge from the icosahedron (Exercise). This implies that there are two opposite corners of $P$ each having four quasi-regular tetrahedra. Since the diagonals of $P$ have lengths greater than $2 \sqrt{2}$, the results of Section 7.4 show that these eight quasi-regular tetrahedra squander at least 2(1.5) pt. There are two additional vertices of type $(5,0)$ whose tetrahedra are distinct from these eight quasi-regular tetrahedra. They give an additional $2(0.55) \mathrm{pt}$. Now $(11.16+2(1.5)+$ $2(0.55)) p t>(4 \pi \zeta-8) p t$. The result follows.

Return to the bound $11.16 p t$. (A review of Appendix 16 is recommended before continuing. The definitions of $\tau, \hat{\tau}$ and $\tau_{0}$ are reviewed in Appendix 15.) Let dih denote the dihedral angle of a simplex along a given edge. Let $S_{i j}$ be the simplex $\left(0, v_{1}, v_{i}, v_{j}\right)$, for $(i, j)=(2,3),(3,4),(4,5),(2,5)$. We have $\sum_{(4)} \operatorname{dih}\left(S_{i j}\right)=2 \pi$. Suppose one of the edges $\left(v_{1}, v_{3}\right)$ or $\left(v_{1}, v_{4}\right)$ has length $\geq 2 \sqrt{2}$. Say $\left(v_{1}, v_{3}\right)$.

We have (by Appendix 17.1)

$$
\begin{array}{ll}
\tau\left(S_{25}\right) & -0.2529 \operatorname{dih}\left(S_{25}\right)>-0.3442 \\
\tau_{0}\left(S_{23}\right) & -0.2529 \operatorname{dih}\left(S_{23}\right)>-0.1787 \\
\hat{\tau}\left(S_{45}\right) & -0.2529 \operatorname{dih}\left(S_{45}\right)>-0.2137 \\
\tau_{0}\left(S_{34}\right) & -0.2529 \operatorname{dih}\left(S_{34}\right)>-0.1371
\end{array}
$$

We have by $\left[6, \mathbf{A}_{10}\right]$ that

$$
\begin{aligned}
\tau(D) \geq & \sum_{(4)} \tau_{x}\left(S_{i j}\right)-5 \xi_{\Gamma} \\
> & 2 \pi(0.2529)-0.3442 \\
& \quad-0.1787-0.2137-0.1371-5 \xi_{\Gamma} \\
& >11.16 p t
\end{aligned}
$$

where $\tau_{x}=\tau, \hat{\tau}, \tau_{0}$ as appropriate.

Now suppose $\left(v_{1}, v_{3}\right)$ and $\left(v_{1}, v_{4}\right)$ have length $\leq$ $2 \sqrt{2}$. If there is an upright diagonal that is not 
enclosed over either flat quarter, the penalty is at most $3 \xi_{\Gamma}+2 \xi_{V}$. Otherwise, the penalty is smaller: $4 \xi_{\Gamma}^{\prime}+\xi_{V}$. We have

$$
\begin{aligned}
\tau(D) \geq & \sum_{(4)} \tau\left(S_{i j}\right)-\left(3 \xi_{\Gamma}+2 \xi_{V}\right) \\
> & 2 \pi(0.2529)-0.3442 \\
& \quad-2(0.2137)-0.1371-\left(3 \xi_{\Gamma}+2 \xi_{V}\right) \\
& >11.16 p t .
\end{aligned}
$$

The proof that contravening plane graphs are tame is complete.

\section{Linear Programs}

We have completed the first half of the proof of the Kepler Conjecture by proving that every contravening plane graph is tame.

The second half of the proof of the Kepler Conjecture consists in showing that tame graphs are not contravening, except for the isomorphism class of graphs isomorphic to $G_{f c c}$ and $G_{h c p}$ associated with the face-centered cubic and hexagonal close packings.

The paper [5] treats all contravening tame graphs in which every face has length three or four, except for three cases $G_{f c c}, G_{p e n t}$, and $G_{h c p}$. The two cases $G_{f c c}$ and $G_{h c p}$ are treated in [2,3.13], and the case $G_{\text {pent }}$ is treated in [7].

We complete the proof of the Kepler Conjecture by analyzing all contravening plane graphs that contain at least one exceptional face. The primary tool that will be used is linear programming. The linear programs are obtained as relaxations of the original nonlinear optimization problem of maximizing $\sigma(D)$ over all decomposition stars whose associated graph is a given tame graph $G$. The upper bounds obtained through relaxation are upper bounds to the nonlinear problem.

To eliminate a tame graph, we must show that it is not contravening. By definition, this means we must show that $\sigma(D)<8 p t$. When a single linear program does not yield an upper bound under $8 \mathrm{pt}$, we branch into a sequence of linear programs that collectively imply the upper bound of $8 p t$. This will call for a sequence of increasingly complex linear programs.

\subsection{Relaxation}

(NLP) Let $f: P \rightarrow \mathbb{R}$ be a function on a nonempty set $P$. Consider the nonlinear maximization problem

$$
\max _{p \in P} f(p)
$$

(LP): Consider a linear programming problem

$$
\max c \cdot x
$$

such that $A x \leq b$, where $A$ is a matrix, $b, c$ are vectors of real constants and $x$ is a vector of variables $x=\left(x_{1}, \ldots, x_{n}\right)$. We write the linear programming problem as

$$
\max (c \cdot x: A x \leq b) .
$$


An interpretation $I$ of a linear programming problem (LP) is a set $|I|$, together with an assignment $x_{i} \mapsto x_{i}^{I}$ of functions $x_{i}^{I}:|I| \rightarrow \mathbb{R}$ to variables $x_{i}$. We say the constraints $A x \leq b$ of the linear program are satisfied under the interpretation $I$ if for all $p \in|I|$, we have

$$
A x^{I}(p) \leq b
$$

The interpretation $I$ is said to be a relaxation of the nonlinear program (NLP), if the following three conditions hold.

1. $P=|I|$.

2. The constraints are satisfied under the interpretation.

3. $f(p) \leq c \cdot x^{I}(p)$, for all $p \in|I|$.

Lemma 10.1. Let (LP) be a linear program with relaxation $I$ to $(N L P)$. Then (LP) has a feasible solution. Moreover, if (LP) is bounded above by a constant $M$, then $M$ is an upper bound on the function $f:|I| \rightarrow \mathbb{R}$.

Proof. A feasible solution is $x_{i}=x_{i}^{I}(p)$, for any $p \in|I|$. The rest is clear.

Remark 10.2. In general, it is to be expected that the interpretations $A x^{I} \leq b$ will be nonlinear inequalities on the domain $P$. In our situation, satisfaction of the inequality will be proved by interval arithmetic. Thus, the construction of an upper bound to (NLP) breaks into two tasks: to solve the linear programs and to prove the nonlinear inequalities required by the interpretation.

There are many nonlinear inequalities that enter into our interpretation, which have been proved by interval arithmetic on computer. These inequalities are contained in Appendix 17.

Remark 10.3. There is a second method of establishing the satisfaction of inequalities under an interpretation. Suppose that we wish to show that the inequality $e \cdot x \leq b^{\prime}$ is satisfied under the interpretation $I$. Suppose that we have already established that a system of inequalities $A x \leq b$ is satisfied under the interpretation $I$. We solve the linear programming problem $\max (e \cdot x: A x \leq b)$. If this maximum is at most $b^{\prime}$, then the inequality $e \cdot x \leq b^{\prime}$ is satisfied under the interpretation $I$. We will refer to $e \cdot x \leq b^{\prime}$ as an $L P$-derived inequality (with respect to the system $A x \leq b$ ).

\subsection{The Linear Programs}

Let $G$ be a tame plane graph containing an exceptional face. Let $X_{G}$ be the space of all decomposition stars whose associated plane graph is isomorphic to $G$.

Theorem 10.4. For every tame plane graph $G$ containing at least one exceptional face, there exists a finite sequence of linear programs with the following properties.

1. Every linear program has an admissible solution and its solution is strictly less than 8 pt.

2. For every linear program in this sequence, there is an interpretation I of the linear program that is a relaxation of the nonlinear optimization problem

$$
\sigma:|I| \rightarrow \mathbb{R}
$$

where $|I|$ is a subset of $X_{G}$.

3. The union of the subsets $|I|$, as we run over the sequence of linear programs, is $X_{G}$.

The proof is constructive. For every tame plane graph $G$ a sequence of linear programs is generated by computer and solved. The optimal solutions are all bounded above by $8 p t$. It will be clear from construction of the sequence that the union of the sets $|I|$ exhausts $X_{G}$. We estimate that nearly $10^{5}$ linear programs are involved in the construction.

Remark 10.5. The paper [9, Section 3.1.1] shows how the linear programs that arise in connection with the Kepler Conjecture can be formulated in such a way that they always have a feasible solution and so that the optimal solution is bounded. We assume that all our linear programs have been constructed in this way.

Corollary 10.6. If a tame graph $G$ contains an exceptional face, then it is not contravening.

Proof. This follows immediately from the theorem and Lemma 10.1.

\section{LP Formulation}

\subsection{Basic Linear Programs}

To describe the basic linear program, we need the following indexing sets. 
Let VERTEX be the set of all vertices in $G$. Let FACE be the set of all faces in $G$. (Recall that by construction each face $F$ of the graph carries an orientation.) Let ANGLE be the set of all angles in $G$, defined as the set of pairs $(v, F)$, where the vertex $v$ lies in the face $F$. Let DIRECTED be the set of directed edges. It consists of all ordered pairs $(v, s(v, F))$, where $s(v, F)$ denotes the successor of the vertex $v$ in the oriented face $F$. Let TRIANGLES be the subset of FACE consisting of those faces of length 3. Let UNDIRECTED be the set of undirected edges. It consists of all unordered pairs $\{v, s(v, F)\}$, for $v \in F$.

We introduce variables indexed by these sets. Following AMPL notation, we write for instance $y\{$ VERTEX $\}$ to declare a collection of variables $y[v]$ indexed by vertices $v$ in VERTEX. With this in mind, we declare the variables

$$
\begin{array}{lll}
\alpha\{\text { ANGLE }\}, & y\{\text { VERTEX }\}, & e\{\text { UNDIRECTED }\}, \\
\sigma\{\text { FACE }\}, & \tau\{\text { FACE }\}, & \text { sol }\{\text { FACE }\} .
\end{array}
$$

We obtain an interpretation $I$ on the compact space $X_{G}$. First, we define an interpretation at the level of indexing sets. A decomposition star determines the set $U(D)$ of vertices of height at most $2 t_{0}$ from the origin of $D$. Each decomposition star $D \in X_{G}$ determines a (metric) graph with geodesic edges on the surface of the unit sphere, which is isomorphic to $G$ as a (combinatorial) plane graph. There is a map from the vertices of $G$ to $U(D)$ given by $v \mapsto v^{I}$, if the radial projection of $v^{I}$ to the unit sphere at the origin corresponds to $v$ under this isomorphism. Similarly, each face $F$ of $G$ corresponds to a set $F^{I}$ of standard regions. Each edge $e$ of $G$ corresponds to a geodesic edge $e^{I}$ on the unit sphere.

Now we give an interpretation $I$ to the linear programming variables at a decomposition star $D$. As usual, we add a superscript $I$ to a variable to indicate its interpretation. Let $\alpha[v, F]^{I}$ be the sum of the internal angles at $v^{I}$ of the metric graph in the standard regions $F^{I}$. Let $y[v]^{I}$ be the length $\left|v^{I}\right|$ of the vertex $v^{I} \in U(D)$ corresponding to $v$. Let $e[v, w]^{I}$ be the length $\left|v^{I}-w^{I}\right|$ of the edge between $v^{I}$ and $w^{I} \in U(D)$. Let

$$
\begin{array}{ll}
\sigma[F]^{I} & =\sigma_{F}(D), \\
\operatorname{sol}[F]^{I} & =\operatorname{sol}\left(F^{I}\right), \\
\tau[F]^{I} & =\tau_{F}(D) .
\end{array}
$$

The objective function for the optimization problems is

$$
\max : \sum_{F \in \mathrm{FACE}} \sigma_{F} \text {. }
$$

Its interpretation under $I$ is the score $\sigma(D)$.

We can write a number of linear inequalities that will be satisfied under our interpretation. For example, we have the bounds

$$
\begin{array}{ll}
0 \leq y[v] \leq 2 t_{0}, & v \in \text { VERTEX } \\
0 \leq e[v, w] \leq 2 t_{0} & (v, w) \in \text { EDGE } \\
0 \leq \alpha[v, F] \leq 2 \pi & (v, F) \in \text { ANGLE } \\
0 \leq \operatorname{sol}[F] \leq 4 \pi & F \in \text { FACE }
\end{array}
$$

There are other linear relations that are suggested directly by the definitions or the geometry. Here, $v$ belongs to VERTEX.

$$
\begin{array}{ll}
\tau[F] & =\operatorname{sol}[F] \zeta p t-\sigma[F], \\
2 \pi & =\sum_{F: v \in F} \alpha[v, F], \\
\operatorname{sol}[F] & =\sum_{v \in F} \alpha[v, F]-(\operatorname{len}(F)-2) \pi
\end{array}
$$

There are long lists of additional inequalities that come from interval arithmetic verifications. For example, the interval verifications of $[2$, Propositions 4.1-4.3] supply us with many linear inequalities relating the variables

$$
\begin{array}{lll}
\sigma[F], & \tau[F], & \alpha[v, F], \\
\operatorname{sol}[F], & y[v], & e[v, w]
\end{array}
$$

whenever $F^{I}$ is a single standard region having three sides. Similarly, [5, Section 4] gives inequalities for $\sigma[F]$ and related variables, when the length of $F$ is four. A complete list of inequalities that are used for triangular and quadrilateral faces is found in [5, Section 9].

For exceptional faces, we have an admissible weight function $w(F)$. According to definitions $w(F)=\tau[F] / p t$, so that the inequalities for the weight function can be expressed in terms of the linear program variables.

When the exceptional face is not an aggregate, then it also satisfies the inequalities of $[6$, Theorem 4.4].

\subsection{Face Refinements}

The linear program formulated in the previous section does not carry enough information in general to obtain the desired bounds. The paper [6] shows that exceptional standard regions have a great deal of internal structure. Until now in this paper, we have mostly ignored this internal structure. We will now make a detailed examination of this internal structure.

A refinement $\tilde{F}$ of a face $F$ of a plane graph $G$ is a set $\tilde{F}$ of faces such that 
1. The intersection of the vertex set of $G$ with that of $\tilde{F}$ is the set $F$.

2. $\tilde{F} \cup\left\{F^{o p}\right\}$ is a plane graph.

We use refinements of faces to describe the internal structure of faces.

We introduce indexing sets FACE- $\tilde{F}$, VERTEX- $\tilde{F}$, ANGLE- $\tilde{F}$, EDGE- $\tilde{F}$, the sets of faces, vertices, angles, and edges in $\tilde{F}$, respectively, analogous to those introduced for $G$.

We create variables $\pi[\tilde{F}]$, and indexed variables

$$
\operatorname{sol}\{\mathrm{FACE}-\tilde{F}\}, \quad \operatorname{sc}\{\mathrm{FACE}-\tilde{F}\} \quad \tau \mathrm{Sc}\{\mathrm{FACE}-\tilde{F}\},
$$$$
\alpha\{\operatorname{ANGLE}-\tilde{F}\}, \quad y\{\text { VERTEX }-\tilde{F}\}, \quad e\{\operatorname{EDGE}-\tilde{F}\} .
$$

(Variables with names " $y[v]$ " and " $e[v, w]$ " were already created for some $v, w \in$ VERTEX- $\tilde{F} \cap$ VERTEX. In these cases, we use the variables already created.)

Each vertex $v$ in the refinement will be interpreted either as a vertex $v^{I} \in U(D)$, or as the endpoint of an upright diagonal lying over the standard region $F^{I}$. We will interpret the faces of the refinement in terms of the geometry of the decomposition star $D$ variously as flat quarters, upright quarters, anchored simplices, and the other constructs of [6]. This interpretation depends on the context, and will be described in greater detail below.

Once the interpretation of faces is fixed, the interpretations are as before for the variable names introduced already: $y, e, \alpha$, sol. The lower and upper bounds for $\alpha$ and sol are as before. The lower and upper bounds for $y[v]$ are 2 and $2 t_{0}$ if $v^{I} \in U(D)$, but if $\left(0, v^{I}\right)$ is an upright diagonal, then the bounds are $\left[2 t_{0}, 2 \sqrt{2}\right]$. The lower and upper bounds for $e$ will depend on the context.

\subsection{Variables related to score}

The variables sc are a stand-in for the score $\sigma$ on a face. We do not call them $\sigma$ because the sum of these variables will not in general equal the variable $\sigma[F]$, when $\tilde{F}$ is a refinement of $F$ :

$$
\left[\sum_{F^{\prime} \in \tilde{F}} \mathrm{sc}\left[F^{\prime}\right] \neq \sigma[F]\right]
$$

We will use have a weaker relation:

$$
\sigma[F] \leq \sum_{F^{\prime} \in \tilde{F}} \operatorname{sc}\left[F^{\prime}\right]+\pi[\tilde{F}] .
$$

The variable $\pi[\tilde{F}]$ is called the penalty associated with the refinement $\tilde{F}$. (Penalties are discussed at length in Appendix 16.) The interpretations of sc and $\pi[\tilde{F}]$ are rather involved, and will be discussed on a case-by-case basis below. The interpretation of $\tau \mathrm{sc}$ follows from the identity:

$$
\tau \operatorname{sc}\left[F^{\prime}\right]=\operatorname{sol}\left[F^{\prime}\right] \zeta p t-\operatorname{sc}\left[F^{\prime}\right], \quad \forall F^{\prime} \in \tilde{F} .
$$

The interpretation of variables that follows might appear to be hodge-podge at first. However, they are obtained in a systematic way. We analyze the proofs and approximations in $[6]$, and define sc $[F]^{I}$ as the best penalty-free scoring approximation that is consistent with the given face refinement. here are the details.

If the subregion is a flat quarter, the interpretation of $\mathrm{sc}[F]$ is the function $\hat{\sigma}$, defined in Appendix 16. If the subregion is an upright quarter $Q$, the interpretation of $\mathrm{sc}[F]$ is the function $\sigma(Q)$ from [2]. If the subregion is an anchored simplex that is not an upright quarter, $\mathrm{sc}[F]$ is interpreted as the analytic Voronoi function vor if the simplex has type $C$ or $C^{\prime}$, and as vor $_{0}$ otherwise. (The types $A, B, C$ and $C^{\prime}$ are defined in [6, Sections 2.5-2.10].) Whether or not the simplex has type $C$, the inequality sc $[F] \leq 0$ is satisfied. In fact, if vor 0 scoring is used, we note that there are no quoins, and $\phi\left(1, t_{0}\right)<0$.

If the subregion is triangular, if no vertex represents an upright diagonal, and if the subregion is not a quarter, then $\mathrm{sc}[F]$ is interpreted as vor or vor $_{0}$ depending on whether the simplex has type $A$. In either case, the inequality $\mathrm{sc}[F] \leq$ vor $_{0}$ is satisfied.

In most other cases, the interpretation of $\mathrm{sc}[F]$ is vor $_{0}$. However, if $R$ is a heptagon or octagon, and $F$ has $\geq 4$ sides, then $\mathrm{sc}[F]$ is interpreted as vor $_{0}$ except on simplices of type $A$, where it becomes the analytic Voronoi function.

If $R$ is a pentagon or hexagon, and $F$ is a quadrilateral that is not adjacent to a flat quarter, then the interpretation of $\mathrm{sc}[F]$ is the actual score of the subregion over the subregion. In this case, the score $\sigma_{R}$ has a well-defined meaning for the quadrilateral, because it is not possible for an upright quarter in the $Q$-system to straddle the quadrilateral region and an adjacent region. Consequently, any erasing that is done can be associated with the subregion without ambiguity. By the results of [4], we have $\mathrm{sc}[F] \leq 0$. We also have $\mathrm{sc}[F] \leq \operatorname{vor}_{0}$.

One other bound that we have not explicitly mentioned is the bound $\sigma_{R}(D)<s_{n}$. For heptagons and octagons that are not aggregates, this is a better bound than the one used in the definition of tameness (Property 6). In heptagons and octagons 
that are not aggregates, if we have a subregion with four or more sides, then $\operatorname{sc}[F]<Z(n, k)$ and $\tau \mathrm{sc}[F]>D(n, k)$. (See $[6,5.5 .1],[6,5.5 .2]$.

The variables are subject to a number of compatibility relations that are evident from the underlying definitions and geometry.

$$
\begin{array}{ll}
\operatorname{sol}\left[F^{\prime}\right]=\sum_{v \in F^{\prime}} \alpha\left[v, F^{\prime}\right]-\left(\operatorname{len}\left[F^{\prime}\right]-2\right) \pi, & \forall F^{\prime} \\
\sum_{F^{\prime}: v \in F^{\prime}, F^{\prime} \in \mathrm{FACE}-\tilde{F}} \alpha\left[v, F^{\prime}\right]=\alpha[v, F], & \forall v
\end{array}
$$

Assume that a face $F_{1} \in \tilde{F}$ has been interpreted as a subregion $R=F_{1}^{I}$ of a standard region. Assume that each vertex of $F_{1}$ is interpreted as a vertex in $U(D)$ or as the endpoint of an upright diagonal over $F^{I}$. One common interpretation of sc is $\operatorname{vor}_{0, F}(U(D))$, the truncated Voronoi function. When this is the interpretation, we introduce further variables:

$$
\begin{array}{ll}
\operatorname{quo}\left[v, s\left(v, F_{1}\right)\right] & \forall v \in F_{1}, \\
\operatorname{quo}\left[s\left(v, F_{1}\right), v\right] & \forall v \in F_{1}, \\
\operatorname{Adih}\left[v, F_{1}\right] & \forall v \in F_{1},
\end{array}
$$

We interpret the variables as follows. If $w=s(v)$, and the triangle $\left(0, v^{I}, w^{I}\right)$ has circumradius $\eta$ at most $t_{0}$, then

$$
\begin{aligned}
\operatorname{quo}[v, w]^{I} & =\operatorname{quo}\left(R\left(\left|v^{I}\right| / 2, \eta, t_{0}\right)\right), \\
\operatorname{quo}[w, v]^{I} & =\operatorname{quo}\left(R\left(\left|w^{I}\right| / 2, \eta, t_{0}\right)\right) .
\end{aligned}
$$

If the circumradius is greater than $t_{0}$, we take

$$
\operatorname{quo}[v, w]^{I}=\operatorname{quo}[w, v]^{I}=0 .
$$

The variable Adih has the following interpretation:

$$
\operatorname{Adih}\left[v, F_{1}\right]^{I}= \begin{cases}A\left(\left|v^{I}\right| / 2\right) \alpha\left(v^{I}, F_{1}^{I}\right) & \left|v^{I}\right| \leq 2 t_{0} \\ 0 & \text { otherwise. }\end{cases}
$$

Under these interpretations, the following identity is satisfied:

$$
\begin{aligned}
\operatorname{sc}\left[F_{1}\right]= & \operatorname{sol}\left[F_{1}\right] \phi_{0}+\sum_{v \in F_{1}} \operatorname{Adih}\left[v, F_{1}\right] \\
& -4 \delta_{\text {oct }} \sum \mathrm{quo}[v, w] .
\end{aligned}
$$

This relation and the constants that appear in it are based on [5, Appendix A.4]. The final sum runs over all pairs $(v, w)$, where $v=s\left(w, F_{1}\right)$ or $w=s\left(v, F_{1}\right)$.

For this to be useful, we need good inequalities governing the individual variables. Such inequalities for $\operatorname{Adih}[v, F]$ and quo $[v, w]$ are found in $[5$, A.4]. To make of these inequalities, it is necessary to have lower and upper bounds on $\alpha[v, F]$ and $y[v]$. We obtain such bounds as LP-derived inequalities in the sense of Remark 10.3.

\subsection{Triangle and Quad Branching}

The paper [5, Appendix A.7] divides triangular faces into two cases:

$$
\begin{array}{ll}
y\left[v_{1}\right]+y\left[v_{2}\right]+y\left[v_{3}\right] & \leq 6.25 . \\
y\left[v_{1}\right]+y\left[v_{2}\right]+y\left[v_{3}\right] & \geq 6.25 .
\end{array}
$$

whenever sufficiently good bounds are not obtained as a single linear program. That paper also divides quadrilateral faces into four cases: two flat quarters, two flat quarters with diagonal running in the other direction, four upright quarters forming an octahedron, no quarters. In general, if there are $r_{1}$ triangles and $r_{2}$ quadrilaterals, we obtain as many as $2^{r_{1}+2 r_{2}}$ cases by breaking the various triangles and quadrilaterals into subcases.

We break triangular faces and quadrilaterals into subcases as needed in the linear programs that follow without further comment.

\section{Elimination of Aggregates}

The proof of the following theorem occupies the entire section. It eliminates all the pathological cases that we have had to carry along until now. The terminology in the next lemma comes from [6] (anchors, $\mathbf{S}_{3}^{-}, \mathbf{S}_{3}^{+}$, and so forth). This terminology is reviewed in Appendix 16.

Theorem 12.1. Let $D$ be a contravening decomposition star, and let $G$ be its tame graph. Every face of $G$ corresponds to exactly one standard region of $D$. No standard region of $D$ has any enclosed vertices from $U(D)$. (That is, enclosed vertices have height at least $2 t_{0}$. The decomposition star $D$ does not contain any $\mathbf{S}_{3}^{-}$or $\mathbf{S}_{4}^{+}$configurations. If an upright diagonal with at least 5 anchors appears, the 5 anchors are the five corners of $U(D)$ lying over a pentagonal standard region.

\subsection{A pentagonal hull with $n=8$}

The next few sections treat the nonpolygonal standard regions illustrated in Remark 4.2. In this subsection, there is an aggregate of the octagonal region and a triangle has a pentagonal hull. Let $P$ denote this aggregate.

Lemma 12.2. Let $G$ be a contravening plane graph with this aggregate. Some vertex on the pentagonal face has type not equal to $(3,0,1)$. 
Proof. If every vertex on the pentagonal face has type $(3,0,1)$, then at the vertex of the pentagon meeting the aggregated triangle, the four triangles together with the octagon give

$$
t_{8}+\sum_{(4)} \tau_{\mathrm{LP}}(4,0,2 \pi-2(1.153))>(4 \pi \zeta-8) p t,
$$

so that the graph does not contravene.

For a general contravening plane graph with this aggregate, we have bounds

$$
\begin{aligned}
& \sigma_{F}(D) \leq p t+s_{8} \\
& \tau_{F}(D) \geq t_{8} .
\end{aligned}
$$

We add the inequalities $\tau \mathrm{sc}[F]>t_{8}$ and $\mathrm{sc}[F]<$ $p t+s_{8}$ to the exceptional face. There is no other exceptional face, because $t_{8}+t_{5}>(4 \pi \zeta-8) p t$. We run the linear programs for all tame graphs with the property asserted by Lemma 12.2. Every upper bound is less than $8 \mathrm{pt}$, so that there are no contravening decomposition stars with this configuration.

\section{2 $n=8$, hexagonal hull}

We treat the two cases from Remark 4.2 that have a hexagonal hull (Figure 8). One can be described as a hexagonal region with an enclosed vertex that has height at most $2 t_{0}$ and distance at least $2 t_{0}$ from each corner over the hexagon. The other is described as a hexagonal region with an enclosed vertex of height at most $2 t_{0}$, but this time with distance less than $2 t_{0}$ from one of the corners over the hexagon.

The argument for the case $n=8$ with hexagonal hull is similar to the argument of Section 12.1. Add the inequalities $\tau(R)>t_{8}$ and $\sigma(R)<s_{8}$ for each hexagonal region. Run the linear programs for all tame graphs, and check that these additional inequalities yield linear programming bounds under 8 pt.

\section{3 $n=7$, pentagonal hull}

We treat the two cases illustrated in Figure 8 that have a pentagonal hull. One can be described as a pentagon with an enclosed vertex that has height at most $2 t_{0}$ and distance at least $2 t_{0}$ from each corner of the pentagon. The other is described as a pentagon with an enclosed vertex of height at most $2 t_{0}$, but this time with distance less than $2 t_{0}$ from one of the corners of the pentagon.
In discussing various maps, we let $v_{i}$ be the corners of the regions, and we set $y_{i}=\left|v_{i}\right|$ and $y_{i j}=\left|v_{i}-v_{j}\right|$. The subscript $F$ is dropped, when there is no great danger of ambiguity.

Add the inequalities $\tau[F]>t_{7}, \sigma[F]<s_{7}$ for the pentagonal face. There is no other exceptional region, because $t_{5}+t_{7}>(4 \pi \zeta-8) p t$. With these changes, of all the tame plane graphs with a pentagonal face, all but one of the linear programs give a bound under $8 p t$.

The plane graph $G_{15}$ that remains is easy to describe. It is the plane graph with 11 vertices, obtained by removing from an icosahedron a vertex and all the edges that meet at that vertex.

We treat the case $G_{15}$. Familiarity with Appendix 16 will be assumed in the following passage. Let $v_{12}$ be the vertex enclosed over the pentagon. We let $v_{1}, \ldots, v_{5}$ be the five corners of $U(D)$ over the pentagon. Break the pentagon into five simplices along $\left(0, v_{12}\right): S_{i}=\left(0, v_{12}, v_{i}, v_{i+1}\right)$. We have LP-derived bounds (in the sense of Remark 10.3) $y\left[v_{i}\right] \leq 2.168$, and $\alpha\left[v_{i}, F\right] \leq 2.89$, for $i=1,2,3,4,5$. In particular, the pentagonal region is convex.

Another LP-derived inequality is

$$
\sigma[F]>-0.2345 .
$$

Since -0.4339 is less than this the lower bound, the configuration $\mathbf{S}_{3}^{-}$does not occur. Similarly, since -0.25 is less than the lower bound, $\mathbf{S}_{4}^{+}$does not occur ([6, 3.7 and 3.8].

Suppose that there is a loop in context $(n, k)=$ $(4,2)$. Again the score is less than the LP-lower bound, showing that this does not occur:

$$
\sigma_{R}(D)<s_{7}+z_{\text {loop }}(4,2)<-0.2345 .
$$

The constants come from Table [6, Table 5.11] and [6, Theorem 4.4].

We have the LP-derived inequality

$$
\tau[F]<0.644 .
$$

If we branch and bound on the triangular faces, this LP-derived inequality can be improved to

$$
\tau[F]<0.6079 .
$$

If there is a loop other than $(4,2)$ and $(4,1)$, the linear program becomes infeasible:

$$
\tau[F]<0.644<t_{7}+\delta_{\text {loop }}(n, k)<\tau[F] .
$$


We conclude that all loops have context $(n, k)=$ $(4,1)$.

Case 1. The vertex $v_{12}$ has distance at least $2 t_{0}$ from the five corners of $U(D)$ over the pentagon.

The interval calculations relevant in Case 1 appear in Appendix 17.2.

The penalty to switch the pentagon to a pure vor $_{0}$ score is at most $5 \xi_{\Gamma}$ (see $\left.\left[6, \mathbf{A}_{10}\right]\right)$. There cannot be two flat quarters because then

$\left|v_{12}\right|>\mathcal{E}\left(S\left(2,2,2,2 t_{0}, 2 \sqrt{2}, 2 \sqrt{2}\right), 2 t_{0}, 2 t_{0}, 2 t_{0}\right)>2 t_{0}$.

(Case 1-a) Suppose there is one flat quarter, $\left|v_{1}-v_{4}\right| \leq 2 \sqrt{2}$. There is a lower bound of 1.2 on the dihedral angles of the simplices $\left(0, v_{12}, v_{i}, v_{i+1}\right)$. This is obtained as follows. The proof relies on the convexity of the quadrilateral region. We leave it to the reader to verify that the following pivots can be made to preserve convexity. Disregard all vertices except $v_{1}, v_{2}, v_{3}, v_{4}, v_{12}$. We give the argument that $\operatorname{dih}\left(0, v_{12}, v_{1}, v_{4}\right)>1.2$. The others are similar. Disregard the length $\left|v_{1}-v_{4}\right|$. We show that

$$
\begin{aligned}
s d & :=\operatorname{dih}\left(0, v_{12}, v_{1}, v_{2}\right)+\operatorname{dih}\left(0, v_{12}, v_{2}, v_{3}\right) \\
& +\operatorname{dih}\left(0, v_{12}, v_{3}, v_{4}\right)<2 \pi-1.2 .
\end{aligned}
$$

Lift $v_{12}$ so $\left|v_{12}\right|=2 t_{0}$. Maximize $s d$ by taking $\mid v_{1}-$ $v_{2}|=| v_{2}-v_{3}|=| v_{3}-v_{4} \mid=2 t_{0}$. Fixing $v_{3}$ and $v_{4}$, pivot $v_{1}$ around $\left(0, v_{12}\right)$ toward $v_{4}$, dragging $v_{2}$ toward $v_{12}$ until $\left|v_{2}-v_{12}\right|=2 t_{0}$. Similarly, we obtain $\left|v_{3}-v_{12}\right|=2 t_{0}$. We now have $s d \leq 3(1.63)<$ $2 \pi-1.2$, by an interval calculation.

Return to the original figure and move $v_{12}$ without increasing $\left|v_{12}\right|$ until each simplex $\left(0, v_{12}, v_{i}, v_{i+1}\right)$ has an edge $\left(v_{12}, v_{j}\right)$ of length $2 t_{0}$. Interval calculations show that the four simplices around $v_{12}$ squander

$2 \pi(0.2529)-3(0.1376)-0.12>(4 \pi \zeta-8) p t+5 \xi_{\Gamma}$.

(Case 1-b) Assume there are no flat quarters. An LP-derived bound on the perimeter $\sum\left|v_{i}-v_{i+1}\right|$ is 1.407. We have $\operatorname{arc}(2,2, x)^{\prime \prime}=2 x /\left(16-x^{2}\right)^{3 / 2}>0$. The arclength of the perimeter is therefore at most

$2 \operatorname{arc}\left(2,2,2 t_{0}\right)+2 \operatorname{arc}(2,2,2)+\operatorname{arc}(2,2,2.387)<2 \pi$.

There is a well-defined interior of the spherical pentagon, a component of area $<2 \pi$. If we deform by decreasing the perimeter, the component of area $<2 \pi$ does not get swapped with the other component.

Disregard all vertices but $v_{1}, \ldots, v_{5}, v_{12}$. If a vertex $v_{i}$ satisfies $\left|v_{i}-v_{12}\right|>2 t_{0}$, deform $v_{i}$ as in [6,
Section 4.9] until $\left|v_{i-1}-v_{i}\right|=\left|v_{i}-v_{i+1}\right|=2$, or $\left|v_{i}-v_{12}\right|=2 t_{0}$. If at any time, four of the edges realize the bound $\left|v_{i}-v_{i+1}\right|=2$, we have reached an impossible situation, because it leads to the contradiction (see Appendix 17.2)

$$
2 \pi=\sum^{(5)} \operatorname{dih}<1.51+4(1.16) .
$$

(This inequality relies on the observation, which we leave to the reader, that in any such assembly, pivots can by applied to bring $\left|v_{12}-v_{i}\right|=2 t_{0}$ for at least one edge of each of the five simplices.)

The vertex $v_{12}$ may be moved without increasing $\left|v_{12}\right|$ so that eventually by these deformations (and reindexing if necessary) we have $\left|v_{12}-v_{i}\right|=2 t_{0}$, $i=1,3,4$. (If we have $i=1,2,3$, the two dihedral angles along $\left(0, v_{2}\right)$ satisfy $<2(1.51)<\pi$, so the deformations can continue.)

There are two cases. In both cases $\left|v_{i}-v_{12}\right|=2 t_{0}$, for $i=1,3,4$.

(i) $\quad\left|v_{12}-v_{2}\right|=\left|v_{12}-v_{5}\right|=2 t_{0}$,

(ii) $\quad\left|v_{12}-v_{2}\right|=2 t_{0}, \quad\left|v_{4}-v_{5}\right|=\left|v_{5}-v_{1}\right|=2$,

Case (i) follows from interval calculations

$$
\sum \tau_{0} \geq 2 \pi(0.2529)-5(0.1453)>0.644+7 \xi_{\Gamma} .
$$

In case (ii), we have again

$$
2 \pi(0.2529)-5(0.1453)
$$

In this interval calculation we have assumed that $\left|v_{12}-v_{5}\right|<3.488$. Otherwise, setting $S=$ $\left(v_{12}, v_{4}, v_{5}, v_{1}\right)$, we have

$$
\Delta(S)<\Delta\left(3.488^{2}, 4,4,8,\left(2 t_{0}\right)^{2},\left(2 t_{0}\right)^{2}\right)<0,
$$

and the simplex does not exist. $\left(\left|v_{4}-v_{1}\right| \geq 2 \sqrt{2}\right.$ because there are no flat quarters.) This completes Case 1.

Case 2. The vertex $v_{12}$ has distance at most $2 t_{0}$ from the vertex $v_{1}$ and distance at least $2 t_{0}$ from the others.

Let $\left(0, v_{13}\right)$ be the upright diagonal of a loop $(4,1)$. The vertices of the loop are not $v_{2}, v_{3}, v_{4}, v_{5}$ with $v_{12}$ enclosed over $\left(0, v_{2}, v_{5}, v_{13}\right)$ by [6, Lemma 3.6]. The vertices of the loop are not $v_{2}, v_{3}, v_{4}, v_{5}$ with $v_{12}$ enclosed over $\left(0, v_{1}, v_{2}, v_{5}\right)$ because this would lead to a contradiction

$$
y_{12} \geq \mathcal{E}\left(S\left(2,2,2,2 t_{0}, 2 t_{0}, 3.2\right), 2 t_{0}, 2 t_{0}, 2\right)>2 t_{0},
$$


or

$$
y_{12} \geq \mathcal{E}\left(S\left(2,2,2,2 t_{0}, 2 t_{0}, 3.2\right), 2,2 t_{0}, 2\right)>2 t_{0} .
$$

We get a contradiction for the same reasons unless $\left(v_{1}, v_{12}\right)$ is an edge of some upright quarter of every loop of type $(4,1)$.

We consider two cases. (2-a) There is a flat quarter along an edge other than $\left(v_{1}, v_{12}\right)$. That is, the central vertex is $v_{2}, v_{3}, v_{4}$, or $v_{5}$. (Recall that the central vertex of a flat quarter is the vertex other than the origin that is not an endpoint of the diagonal.) (2-b) Every flat quarter has central vertex $v_{1}$.

Case 2-a. We erase all upright quarters including those in loops, taking penalties as required. There cannot be two flat quarters by geometric considerations

$$
\begin{aligned}
& \mathcal{E}\left(S\left(2,2,2,2 \sqrt{2}, 2 \sqrt{2}, 2 t_{0}\right), 2 t_{0}, 2 t_{0}, 2\right)>2 t_{0} \\
& \mathcal{E}\left(S\left(2,2,2,2 \sqrt{2}, 2 \sqrt{2}, 2 t_{0}\right), 2,2 t_{0}, 2 t_{0}\right)>2 t_{0}
\end{aligned}
$$

The penalty is at most $7 \xi_{\Gamma}$. We show that the region (with upright quarters erased) squanders $>7 \xi_{\Gamma}+0.644$. We assume that the central vertex is $v_{2}$ (case 2-a-i) or $v_{3}$ (case 2-a-ii). In case 2 -a-i, we have three types of simplices around $v_{12}$, characterized by the bounds on their edge lengths. Let $\left(0, v_{12}, v_{1}, v_{5}\right)$ have type $\mathrm{A},\left(0, v_{12}, v_{5}, v_{4}\right)$ and $\left(0, v_{12}, v_{4}, v_{3}\right)$ have type $\mathrm{B}$, and let $\left(0, v_{12}, v_{3}, v_{1}\right)$ have type $\mathrm{C}$. In case 2 -a-ii there are also three types. Let $\left(0, v_{12}, v_{1}, v_{2}\right)$ and $\left(0, v_{12}, v_{1}, v_{5}\right)$ have type $\mathrm{A},\left(0, v_{12}, v_{5}, v_{4}\right)$ type $\mathrm{B}$, and $\left(0, v_{12}, v_{2}, v_{4}\right)$ type $\mathrm{D}$. (There is no relation here between these types and the types of simplices $A, B, C$ defined in Appendix 16.2 and 16.3.) Upper bounds on the dihedral angles along the edge $\left(0, v_{12}\right)$ are given in Appendix 17.2. These upper bounds come as a result of a pivot argument similar to that establishing the bound 1.2 in Case 1-a.

These upper bounds imply the following lower bounds. In case 2-a-i,

$$
\begin{aligned}
& \operatorname{dih}>1.33 \quad(A), \\
& \operatorname{dih}>1.21 \quad(B), \\
& \operatorname{dih}>1.63 \quad(C),
\end{aligned}
$$

and in case $2-\mathrm{a}-\mathrm{ii}$,

$$
\begin{aligned}
& \text { dih }>1.37(A), \\
& \operatorname{dih}>1.25(B) \text {, } \\
& \text { dih }>1.51(D) \text {, }
\end{aligned}
$$

In every case the dihedral angle is at least 1.21. In case 2-a-i, the interval calculations of Appendix
17.2 lead to a lower bound on what is squandered by the four simplices around $\left(0, v_{12}\right)$. Again, we move $v_{12}$ without decreasing the score until each simplex $\left(0, v_{12}, v_{i}, v_{i+1}\right)$ has an edge satisfying $\left|v_{12}-v_{j}\right| \leq$ $2 t_{0}$. Interval calculations give

$$
\begin{aligned}
\sum_{(4)} \tau_{0} & >2 \pi(0.2529)-0.2391-2(0.1376)-0.266 \\
& >0.808
\end{aligned}
$$

In case 2-a-ii, we have

$$
\begin{aligned}
\sum_{(4)} \tau_{0} & >2 \pi(0.2529)-2(0.2391)-0.1376-0.12 \\
& >0.853 .
\end{aligned}
$$

So we squander more than $7 \xi_{\Gamma}+0.644$, as claimed.

Case 2-b. We now assume that there are no flat quarters with central vertex $v_{2}, \ldots, v_{5}$. We claim that $v_{12}$ is not enclosed over $\left(0, v_{1}, v_{2}, v_{3}\right)$ or $\left(0, v_{1}, v_{5}, v_{4}\right)$. In fact, if $v_{12}$ is enclosed over $\left(0, v_{1}, v_{2}, v_{3}\right)$, then we reach the contradiction

$$
\begin{aligned}
\pi & <\operatorname{dih}\left(0, v_{12}, v_{1}, v_{2}\right)+\operatorname{dih}\left(0, v_{12}, v_{2}, v_{3}\right) \\
& <1.63+1.51
\end{aligned}
$$

We claim that $v_{12}$ is not enclosed over $\left(0, v_{5}, v_{1}, v_{2}\right)$. Let $S_{1}=\left(0, v_{12}, v_{1}, v_{2}\right)$, and $S_{2}=$ $\left(0, v_{12}, v_{1}, v_{5}\right)$. We have the linear programming bound

$$
y_{4}\left(S_{1}\right)+y_{4}\left(S_{2}\right)=\left|v_{1}-v_{2}\right|+\left|v_{1}-v_{5}\right|<4.804 \text {. }
$$

An interval calculation gives (Appendix 17.3)

$$
\begin{aligned}
\sum_{(2)} \operatorname{dih}\left(S_{i}\right) & \leq \sum_{(2)}\left(\operatorname{dih}\left(S_{i}\right)+0.5\left(0.4804 / 2-y_{4}\left(S_{i}\right)\right)\right) \\
& <\pi
\end{aligned}
$$

So $v_{12}$ is not enclosed over $\left(0, v_{1}, v_{2}, v_{5}\right)$.

Erase all upright quarters, taking penalties as required. Replace all flat quarters with vor $_{0}$-scoring taking penalties as required. (Any flat quarter has $v_{1}$ as its central vertex.) We move $v_{12}$ keeping $\left|v_{12}\right|$ fixed and not decreasing $\left|v_{12}-v_{1}\right|$. The only effect this has on the score comes through the quoins along $\left(0, v_{1}, v_{12}\right)$. Stretching $\left|v_{12}-v_{1}\right|$ shrinks the quoins and increases the score. (The sign of the derivative of the quoin with respect to the top edge is computed in the proof of [6, Lemma 4.9].)

If we stretch $\left|v_{12}-v_{1}\right|$ to length $2 t_{0}$, we are done by case 1 and case 2 -a. (If deformations produce a flat quarter, use case 2-a, otherwise use case 1.) By the claims, we can eventually arrange (reindexing if necessary) so that

(i) $\quad\left|v_{12}-v_{3}\right|=\left|v_{12}-v_{4}\right|=2 t_{0}$, or

(ii) $\quad\left|v_{12}-v_{3}\right|=\left|v_{12}-v_{5}\right|=2 t_{0}$. 
We combine this with the deformations of $[6$, Section 4] so that in case (i) we may also assume that if $\left|v_{5}-v_{12}\right|>2 t_{0}$, then $\left|v_{4}-v_{5}\right|=\left|v_{5}-v_{1}\right|=2$ and that if $\left|v_{2}-v_{12}\right|>2 t_{0}$, then $\left|v_{1}-v_{2}\right|=\left|v_{2}-v_{3}\right|=2$. In case (ii) we may also assume that if $\left|v_{4}-v_{12}\right|>$ $2 t_{0}$, then $\left|v_{3}-v_{4}\right|=\left|v_{4}-v_{5}\right|=2$ and that if $\left|v_{2}-v_{12}\right|>2 t_{0}$, then $\left|v_{1}-v_{2}\right|=\left|v_{2}-v_{3}\right|=2$.

Break the pentagon into subregions by cutting along the edges $\left(v_{12}, v_{i}\right)$ that satisfy $\left|v_{12}-v_{i}\right| \leq 2 t_{0}$. So for example in case (i), we cut along $\left(v_{12}, v_{3}\right)$, $\left(v_{12}, v_{4}\right),\left(v_{12}, v_{1}\right)$, and possibly along $\left(v_{12}, v_{2}\right)$ and $\left(v_{12}, v_{5}\right)$. This breaks the pentagon into triangular and quadrilateral regions.

In case (ii), if $\left|v_{4}-v_{12}\right|>2 t_{0}$, then the argument used in Case 1 to show that $\left|v_{4}-v_{12}\right|<3.488$ applies here as well. In case (i) or (ii), if $\left|v_{12}-v_{2}\right|>2 t_{0}$, then for similar reasons, we may assume

$$
\Delta\left(\left|v_{12}-v_{2}\right|^{2}, 4,4,8,\left(2 t_{0}\right)^{2},\left|v_{12}-v_{1}\right|^{2}\right) \geq 0 .
$$

This justifies the hypotheses for the interval calculations in Appendix 17.2. We conclude that

$\sum \tau_{0} \geq 2 \pi(0.2529)-3(0.1453)-2(0.2391)>0.6749$

Recall that the LP-upper bound on what is squandered is 0.6079 . If the penalty is less than $0.067=$ $0.6749-0.6079$, we are done.

We have ruled out the existence of all loops except $(4,1)$. Note that a flat quarter with central vertex $v_{1}$ gives penalty at most 0.02 by [6, Lemma 3.11.3]. If there is at most one such a flat quarter and at most one loop, we are done:

$$
3 \xi_{\Gamma}+0.02<0.067 .
$$

Assume there are two loops of context $(n, k)=$ $(4,1)$. They both lie along the edge $\left(v_{1}, v_{12}\right)$, which precludes any unmasked flat quarters. If one of the upright diagonals has height $\geq 2.696$, then the penalty is at most $3 \xi_{\Gamma}+3 \xi_{V}<0.067$. Assume both heights are at most 2.696. The total internal angle of the exceptional face at $v_{1}$ is at least four times the dihedral angle of one of the flat quarters along $\left(0, v_{1}\right)$, or $4(0.74)$ by an interval calculation. This is contrary to the linear programming upper bound $(0.289)$ on the dihedral angle along $\left(0, v_{1}\right)$. This completes Case 2. This shows that heptagons with pentagonal hulls do not occur.

\section{$12.4 \mathrm{~S}_{3}^{-}, \mathrm{S}_{4}^{+}$}

We use the bounds on the score and on what is squandered from [6, Sections 3.7 and 3.8]: $\mathbf{S}_{4}^{+}(\sigma<$
$-0.25, \tau>-0.4), \mathbf{S}_{3}^{-}(\sigma<-0.4339, \tau>0.5606)$. If we have the configuration $\mathbf{S}_{3}^{-}$, there is only one exceptional region $\left(0.5606+t_{5}>(4 \pi \zeta-8) p t\right)$. The configuration with six standard regions around a vertex from Section 4.1 does not occur because a calculation with $\tau_{\mathrm{LP}}$ (see Section 6.1) show that the five quasi-regular tetrahedra in the configuration squander $>6 p t$, giving $6 p t+0.5606>(4 \pi \zeta-8) p t$.

We add the inequalities $\tau>0.5606$ and $\sigma<$ -0.4339 to the exceptional region. All linear programming bounds drop under $8 p t$ when these changes are made.

The configuration $\mathbf{S}_{4}^{+}$requires more work. We begin with a lemma.

Lemma 12.3. Let $\alpha$ be the dihedral angle along the large gap in an $\mathbf{S}_{4}^{+}$configuration. Let $v_{1}$ and $v_{2}$ be the anchors of $U(D)$ lying along the large gap. If $\left|v_{1}\right|+\left|v_{2}\right|<4.6$, then $\alpha>1.78$ and the score of the four quarters is at most -0.31547 .

Proof. The bound $\alpha>1.78$ is an interval calculation (Appendix 17.4). The upper bound on the score is a linear programming calculation involving the inequality $\alpha>1.78$ and the known inequalities on the score of an upright quarter.

Add the inequalities $\sigma<-0.25, \tau>0.4$ at the exceptional regions. The configuration $\mathbf{S}_{4}^{+}$does not appear in a pentagon. Run the linear programs for all tame plane graphs with an exceptional region that is not a pentagon. If this linear program fails to produce a bound of $8 \mathrm{pt}$, we use the lemma to branch into two cases: either $y\left[v_{1}\right]+y\left[v_{2}\right] \geq 4$.6, or $\sigma[R]<-0.31547$. In every case the bound drops below $8 p t$.

\subsection{Type $(n, k)=(5,1)$}

We return briefly to the case of six standard regions around a vertex discussed in Section 4.1. In the plane graph they are aggregated into an octahedron. We take each of the remaining cases with an octahedron, and replace the octahedron with a pentagon and six triangles around a new vertex. There are eight ways of doing this. All eight ways in each of the cases gives an LP bound under $8 \mathrm{pt}$. This completes this case. 


\subsection{Four anchors}

In the context $(n, k)=(4,3)$, the standard region $R$ must have at least seven sides $n(R) \geq 7$. Then

$$
\begin{aligned}
\tau(D) & \geq t_{7}+\delta_{\text {loop }}(4,3) \\
& >(4 \pi \zeta-8) p t
\end{aligned}
$$

Thus, we may assume that this context does not occur.

\subsection{Five Anchors}

Now turn to the decomposition stars with an upright diagonal with five anchors. Five quarters around a common upright diagonal in a pentagonal region can certainly occur. We claim that any other upright diagonal with five anchors leads to a decomposition star that does not contravene. In fact, the only other possible context is $(n, k)=(5,1)$ (see $[6$, 5.11]).

If this appears in an octagon, we have

$$
\tau(D)>\delta_{\text {loop }}(5,1)+t_{8}>(4 \pi \zeta-8) p t .
$$

If this appears in a heptagon, we have

$$
\tau(D)>\delta_{\text {loop }}(5,1)+t_{7}+0.55 p t>(4 \pi \zeta-8) p t
$$

because there must be a vertex that is not a corner of the heptagon. It cannot appear on a pentagon. If it appears on a hexagonal region $R$, we have

$$
\tau(D)>\delta_{\text {loop }}(5,1)+t_{6}
$$

and $\sigma(D)<z_{\text {loop }}(5,1)+s_{6}$. Add these constraints to the linear program of the tame graphs with a hexagonal face. The LP-bound on $\sigma(D)$ with these changes is $<8 p t$.

\section{Branching and Bound}

In Section 11.4 we mentioned that when the linear programs do not give sufficiently good bounds, we branch into various subcases depending on conditions triangles and quadrilaterals in the graph.

This section describes additional branching for exceptional regions. We assume the results from the Section 12 that eliminate the most unpleasant types of configurations.

\subsection{Pent and Hex Branching}

In Section 11.2 we discussed face refinements in abstract terms. We now describe these refinements in detail.

The possibilities are listed in the diagram only up to symmetry by the dihedral group action on the polygon. We do not prove the completeness of the list, but its completeness can be seen by inspection, in view of the comments that follow here and in Section 13.4.

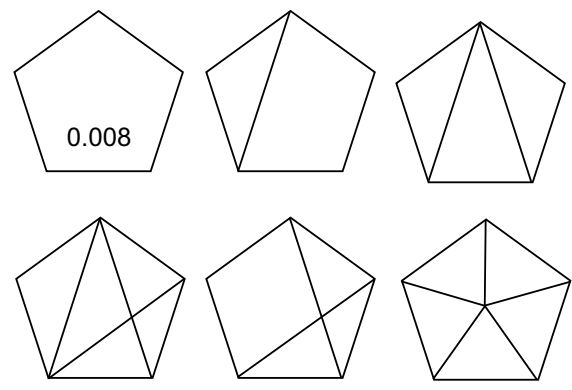

Figure 13: Pentagonal Face Refinements

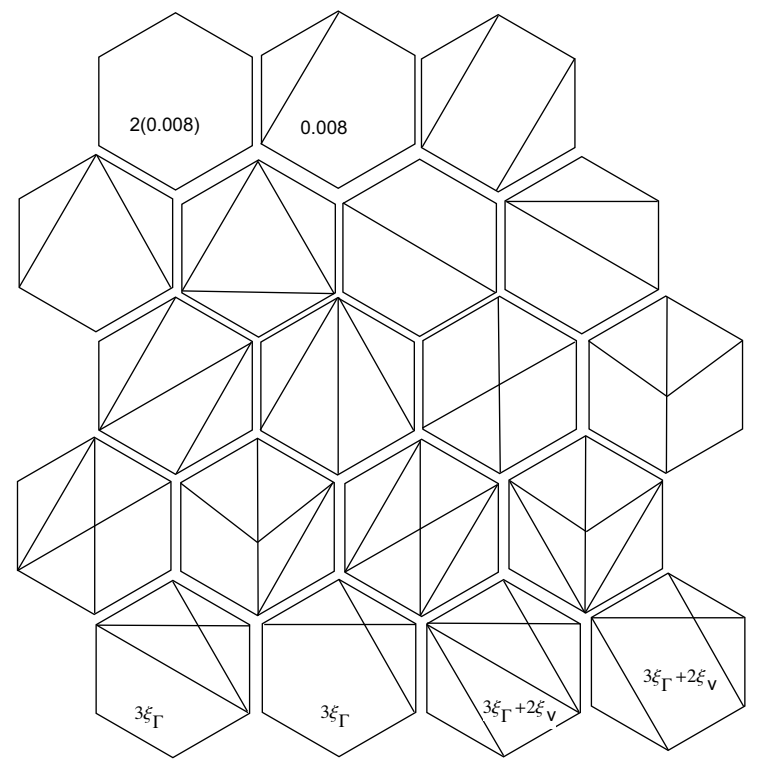

Figure 14: Hexagonal Face Refinements

The conventions for generating the possibilities are different for the pentagons and hexagons than for the heptagons and octagons. We describe the pentagons and hexagons first. We erase $\mathbf{S}_{3}^{+}$. If there is one loop we leave the loop in the figure. If there are two loops (so that both necessarily have context $(n, k)=(4,1))$, we erase one and keep the other. We draw all edges from an enclosed upright diagonal to its anchors, and all edges of length $\left[2 t_{0}, 2 \sqrt{2}\right]$ 
that are not masked by upright quarters. Since the only remaining upright quarters belong to loops, the four simplices around a loop are anchored simplices and the edge opposite the diagonal has length at most 3.2 .

\subsection{Branching on pentagonal faces}

The first three inequalities of Appendix 17.12 and the first inequality of Appendix 17.29 have been designed with pentagons in mind. The additional inequalities in Appendix 17.30, 17.31, 17.32, and the last two inequalities of 17.12 have been designed for subregions in hexagonal regions.

\subsection{Hept and Oct Branching}

When the figure is a heptagon or octagon, we proceed differently. We erase all $\mathbf{S}_{3}^{+}$configurations and all loops (either context $(n, k)=(4,1)$ or $(4,2))$ and draw only the flat quarters. An undrawn diagonal of the polygon has length at least $2 t_{0}$. Thus, in these cases much less internal structure is represented.

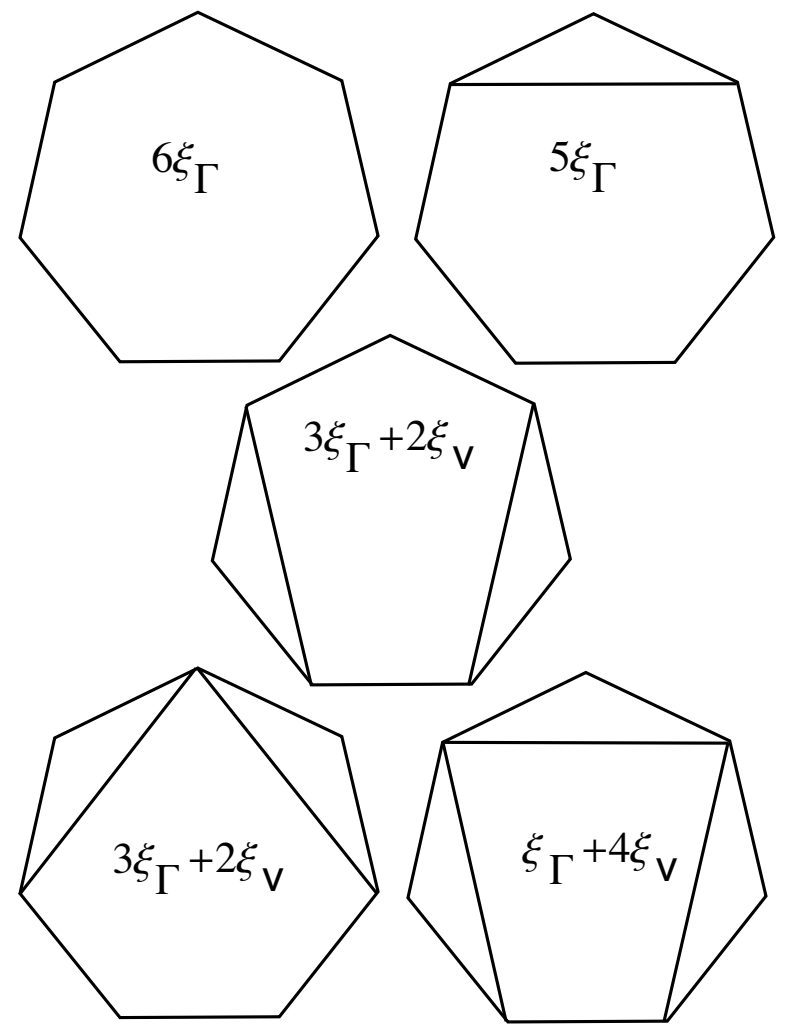

Figure 15: Hept Face Refinements

In the cases where $\mathbf{S}_{3}^{+}$or loops have been erased,

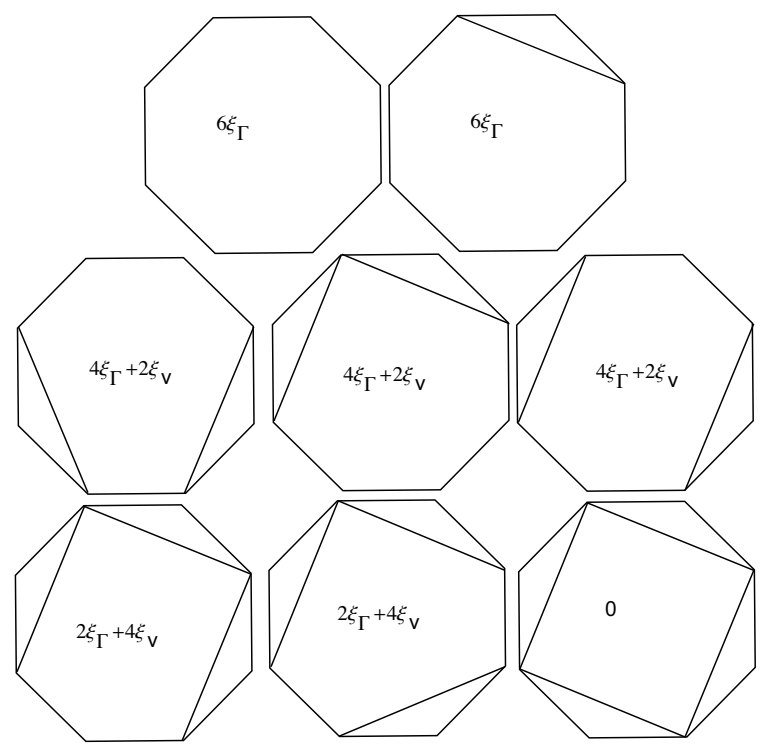

Figure 16: Oct Face Refinements

a number indicating a penalty accompanies the diagram. These penalties are derived in Section 13.4.

Here are some special arguments that are used for heptagons and octagons.

Suppose that the face refinement gives two subregions: a flat quarter and one with parameters $(n, k)=(n-1,1)$, with $n=6$ or 7 . We have the inequality:

$$
\sigma_{R}(D)<\hat{\sigma}+Z(n-1,1)+\xi_{\Gamma}+2 \xi_{V} .
$$

The penalty term $\xi_{\Gamma}+2 \xi_{V}$ comes from a possible anchored simplex that masks the flat quarter. Let $v$ be the central vertex of the flat quarter. Let $\left(v_{1}, v_{2}\right)$ be its diagonal. Masked flat quarters satisfy restrictive edge constraints. Thus, we have one of the following three possibilities:

1. $y[v] \geq 2.2$,

2. $y\left[v_{1}, v_{2}\right] \geq 2.7$,

3. $\sigma_{R}(D)<\hat{\sigma}+Z(n-1,1)$.

We proceed similarly, if the face refinement has three subregions: two flat quarters $R_{1}, R_{2}$ and one $\left(R_{3}\right)$ with parameters $(n, k)=(n-2,2)$. We have the cases:

1. The height of a central vertex is at least 2.2 .

2. The diagonal of a flat quarter is at least 2.7.

3.

$$
\begin{aligned}
& \sigma_{R}(D) \quad<\hat{\sigma}_{1}+\hat{\sigma}_{2}+Z(n-2,2) \\
& \tau_{R}(D) \quad>\hat{\tau}_{1}+\hat{\tau}_{2}+D(n-2,2)
\end{aligned}
$$


With heptagons, it is helpful on occasion to use an upper bound on the penalty of $3 \xi_{\Gamma}=0.04683$. This bound holds if neither flat quarter is masked by a loop. For this, it suffices to show that first two of the given three cases do not hold.

\subsubsection{Octagon Branching}

If there is a loop of context $(n, k)=(4,2)$, we have, by Lemma 16.4, the upper bound

$$
s_{n}+z_{\text {loop }}(4,2)
$$

on the score of exceptional standard regions $R$, with $n=n(R)$.

If there is no loop of context $(n, k)=(4,2)$, and if there are two flat quarters in the face refinement, we have the upper bound

$$
\hat{\sigma}_{1}+\hat{\sigma}_{2}+D(n-2,2)+2\left(\xi_{\Gamma}+2 \xi_{V}\right) .
$$

\subsection{Penalties}

The reader should review Appendix 16 before reading this section.

Erasing an upright quarter of compression type gives a penalty of at most $\xi_{\Gamma}$ and one of Voronoi type gives at most $\xi_{V}$. We take the worst possible penalty. It is at most $n \xi_{\Gamma}$ in an $n$-gon. If there is a masked flat quarter, the penalty is at most $2 \xi_{V}$ from the two upright quarters along the flat quarter. We note in this connection that both edges of a polygon along a flat quarter lie on upright quarters, or neither does.

If an upright diagonal appears enclosed over a flat quarter, the flat quarter is part of a loop with context $(n, k)=(4,1)$, for a penalty at most $2 \xi_{\Gamma}^{\prime}+$ $\xi_{V}$. This is smaller than the bound on the penalty obtained from a loop with context $(n, k)=(4,1)$, when the upright diagonal is not enclosed over the flat quarter:

$$
\xi_{\Gamma}+2 \xi_{V}
$$

So we calculate the worst-case penalties under the assumption that the upright diagonals are not enclosed over flat quarters.

A loop of context $(n, k)=(4,1)$ gives $\xi_{\Gamma}+2 \xi_{V}$ or $3 \xi_{\Gamma}$. A loop of context $(n, k)=(4,2)$ gives $2 \xi_{\Gamma}$ or $2 \xi_{V}$.

If we erase $\mathbf{S}_{3}^{+}$, there is a penalty of 0.008 (or 0 if it masks a flat quarter.) This is dominated by the penalty $3 \xi_{\Gamma}$ of context $(n, k)=(4,1)$.
Suppose we have an octagonal standard region. We claim that a loop does not occur in context $(n, k)=(4,2)$. If there are at most three vertices that are not corners of the octagon, then there are at most 12 quasi-regular tetrahedra, and the score is at most

$$
s_{8}+12 p t<8 p t .
$$

Assume there are more than three vertices that are not corners over the octagon. We squander

$$
t_{8}+\delta_{\text {loop }}(4,2)+4 \tau_{\mathrm{LP}}(5,0)>(4 \pi \zeta-8) p t .
$$

As a consequence, context $(n, k)=(4,2)$ does not occur.

So there are at most 2 upright diagonals and at most 6 quarters, and the penalty is at most $6 \xi_{\Gamma}$. Let $f$ be the number of flat quarters This leads to

$$
\pi_{F}= \begin{cases}6 \xi_{\Gamma}, & f=0,1, \\ 4 \xi_{\Gamma}+2 \xi_{V}, & f=2, \\ 2 \xi_{\Gamma}+4 \xi_{V}, & f=3, \\ 0, & f=4 .\end{cases}
$$

The 0 is justified by a parity argument. Each upright quarter occurs in a pair at each masked flat quarter. But there is an odd number of quarters along the upright diagonal, so no penalty at all can occur.

Suppose we have a heptagonal standard region. Three loops are a geometric impossibility. Assume there are at most two upright diagonals. If there is no context $(n, k)=(4,2)$, then we have the following bounds on the penalty

$$
\pi_{F}= \begin{cases}6 \xi_{\Gamma}, & f=0, \\ 4 \xi_{\Gamma}+2 \xi_{V}, & f=1, \\ 3 \xi_{\Gamma}, & f=2, \\ \xi_{\Gamma}+2 \xi_{V}, & f=3 .\end{cases}
$$

If an upright diagonal has context $(n, k)=(4,2)$, then

$$
\pi_{F}= \begin{cases}5 \xi_{\Gamma}, & f=0,1, \\ 3 \xi_{\Gamma}+2 \xi_{V}, & f=2, \\ \xi_{\Gamma}+4 \xi_{V}, & f=3 .\end{cases}
$$

This gives the bounds used in the diagrams of cases.

\subsection{Branching on Upright Diago- nals}

We divide the upright simplices into two domains depending on the height of the upright diagonal, using $y_{1}=2.696$ as the break point. We break the upright quarters into cases: 
1. The upright diagonal has height at most 2.696.

2. The upright diagonal $(0, v)$ has height at least 2.696, and some anchor $w$ along the flat quarter satisfies $|w| \geq 2.45$ and $|v-w| \geq 2.45$. (There is a separate case here for each anchor $w$.)

3. The upright diagonal $(0, v)$ has height at least 2.696, and every anchor $w$ along the flat quarter satisfies $|w| \leq 2.45$ and $|v-w| \leq 2.45$.

Many inequalities have been specially designed to hold on these smaller domains. They are included into the linear programming problems as appropriate.

When all the upright quarters can be erased, then the case for upright quarters follows from some other case without the upright quarters. An upright quarter can be erased in the following situations. If the upright quarter is compression type and $\geq 2.696$, then

$$
\nu_{\Gamma}<\operatorname{vor}_{0}
$$

$\left(\left[6, \mathbf{A}_{10}\right]\right)$. (If there are masked flat quarters, they become scored by $\hat{\sigma}$.) If upright quarters is of Voronoi type and the anchors $w$ satisfy $|w| \leq 2.45$ and $|v-w| \leq 2.45$, then the quarter can be erased:

$$
\nu<\operatorname{vor}_{0}
$$

In general, we only have the weaker inequality ([6, $\left.\left.\mathbf{A}_{11}\right]\right)$

$$
\nu<\operatorname{vor}_{0}+\xi_{V}, \quad \xi_{V}=0.003521 .
$$

\subsection{Branching on Upright Quarters}

In a pentagon or hexagon, consider an upright diagonal with three upright quarters, that is, context $(n, k)=(4,1)$. If the upright diagonal is at most 2.696, and if an upright quarter shares both faces along the upright diagonal with other upright quarters, then we may assume that the upright quarter has compression type. For otherwise, there is a face of circumradius at least $\sqrt{2}$, and hence two upright quarters of Voronoi type. The inequality

$$
\text { octavor }<\text { octavor }_{0}-0.008 \text {, }
$$

if $y_{1} \in\left[2 t_{0}, 2.696\right]$, and $\eta_{126} \geq \sqrt{2}$ shows that the upright quarters can be erased without penalty because $\xi_{\Gamma}<2(0.008)$. If erased, the case is treated as part of a different case.

This allows the inequalities for $\nu_{\Gamma}$ in Appendix 17.6 and 17.7 to be used. Furthermore, it can often be concluded that all three upright quarters have compression type. For this, we use the Inequalities 17.27 and 17.23, which can often be used to show that if the anchored simplex has a face of circumradius at least $\sqrt{2}$, then the linear programming bound on the score is less than $8 p t$.

\subsection{Branching on Flat Quarters}

There are a few other interval-based inequalities that are used in particular cases. Before an inequality that uses compression scoring is used on a flat quarter, it is necessary to verify that the quarter satisfies $y_{1} \leq 2.2, y_{4} \leq 2.7, \eta_{234}, \eta_{456} \leq \sqrt{2}$, to insure that the flat quarter has compression type. The circumradius is not a linear-programming variable, so its upper bound must be deduced from edge-length information.

Information about the internal structure of an exceptional face gives improvements to the constants $1.4 p t$ and $1.5 p t$ of Property 4 in the definition of admissible weight assignments. (The bounds remain fixed at $1.4 p t$ and $1.5 p t$, but these arguments allow us to specify more precisely what simplices contribute to these bounds.) These constants contribute to the bound on the $\tau(D)$ through the admissible weight assignment. Assume that at the vertex $v$ there are four tetrahedra and an exceptional face, and that the exceptional face has a flat quarter with central vertex $v$. The calculations of Section 7.4 show that the four quarters and exceptional squander at least $1.5 \mathrm{pt}$. If there is no flat quarter (masked or unmasked) whose first edge lies along $(0, v)$, then the four quasi-regular tetrahedra at $v$ squander at least $1.5 \mathrm{pt}$. We can make similar improvements when $\operatorname{tri}(v)=3$.

If we have LP-derived inequalities on a flat quarter whose corners $v_{i}$ have height at most 2.14, and if the diagonal has length less than 2.77, then the circumradius of the face containing the origin and diagonal is at most $\eta(2.14,2.14,2.77)<\sqrt{2}$. This allows us to combine the cases defining $\hat{\sigma}$ into three cases.

1. The simplex has compression type.

2. The diagonal has length $\leq 2.7$ and $\eta_{456} \geq \sqrt{2}$.

3. The diagonal has length $\geq 2.7$.

In the last two cases, the scoring is by vor $_{0}$. We use the specially tailored Inequalities 17.15 and 17.16 . 


\subsection{Branching on Type A simplices}

Suppose that the vertices of a triangular subregion come by projection from a simplex with vertices at the origin and in $U(D)$. Suppose that the simplex has two edges of length in $\left[2 t_{0}, 2 \sqrt{2}\right]$, say $y_{5}, y_{6} \in$ $\left[2 t_{0}, 2 \sqrt{2}\right], y_{4} \in\left[2,2 t_{0}\right]$. These may be simplices of type A. Suppose that one edge is at most 2.77. Such simplices fall into the following domains.

1. The simplex has type $A$, so that $y_{5}, y_{6} \in$ $\left[2 t_{0}, 2.77\right]$, and the scoring is vor, the analytic Voronoi function.

2. An edge (say $y_{6}$ ) of the face shared with the flat quarter has length $y_{6} \geq 2.77$.

3. The edges have lengths $y_{5}, y_{6} \in\left[2 t_{0}, 2.77\right]$, and $\eta_{456} \geq \sqrt{2}$.

In the last two cases, the scoring is by the truncated Voronoi function vor $_{0}$. We use the specially tailored Inequalities 17.17.

If there is an enclosed vertex of height at most $\sqrt{2}$, then we can use Inequalities 17.18.

\subsection{Branching on Quadrilateral subregions}

The inequalities of 17.27 hold for a quadrilateral subregion, if certain conditions are satisfied. One of the conditions is $y_{4} \in[2 \sqrt{2}, 3.0]$, where $y_{4}$ is a diagonal of the subregion. Since this diagonal is not one of the linear programming variables, these bounds cannot be verified directly from the linear program. Instead we use the interval calculation 17.13 which relates the desired bound $y_{4} \leq 3$ to the linear programming variables $\alpha[v, F], y_{2}, y_{3}, y_{5}$, and $y_{6}$.

\subsection{Conclusion}

By combinations of branching along the lines set forth in the preceding sections, a sequence of linear programs is obtained that establishes that $\sigma(D)$ is less than $8 p t$. For details of particular cases, the interested reader can consult the log files in [8], which record which branches are followed for any given tame graph. (For most tame graphs, a single linear program suffices.)

This completes the proof of the Kepler conjecture.

\section{Appendix. Colored Spaces}

It is claimed at various places that the optimization problem is that of a continuous function on a compact topological space. That is, the function $\sigma$ on the space $X$ of decomposition stars is continuous. The purpose of this appendix is to describe the sense in which this is true.

We begin with an example that should make things clear. Suppose that we have a discontinuous piecewise linear function on the unit interval $[-1,1]$, as in Figure 17. It is continuous, except at $x=0$.

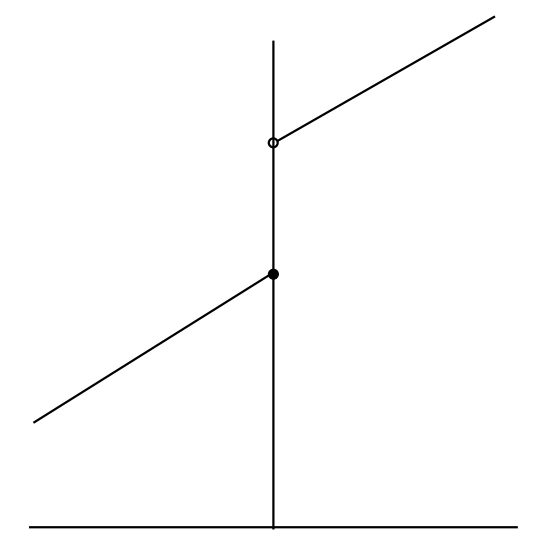

Figure 17: A piecewise linear function

We break the interval in two at $x=0$, forming two compact intervals $[-1,0]$ and $[0,1]$. We have a continuous functions $f_{-}:[-1,0] \rightarrow \mathbb{R}$ and $f_{+}$: $[0,1]$, such that

$$
f(x)= \begin{cases}f_{-}(x) & x \in[-1,0], \\ f_{+}(x) & \text { otherwise }\end{cases}
$$

We have replaced the discontinuous function by a pair of continuous functions on smaller intervals, at the expense of duplicating the point of discontinuity $x=0$. We view this pair of functions as a single function $F$ on the compact topological space with two components

$$
[-1,0] \times\{-\} \text { and }[0,1] \times\{+\} .
$$

where $F(x, a)=f_{a}(x)$, and $a \in\{-,+\}$.

This is the approach that we follow in general with the Kepler conjecture. The function $\sigma$ is defined by a series of case statements, and the function does not extend continuously across the boundary of the cases. However, in the degenerate cases that land precisely between two or more cases, we form 
multiple copies of decomposition star for each case, and place each case into a separate compact domain on which the function $\sigma$ is continuous.

This can be formalized as a colored space. A colored space is a topological space $X$ together with an equivalence relation on $X$ with the property that no point $x$ is equivalent to any other point in the same connected component as $x$. We refer to the connected components as colors, and call the points of $X$ colored points. We call the set of equivalence classes of $X$ the underlying uncolored space of $X$. Two colored points are equal as uncolored points if they are equivalent under the equivalence relation.

In our example, there are two colors "-" and "+." The equivalence class of $(x, a)$ is the set of pairs $(x, b)$ with the same first coordinate. Thus, if $x \neq 0$, the equivalence class contains one element $(x, \operatorname{sign}(x))$, and in the boundary case $x=0$ there are two equivalent elements $(0,-)$ and $(0,+)$.

In our treatment of the Kepler Conjecture, there are various cases: whether an edge has length less than or greater than $2 t_{0}$, less than or greater than $\sqrt{8}$, whether a face has circumradius less than or greater than $\sqrt{2}$, and so forth. By duplicating the degenerate cases (say an edge of exact length $2 t_{0}$ ), creating a separate connected component for each case, and expressing the optimization problem on a colored space, we obtain a continuous function $\sigma$ on a compact domain $X$.

The colorings have in general been suppressed from the notation of the paper. To obtain consistent results, a statement about $x \in\left[2,2 t_{0}\right]$ should be interpreted as having an implicit condition saying that $x$ has the coloring induced from the coloring on the component containing $\left[2,2 t_{0}\right]$. A later statement about $y \in\left[2 t_{0}, \sqrt{8}\right]$ deals with $y$ of a different color, and no relation between $x$ and $y$ of different colors is assumed at the endpoint $2 t_{0}$.

In general, to the greatest extent possible, we express each inequality as a strict inequality on a compact domain. There are, however, a few inequalities that are not strict, such as the bound of $1 p t$ on the score of a quasi-regular tetrahedron or the bound of 0 on the score of a quad cluster. (These particular sharp bounds appear in the proof of the local optimality of the face-centered cubic and hexagonal close packings.)

\section{Appendix. Constants and Functions}

This appendix gives a summary of various constants and functions that appear in the proof.

The density of a regular tetrahedron and Rogers's bound on the density of a packing is

$$
\delta_{\text {tet }}=\sqrt{8} \arctan (\sqrt{2} / 5) .
$$

The density of a regular octahedron is

$$
\delta_{\text {oct }}=\frac{\pi}{\sqrt{8}}-\frac{\delta_{t e t}}{2} \approx 0.72 .
$$

The unit of measure in calculations of $\sigma(D)$ is a point:

$$
p t=-\pi / 3+\sqrt{2} \delta_{t e t} \approx 0.05537 .
$$

A contravening decomposition star $D$ satisfies

$$
\sigma(D) \geq 8 p t
$$

or equivalently,

$$
\tau(D) \leq(4 \pi \zeta-8) p t
$$

where

$$
\zeta=1 /(2 \arctan (\sqrt{2} / 5))
$$

Objects are truncated using the value $t_{0}=1.255$. Centers of the packing at distance at most $2 t_{0}$ from the origin form a set $U(D)$.

Internal angles of triangular standard regions are at least 0.8638 , and those of other standard regions are at least 1.153. The internal angles of standard regions are generally denoted with variables $\alpha$. The dihedral angles of simplices $S$ are denoted $\operatorname{dih}(S)$. If the edge of a simplex has index $i$, then we write $\operatorname{dih}_{i}(S)$ for the dihedral angle along the edge with index $i$.

The circumradius of a triangle is denoted $\eta$. If there is a simplex with indexed edges, then $\eta_{i j k}$ denotes the circumradius of the face of the simplex whose edges have indices $i, j$, and $k$.

In general, the edges of simplices are indexed $1, \ldots, 6$ so that the first three edges meet at a vertex, and so that opposite edges have indices that are congruent $\bmod 3$. We write $y_{1}, \ldots, y_{6}$ for the edge lengths and $x_{i}=y_{i}^{2}$ as their squares. In general, the vertex where the first three edges meet is fixed as a distinguished vertex of the simplex. We write $\operatorname{sol}(S)$ for the solid angle of the simplex at that vertex. 
The function $\sigma$ on the topological space of decomposition stars is the function optimized in this paper. It is closely related to

$$
\tau(D)=\operatorname{sol}(D) \zeta p t-\sigma(D) .
$$

The function $\sigma$ is expressed as a sum of functions $\sigma_{R}$, indexed by standard regions. These sums are in turn expressed as finer sums.

The formulas for $\sigma_{R}$ and their upper bounds contain functions $\Gamma, \mu, \nu$, quo, Adih, vor, $\operatorname{vor}_{0}$. We do not repeat these definitions (they are found in [2]), because they appear in this paper in a purely formal way. (We make use of interval arithmetic inequalities that these functions satisfy, but we do not make use of anything deeper about them.) The function $\hat{\sigma}$ is defined in [6, Section 3.11]. The function $\operatorname{vor}_{x}$ is a function on anchored simplices that are not upright quarters. It is vor or $\operatorname{vor}_{0}$ depending on whether the upright simplex has type $C$. Also, we set

$$
\operatorname{vor}_{x}= \begin{cases}\text { vor } & \text { type } A, C, C^{\prime} \\ \operatorname{vor}_{0} & \text { otherwise }\end{cases}
$$

Corresponding to these functions related to $\sigma$ are functions related to $\tau$ :

$$
\begin{aligned}
\tau_{x} & =\operatorname{sol} \zeta p t-\operatorname{vor}_{x} \\
\tau_{R} & =\operatorname{sol} \zeta p t-\sigma_{R} \\
\hat{\tau} & =\operatorname{sol} \zeta p t-\hat{\sigma} \\
\tau & =\operatorname{sol} \zeta p t-\sigma \\
\tau_{0} & =\operatorname{sol} \zeta p t-\operatorname{vor}_{0} \\
\tau_{\Gamma} & =\operatorname{sol} \zeta p t-\Gamma \\
\tau_{\nu} & =\operatorname{sol} \zeta p t-\nu \\
\tau_{\mu} & =\operatorname{sol} \zeta p t-\mu \\
\tau_{V} & =\operatorname{sol} \zeta p t-\operatorname{vor} \\
\tau(, t) & =\operatorname{sol} \zeta p t-\operatorname{vor}(, t) .
\end{aligned}
$$

In the last case, the truncation is at $t=\sqrt{2}, 1.385$, $t_{0}$, and so forth. In each case, sol is the function that measures the solid angle of the object chosen from the domain of the function. Each function on the left-hand side is referred to as a squander, and each function that are subtracted on the right-hand side is a score. When the function $\tau_{*}$ is related to the scoring function in this way, we say that it is adapted.

The function vor $_{0}$ is used in many contexts, and we sometimes take liberties with the notation for this function. For example, we write $\operatorname{vor}_{0, R}(U(D))$ to indicate that the function depends only on the set $U(D)$ and the combinatorial information encoded by the region (or subregion) $R$.

The polynomial $\Delta$ of six variables has the property that $\Delta\left(x_{1}, \ldots, x_{6}\right)<0$, then the simplex with squared edge lengths $x_{i}$ does not exist. In the case of equality $\Delta=0$, the simplex degenerates to a planar quadrilateral.

\section{Appendix. A Review of [6]}

\subsection{Standard regions}

This paper relies heavily on results about the internal structure of standard regions that is developed in [6]. We make a brief review of these structures. We restrict our attention to exceptional standard regions. A quarter is a simplex, whose vertices are vertices $v \in \Lambda$ in the packing, such that five edges lengths are in $\left[2,2 t_{0}\right]$ and the sixth edge length lies in the interval $\left[2 t_{0}, \sqrt{8}\right]$. The sixth edge of the quarter is called its diagonal.

We fix our attention on the decomposition star $D$ centered at a given vertex in the packing, which we take to be located at the origin. The decomposition star determines the set $U(D)$ of all vertices of $\Lambda$ at distance at most $2 t_{0}$ from the origin.

From the decomposition star the set of quarters with a vertex at the origin can be also determined. There are two types. If the diagonal of the quarter has an endpoint at the origin, it is said to be upright. Otherwise, it is said to be flat. A flat quarter has its vertices at the origin and three points of the set $U(D)$. An upright quarter has one of its vertices $v$ outside $U(D)$. The edge $(0, v)$ is an upright diagonal.

The decomposition star determines a distinguished set of quarters with vertex at the origin. These distinguished quarters are said to belong to the $Q$-system. If one quarter along a diagonal lies in the $Q$-system, then every quarter along that diagonal lies in the $Q$-system. Quarters in the $Q$-system do not overlap (in the sense that they have disjoint interiors). The cones over the quarters at the origin in the $Q$-system do not overlap.

Each quarter lies entirely in the cone over a single standard region. We fix our attention on a single standard region and the quarters that are associated with it in this way. We write $U_{R}(D)$ for the subset of $U(D)$ consisting of points whose projection to the unit sphere lands in the (closed) region $R$.

There is a continuous function $\sigma$ defined on the topological space of all decomposition stars. It can be expressed as a sum of terms

$$
\sigma=\sum_{R} \sigma_{R}
$$


indexed by the set of standard regions. We will describe the structural properties of $\sigma_{R}$ for a given standard region $R$. There is a closely related function

$$
\tau_{R}=\operatorname{sol}(R) \zeta p t-\sigma_{R}
$$

The theory develops in parallel for these two functions.

\subsection{Flat quarters}

Flat quarters appear in several ways. We color (in the sense of Appendix 14) each quarter with identifying information that marks how that flat quarter arises. We will define functions $\hat{\sigma}$ and $\hat{\sigma}_{a d j}$ on the disjoint union of these different types of flat quarters.

If a flat quarter is in the $Q$-system, then we set

$$
\hat{\sigma}(S)=\hat{\sigma}_{a d j}(S)=\mu(S) .
$$

Where $\mu(S)$ is the function defined in [2, Formula 3.8].

Another type of flat quarter are those appearing in isolated pairs (see [2, Figure 1.10]). A special subset of such flat quarters are called simplices of type $B$. They have the following properties.

1. The simplex is a flat quarter.

2. The diagonal has length at least 2.77.

3. The edges running between the endpoints of the diagonal and the origin have length at most 2.23 .

For simplices of type $B$, set

$$
\hat{\sigma}(S)=\hat{\sigma}_{a d j}(S)=\operatorname{vor}(S, 1.385) .
$$

For simplices that are part of an isolated pair, but that are not of type $B$, we set

$$
\hat{\sigma}(S)=\hat{\sigma}_{a d j}(S)=\operatorname{vor}_{0}(S) .
$$

There are flat quarters that enclose an upright diagonal along an upright diagonal in the $Q$-system. We call these masked flat quarters of the first type. In this case, there are two upright quarters $Q_{1}$ and $Q_{2}$ over the flat quarter, as well as a third simplex $S$. We set

$$
\hat{\sigma}_{a d j}=\nu\left(Q_{1}\right)+\nu\left(Q_{2}\right)+\operatorname{vor}_{x}(S),
$$

where $\operatorname{vor}_{x}=$ vor if $S$ has type $C$, and $\operatorname{vor}_{x}=\operatorname{vor}_{0}$ otherwise. We note that $\eta_{456}(S) \geq \sqrt{2}$. Also, set

$$
\hat{\sigma}(S)=\min \left(\operatorname{vor}_{0}(S), 0\right) \text {. }
$$

We have

$$
\hat{\sigma}_{a d j} \leq \hat{\sigma}(S)
$$

\section{[6, Lemma 3.11.1]}

Let $\left(0, v, v_{1}, v_{2}\right)$ be a flat quarter that is not any of those discussed above. Let $v$ be the central vertex, and let $\left(v_{1}, v_{2}\right)$ be its diagonal. (Recall that the central vertex is defined as the vertex on a flat quarter that is not the origin and is not an endpoint of the diagonal.) These remaining flat quarters have the properties:

1. $\left|v_{1}-v_{2}\right| \geq 2.6$.

2. $\left|v_{1}-v_{2}\right| \geq 2.7$ or $|v| \geq 2.2$.

We call these quarters masked flat quarters of the second type. Set

$$
\begin{aligned}
& \epsilon= \begin{cases}0.0063 & |v| \geq 2.2, \\
0.0114 & |v| \leq 2.2 .\end{cases} \\
& \hat{\sigma}_{a d j}(S)=\operatorname{vor}_{0}(S)-\epsilon . \\
& \hat{\sigma}(S)=\operatorname{vor}_{0}(S) .
\end{aligned}
$$

For all types of flat quarters $S$, set

$$
\hat{\tau}(S)=\operatorname{sol}(S) \zeta p t-\hat{\sigma}(S)
$$

We have

$$
\begin{aligned}
& \hat{\sigma}_{a d j} \leq \hat{\sigma}, \\
& \hat{\sigma}_{a d j} \leq Z(3,1), \\
& \hat{\tau}_{a d j} \geq D(3,1),
\end{aligned}
$$

where $Z(3,1)=0.00005$ and $D(3,1)=0.06585$. (See $[6,5.5]$.)

\subsection{Type A}

A simplex $S$ of type $A$ is a simplex with a vertex at the origin and at three other points $v_{1}, v_{2}, v_{3}$ in $U_{R}(D)$ such that

1. The circumradius of the triangle with vertices $v_{1}, v_{2}, v_{3}$ is less than $\sqrt{2}$.

2. Two of the edges $\left(v_{i}, v_{j}\right)$ have edge length in the interval $\left[2 t_{0}, 2.77\right]$.

3. The third edge has length in the interval $\left[2,2 t_{0}\right]$. 


\subsection{Loops}

Let $(0, v)$ be an upright diagonal in the cone over a standard region $R$. We assume throughout this discussion that there is an upright quarter in the $Q$-system with diagonal $(0, v)$. A vertex $w$ (distinct from 0 and $v$ ) of distance at most $2 t_{0}$ from both 0 and $v$ is called an anchor (of $v$ ). By definition, we have $w \in U(D)$.

Lemma 16.1. The projection of $v$ to the unit sphere does not lie in the subregions associated with any flat quarter in the $Q$-system, any simplex of type $A$ or any simplex of type $B$. The projection $e$ of an edge $(v, w)$ does not meet the interior of a subregion associated with any flat quarter in the $Q$ system, a simplex of type $A$, or a simplex of type $B$.

The edges $(v, w)$ from $v$ to its anchors $w$ emanate like spokes from $v$. We order the anchors of $v$ according to this cyclic order into a cycle and write $s_{v}(w)$ for the successor of the anchor $w$ in this ordering. If the angle swept out along $(0, v)$ in passing from one spoke to the next is greater than $\pi$, we say that the angle around $(0, v)$ between $w$ and $s_{v}(w)$ is concave. Otherwise, we say it the angle is convex.

We say that a simplex $\left(0, v, w, s_{v}(w)\right)$ along $(0, v)$ is an anchored simplex, if the angle is convex and if $\left|w-s_{v}(w)\right| \leq 3.2$. We say that $\left(0, v, w, s_{v}(w)\right)$ is a large gap, if the angle is convex and if $\left|w-s_{v}(w)\right| \geq$ 3.2 .

Two other anchored simplices $S\left(y_{1}, \ldots, y_{6}\right)$ that arise are those of type $C$ or $C^{\prime}$. Write the anchored simplex $S$ with edge $y_{1}$ representing the length of the upright diagonal. We say the anchored simplex has type $C$ if $y_{4} \in\left[2 t_{0}, \sqrt{8}\right]$ and

1. $y_{4} \leq 2.77$, or

2. Both face circumradii of $S$ along the fourth edge are at most $\sqrt{2}$.

We say that the anchored simplex has type $C^{\prime}$ if

1. $y_{4} \in\left[2 t_{0}, \sqrt{8}\right]$,

2. $y_{2}, y_{6} \in\left[2.45,2 t_{0}\right]$,

3. The fourth edge is a diagonal of a flat quarter that shares a face with $S$.

If the anchored simplices around $(0, v)$ are not contained in a half-space bounded by a plane through $(0, v)$ and if there are no large gaps, then the anchored simplices around $v$ are said to form a loop around $v$. By construction, the anchored simplices around $v$ do not overlap. Anchored simplices (even those around different vertices) in a contravening decomposition star do not overlap. Anchored simplices do not overlap flat quarters in the $Q$-system, nor do they overlap simplices of types $A$ and $B$.

A special simplex is a simplex $\left(0, v_{1}, v_{2}, v_{3}\right)$ with vertices in $U_{R}(D)$ such that for some ordering of the indices, $\left|v_{1}-v_{2}\right| \leq 2 t_{0},\left|v_{2}-v_{3}\right| \leq 2 t_{0}$, and $\left|v_{1}-v_{3}\right| \in$ $[\sqrt{8}, 3.2]$. A special simplex $\left(0, v_{1}, v_{2}, v_{3}\right)$ is said to lie along an anchored simplex $\left(0, v, w, s_{v}(w)\right)$ if the simplices can be indexed so that $\left\{v_{1}, v_{3}\right\}=$ $\left\{w, s_{v}(w)\right\}$, with $\left|v_{1}-v_{3}\right| \geq \sqrt{8}$. Since anchored simplices do not overlap, each special simplex is related to at most one upright diagonal $(0, v)$ in this way. A special simplex attached to an upright diagonal in this way does not overlap any of the preceding objects (other special simplices so attached, other anchored simplices, simplices of types $A$ and $B$, and flat quarters in the $Q$-system).

\subsection{Bounds on $\sigma_{R}$ and $\tau_{R}$.}

Let $R$ be a standard region. More generally, we can partition $R$ into subregions by considering three types of bounding edges:

1. a bounding edge of a standard region,

2. the projection (to the unit sphere) of a face (along the diagonal) of an upright quarter in the $Q$-system,

3. the projection of a face formed by 0 and $v_{1}, v_{2} \in$ $U(D)$, where $\left|v_{1}-v_{2}\right| \leq \sqrt{8}$, and additionally if it bounds a flat quarter and that flat quarter is not in the $Q$-system then the face also bounds a simplex of type $A$.

Let $R$ be one of these subregions. We have

$$
\sigma(D)=\sum_{R} \sigma_{R}(D) .
$$

If $R$ is the projection of a simplex $Q$ in the $Q$ system, then $\sigma_{R}(D)=\sigma(Q)$, for some function $\sigma$ depending only on the simplex. Otherwise $\sigma_{R}(D)$ has the form

$$
\sigma_{R}(D)=\sigma_{R}\left(V_{D}\right),
$$

where $V_{D}$ is the $V$-cell defined in [2, Section 2]. The $V$-cell can be truncated at $\sqrt{2}$, which leads to the 
upper bound

$$
\sigma_{R}\left(V_{D}\right) \leq \operatorname{vor}_{R}\left(V_{D}, \sqrt{2}\right) \leq 0 .
$$

Each term indexed by quarters satisfies

$$
\sigma(Q) \leq 0 .
$$

In particular, if we have any subset $\mathcal{Q}$ of the set of quarters over a standard region $R$, then

$$
\sigma_{R}(D) \leq \sum_{\mathcal{Q}} \sigma(Q)
$$

There is a second partition of subregions that is used more frequently. Let $R$ be a subregion of the preceding paragraph. We break any anchored simplices that might occur from it (provided that the subregion does not come from a special simplex that lies along the anchored simplex). If this involves breaking the subregion into smaller pieces, we switch from the function $\sigma_{R}$ to the upper bound vor $_{0}$ (together with the volumes $\delta_{P}(v)$ of $[6,2.11]$ . We also break off any flat quarters that are not masked (we switch to the upper bound $\hat{\sigma}_{a d j}$ on the flat quarter and to $\operatorname{vor}_{0}$ on the rest of the subregion).

According to a construction that extends over several sections of [6] with a summary in $[6$, Section 3.10], it is often possible to eliminate the upright quarters around an upright diagonal by replacing the function $\sigma_{R}$ with an upper bound vor $_{0}$ over those pieces. This process is called erasing the upright quarters. At other times, we get a weaker statement, by replacing the functions $\sigma_{R}$ for regions $R$ at an upright diagonal $(0, v)$ with

$$
\sum_{R} \sigma_{R}(D) \leq \sum_{R} \operatorname{vor}_{0, R}(U(D))+\pi(v, D) .
$$

The constant $\pi(v, D)$ is called a penalty.

There are other upper bounds for $\sigma_{R}$ of interest. If we do not make approximations anywhere that produces penalties, we have a rather lengthy expression for $\sigma_{R}$ :

$$
\begin{aligned}
& \sigma_{R}(D)=\quad \sum_{Q} \hat{\sigma}(Q) \\
& +\sum_{A} \operatorname{vor}(S) \\
& +\sum_{B} \operatorname{vor}(S) \\
& +\sum_{\text {isolated, not } B} \operatorname{vor}_{0}(Q) \\
& +\sum_{\text {loop:v }} \sum_{S \mapsto v} \operatorname{vor}_{x}(S) \\
& +\sum_{\text {non-loop:v }}\left(\delta_{v}+\sum_{S \mapsto v} \operatorname{vor}_{x}(S)\right) \\
& +\sum_{\text {type } 1} \hat{\sigma}_{a d j} \\
& +\sum \operatorname{vor}_{0, R^{\prime}}\left(U_{R}(D)\right) \text {. }
\end{aligned}
$$

The sum indexed by $Q$ runs over flat quarters in the $Q$-system. The sum indexed by $A$ runs over simplices of type $A$ over $R$. The sum indexed by $B$ runs over simplices of type $B$ over $R$. The next sum runs over isolated flat quarters that do not have type $B$. The next sum runs over the upright diagonals $(0, v)$ of loops. (We restrict the sum to those loops that have four or five anchored simplices around it.) Its inner sum runs over the anchored simplices around $(0, v)$, as well as the special simplices that lie along these anchored simplices.

The next sum runs over upright diagonals $(0, v)$ of upright quarters in the $Q$-system that are not part of a loop. The next sum runs over masked flat quarters of type 1 for which there enclosed upright diagonal $(0, v)$ bounds exactly two quarters. The final sum is parameterized by subregions $R^{\prime}$ that have not otherwise been included, so that the subregions in the sum give a partition of $R$.

Each summand in this formula for $\sigma_{R}$ defines a geometric object whose cone cuts out a subregion $R^{\prime}$ on the unit sphere. We write $\sigma_{R^{\prime}}^{+}(D)$ for the summand. The summand is a single term except for those indexed by upright diagonals. The summands indexed by upright diagonals include the full inner sum over anchored simplices and so forth. We attach constants $(n, k)$ to each such subregion.

To each of these subregions, we associate a natural number $n(R)$. For polygons, it is the number of edges. The edge is to be counted with multiplicity two if it does not bound the standard region. We connect an internal dot that is not connected to any other vertices to another vertex by an imaginary edge of length greater than $2 t_{0}$. Such an imaginary edge is counted with multiplicity two. The numbers $n(R)$ are indicated in Figure 18. This constant is defined for the subregions indexing the sums of Identity 13.

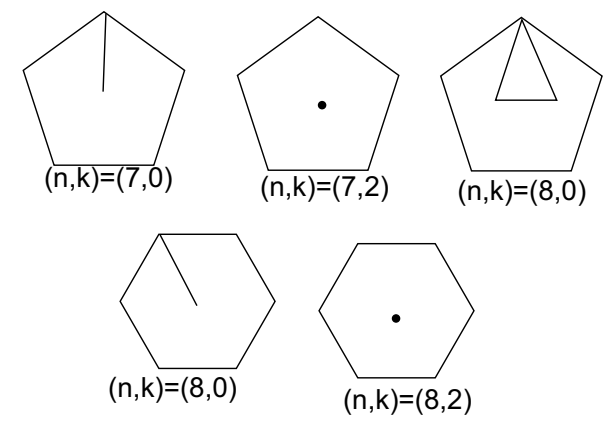

Figure 18: Parameters $(n, k)$ for non-polygonal regions 
A second parameter is $k(R)$ the number of sides of length at least $2 t_{0}$, counted with multiplicity 2 if it is internal. (Count the imaginary edge if it exists, with multiplicity two.)

Define constants

$$
\begin{array}{lll}
t_{4}=0.1317 & t_{5}=0.27113 & t_{6}=0.41056 \\
t_{7}=0.54999 & t_{8}=0.6045, & \\
s_{5}=-0.05704 & s_{6}=-0.11408 & s_{7}=-0.17112 \\
s_{8}=-0.22816 . & &
\end{array}
$$

Define constants

$$
D(n, k)=t_{n+k}-0.06585 k,
$$

for $0 \leq k \leq n$ and $t_{8} \geq n+k \geq 4$. It is readily verified that

$D\left(n_{1}, k_{1}\right)+D\left(n_{2}, k_{2}\right) \geq D\left(n_{1}+n_{2}-2, k_{1}+k_{2}-2\right)$.

Define constants $\epsilon=0.00005$ and

$$
Z(n, k)=s_{n+k}-k \epsilon,
$$

for $(n, k) \neq(3,1)$ and $Z(3,1)=\epsilon$. It is readily verified that

$Z\left(n_{1}, k_{1}\right)+Z\left(n_{2}, k_{2}\right) \leq Z\left(n_{1}+n_{2}-2, k_{1}+k_{2}-2\right)$.

Theorem 16.2. For subregions $R^{\prime}$ associated with summands as above, we have

$$
\begin{array}{ll}
\sigma_{R^{\prime}}^{+} & \leq Z\left(n\left(R^{\prime}\right), k\left(R^{\prime}\right)\right), \\
\operatorname{sol}\left(R^{\prime}\right) \zeta p t-\sigma_{R^{\prime}}^{+} & \geq D\left(n\left(R^{\prime}\right), k\left(R^{\prime}\right)\right) .
\end{array}
$$

\subsection{Bounds for Standard Regions}

Every standard region of a contravening decomposition star is a polygon with between 3 and 8 sides or one of the cases of Figure 18. In these figures, we draw a dot to indicate the projection of a vertex in $U(D)$ that does not lie on the boundary. These enclosed dots do not lie in the cone over a simplex of type $A$, nor in the cone over a quarter. We draw an edge between vertices if the corresponding vertices in $U(D)$ have distance at most $2 t_{0}$ from each other. (This figure is a copy of Figure 8.)

Corollary 16.3. Define constants as in Section 16.5. For every standard region $R$ such that $n=$ $n(R) \geq 5$ :

$$
\begin{aligned}
& \sigma_{R} \leq s_{n} \\
& \tau_{R} \geq t_{n}
\end{aligned}
$$

The corollary is an immediate consequence of the additivity properties of $\sigma_{R^{\prime}}^{+}$, the sub and superadditivity of $Z$ and $D$, and finally the geometric interpretation of sub and superadditivity as gluing subregions together to form a larger subregion. See [6, Section 4.5].
Lemma 16.4. Let $R$ be a subregion cut out by a loop group. Assume that the subregion is not that defined by a flat quarter. Let $n$ be the number of anchors in the loop. Let $k$ be the number of anchored simplices, with an edge (opposite the upright diagonal) of length at least $2 t_{0}$. Let $s$ be the number of special simplices. In a contravening plane graph $(n, k)$ is one of $(4,0),(4,1),(4,2),(4,3),(5,0)$, $(5,1)$. Furthermore,

$\operatorname{sol}(R) \zeta p t-\sigma_{R}^{+}(D) \geq D(n+s, k-s)+\delta_{\text {loop }}(n, k)$,

where $\delta_{\text {loop }}$ is defined by the following constants. Similarly,

$$
\sigma_{R}^{+}(D) \leq Z(n+s, k-s)+z_{\text {loop }}(n, k)
$$

$$
\begin{array}{cc}
(n, k) & \delta_{\text {loop }} \\
(4,0) & 0.0045 \\
(4,1) & 0.00272 \\
(4,2) & 0.12034 \\
(4,3) & 0.29426 \\
(5,0) & 0.09537 \\
(5,1) & 0.24939 \\
& \\
(n, k) & z_{\text {loop }} \\
(4,2) & -0.08582
\end{array}
$$

(see [6, Section 5.11].)

\subsection{Remaining subregions}

A masked flat quarter is a flat quarter that is not in the $Q$-system and that overlaps some anchored simplex around an upright diagonal $(0, v)$. The vertices of a masked flat quarter consist of three consecutive anchors of $v$

There are two cases to consider. Either the upright diagonal $(0, v)$ lies in the cone over the masked flat quarter or it does not. If it lies in the cone over the masked flat diagonal, then the following facts are known. (Recall that we are assuming throughout this appendix that the standard region is not a quadrilateral.) As above, we call these masked flat quarters of the first type.

1. The circumradius of the top face of the flat quarter is at least $\sqrt{2}$.

2. The anchored simplices form a loop.

3. The vertex $v$ has three or four anchors. 
4. If the vertex has three anchors, the loop consists of three anchored simplices that cut out the same region on the unit sphere as the masked flat quarter.

5. If the vertex has four anchors, the loop consists of three upright quarters and one other that is not an upright quarter.

If the upright diagonal does not lie in the cone over the masked flat quarter, then the following facts are known. Call these masked flat quarters of the second type.

1. The flat quarter has a diagonal of length at least 2.6 .

2. If the flat quarter has a diagonal of length less than 2.7, then the edge opposite the diagonal has length at least 2.2 .

\subsection{Penalties}

Set

$$
\pi_{\Gamma}(y)= \begin{cases}\xi_{\Gamma}=0.01561 & 2 t_{0} \leq y \leq 2.57 \\ \xi_{\Gamma}^{\prime}=0.00935 & 2.57 \leq y \leq \sqrt{8}\end{cases}
$$

and

$$
\pi_{V}(y)= \begin{cases}0 & 2 t_{0} \leq y \leq 2.57 \\ \xi_{V}=0.003521 & 2.57 \leq y \leq \sqrt{8}\end{cases}
$$

If $R$ is one of the subregions of Identity 13, then we have a two functions $\operatorname{vor}_{0, R}(U(D))$ and $\sigma_{R}^{+}(D)$. For example, $R$ may be the projection of the set of anchored simplices around an upright diagonal $(0, v)$, together with the set of special simplices that lie along the anchored simplices. In this situation, we write $\pi(v, D)$ for the difference:

$$
\sigma_{R}^{+}(D)=\operatorname{vor}_{0, R}(U(D))+\pi(v, D) .
$$

If $(0, v)$ is associated with a loop with $n$ anchored simplices, then we have

$$
\pi(v, D) \leq n \pi_{\Gamma}(|v|) .
$$

If there is a loop around $v$ with $n$ anchored simplices and with $k$ masked flat quarters of the second type, then

$$
\pi(v, D) \leq(n-2 k) \pi_{\Gamma}(|v|)+2 k \pi_{V}(|v|) .
$$

We recall that for masked flat quarters $Q$ of the second type, with projection to the subregion $R$, we have

$$
\hat{\sigma}(Q)=\operatorname{vor}_{0}(Q) .
$$

If there is a loop around $v$ with a masked flat quarter of the first type, then the context is $(n, k)=$ $(3,0)$ or $(4,1)$. In the case of context $(4,1)$, we have

$$
\pi(v, D) \leq \pi_{V}(|v|) .
$$

The constants $\pi(v, D)$ are called penalties. When we approximate $\sigma_{R}(D)$ by means of an upper bound on $\pi(v, D)$, the upright diagonal is said to be erased.

\subsection{Anchored Simplices}

Often, $\pi(v, D) \leq 0$. When this is the case, we generally eliminate (that is, erase) the upright diagonal and all the anchored simplices around it. (This has been done implicitly already.) When we do this, we use the function vor $_{0}$ on the region that becomes "exposed" in the process. It is possible to eliminate the upright diagonals in all cases, except

1. loops,

2. a configuration $\mathbf{S}_{3}^{+}$,

3. a configuration $\mathbf{S}_{3}^{-}$,

4. a configuration $\mathbf{S}_{4}^{+}$.

\subsubsection{The configuration $\mathrm{S}_{3}^{+}$}

The configuration $\mathbf{S}_{3}^{+}$around an upright diagonal $(0, v)$ consists of two upright quarters, one other anchored simplex, and one large gap. The configuration $\mathbf{S}_{3}^{+}$never masks a flat quarter. If the other anchored simplex is not an upright quarter, then the penalty satisfies

$$
\pi(v, D) \leq 0.00222 .
$$

If all three anchored simplices are upright quarters, then the penalty satisfies

$$
\pi(v, D) \leq 0.008 .
$$

\subsubsection{The configuration $\mathrm{S}_{3}^{-}$}

The configuration $\mathbf{S}_{3}^{-}$around an upright diagonal $(0, v)$ consists of three upright quarters that all lie in a common half-space bounded by a plane through 
$(0, v)$. If the configuration masks a flat quarter, then the penalty satisfies

$$
\pi(v, D) \leq \pi_{\Gamma}(|v|)+2 \pi_{V}(|v|) .
$$

If it does not mask a flat quarter, then

$$
\pi(v, D) \leq 3 \pi_{\Gamma}(|v|) .
$$

There is at most one $\mathbf{S}_{3}^{-}$configuration in a contravening decomposition star. Each upright quarter $Q$ is assigned a score $\sigma(Q)$. The three quarters satisfy

$$
\begin{aligned}
& \sum_{(3)} \sigma(Q) \quad<-0.4339 \\
& \sum_{(3)} \tau(Q) \quad>0.5606
\end{aligned}
$$

\subsubsection{The configuration $\mathrm{S}_{4}^{+}$}

The configuration $\mathbf{S}_{4}^{+}$around an upright diagonal $(0, v)$ consists of four upright quarters, and a large gap. The configuration masks at most $k \leq 2$ flat quarters. The penalty satisfies

$$
\pi(v, D) \leq 2 k \pi_{V}(|v|)+(4-2 k) \pi_{\Gamma}(|v|) .
$$

There is at most one $\mathbf{S}_{4}^{+}$configuration in a contravening decomposition star. It does not appear in a contravening decomposition star with $\mathbf{S}_{3}^{-}$. Each upright quarter $Q$ is assigned a score $\sigma(Q)$. The four quarters satisfy

$$
\begin{aligned}
& \sum_{(4)} \sigma(Q) \quad<-0.25 \\
& \sum_{(4)} \tau(Q)>0.4 .
\end{aligned}
$$

\subsection{Compatibility Notes}

We conclude this section with a few comments about differences in notation between this article and the others.

In [6], simplices of types $A, B, C$, and $C^{\prime}$ are called $S_{A}, S_{B}, S_{C}$, and $S_{D}$, respectively. The functions $\sigma_{R}(D)$ and $\tau_{R}(D)$ are denoted $\sigma(R)$ and $\tau(R)$, respectively, in other articles. The truncations $\operatorname{vor}_{0, R}(D)$ and $\tau_{0, R}(D)$ are denoted $\operatorname{vor}_{0}(R)$ and $\tau_{0}(R)$, respectively, in other articles. 


\section{Appendix. Calculations}

The proof of the Kepler Conjecture relies on a large number of inequalities that have been established by interval arithmetic with a computer. Generally, speaking there is no geometry of interest associated with these inequalities. The methods that are used in verifying these conjecture are described in [3] and [9]. (The paper describing the algorithms goes beyond what is used in these verifications, because it also proposes some new unimplemented techniques that should simplify the verification process.)

Interval calculations are arranged according to the section in which they appear. Each inequality is accompanied by one or more reference numbers. These identification numbers are needed to find further details about the calculation in [3]. Most of the verifications were completed by Samuel Ferguson. ${ }^{1}$ The verifications that he made are marked with a dagger $(\dagger)$. The computer-based proofs are carried out by interval arithmetic as in our earlier papers. A few inequalities are not standard in the sense that they have substantially higher dimension that the others. Linear programming methods are used to break these problems into six-dimensional pieces according to the suggestion of [5, Appendix 2].

Edge lengths whose bounds are not specified are assumed to be between 2 and $2 t_{0}$. The first edge of an upright quarter is its diagonal. The fourth edge of a flat quarter is its diagonal.

$\hat{\sigma}$ is the function of [6, Section 3.11] and $\hat{\tau}=$ $\operatorname{sol} \zeta p t-\hat{\sigma}$. The function definitions are those appearing in Appendix 15. The other functions are described in Appendix 15.

We introduce some notation for the heights and edge lengths of a polygon. The heights will generally be between 2 and $2 t_{0}$, the edge lengths between consecutive corners will generally be $2,2 t_{0}$, or $2 \sqrt{2}$. We represent the edge lengths by a vector

$$
\left(a_{1}, b_{1}, a_{2}, b_{2}, \ldots, a_{n}, b_{n}\right),
$$

if the corners of an $n$-gon, ordered cyclically have heights $a_{i}$ and if the edge length between corner $i$ and $i+1$ is $b_{i}$. Figure 19 illustrates the case $n=5$. We say two vectors are equivalent if they are related by a different cyclic ordering on the corners of the polygon, that is, by the action of the dihedral group.

\footnotetext{
${ }^{1}$ I express my sincere thanks to Samuel Ferguson for his fundamental contributions to this project.
}

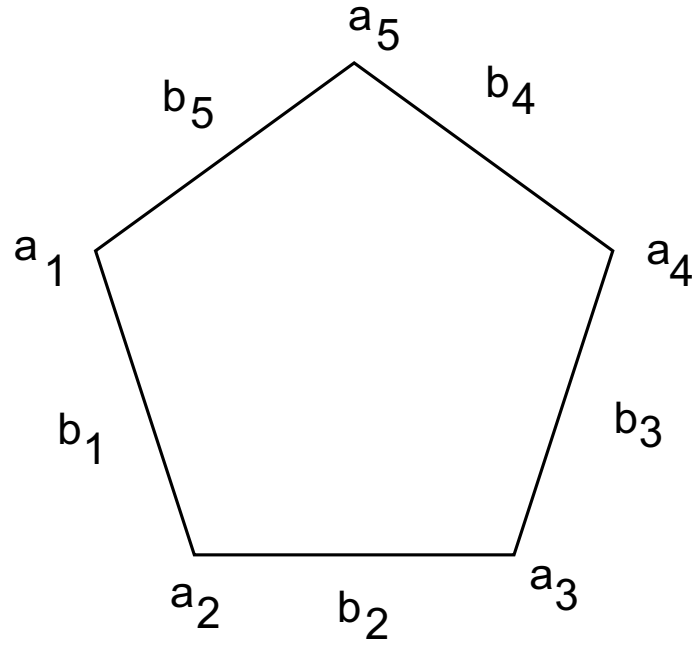

Figure 19: The labels on an $n$-gon. 


\subsection{Group 1}

$\tau-0.2529 \mathrm{dih}>-0.3442$, if $y_{1} \in\left[2.3,2 t_{0}\right]$, and $\operatorname{dih} \geq 1.51$.

(572068135)

$\tau_{0}-0.2529$ dih $>-0.1787$, if $y_{1} \in\left[2.3,2 t_{0}\right], y_{6} \in[2 \sqrt{2}, 3.02], 1.26 \leq \operatorname{dih} \leq 1.63$.

(723700608)

$\hat{\tau}-0.2529 \mathrm{dih}_{2}>-0.2137$, if $y_{2} \in\left[2.3,2 t_{0}\right], y_{4} \in\left[2 t_{0}, 2 \sqrt{2}\right]$,

(560470084)

$\tau_{0}-0.2529 \operatorname{dih}>-0.1371$, if $y_{1} \in\left[2.3,2 t_{0}\right], y_{5}, y_{6} \in\left[2 t_{0}, 3.02\right], 1.14 \leq \operatorname{dih} \leq 1.51$.

(535502975)

\subsection{Group $2 \dagger$}

A. $\operatorname{dih}<1.63$, if $y_{6} \geq 2 t_{0}, y_{2}, y_{3} \in[2,2.168]$.

(821707685)

B. $\operatorname{dih}<1.51$, if $y_{5}=2 t_{0}, y_{6} \geq 2 t_{0}, y_{2}, y_{3} \in[2,2.168]$.

(115383627)

C. $\operatorname{dih}<1.93$, if $y_{6} \geq 2 t_{0}, y_{4}=2 \sqrt{2}, y_{2}, y_{3} \in[2,2.168]$.

(576221766)

D. $\operatorname{dih}<1.77$, if $y_{5}=2 t_{0}, y_{6} \geq 2 t_{0}, y_{4}=2 \sqrt{2}, y_{2}, y_{3} \in[2,2.168]$.

(122081309)

$\tau_{0}-0.2529 \mathrm{dih}>-0.2391$, if $y_{6} \geq 2 t_{0}$, dih $\geq 1.2, y_{2}, y_{3} \in[2,2.168]$.

(644534985)

$\tau_{0}-0.2529 \mathrm{dih}>-0.1376$, if $y_{5}=2 t_{0}, y_{6} \geq 2 t_{0}$, dih $\geq 1.2$, and $y_{2}, y_{3} \in[2,2.168]$.

(467530297)

$\tau_{0}-0.2529 \mathrm{dih}>-0.266$, if $y_{6} \geq 2 t_{0}, y_{4} \in\left[2 t_{0}, 2 \sqrt{2}\right], \operatorname{dih} \geq 1.2, y_{2}, y_{3} \in[2,2.168]$.

(603910880)

$\tau_{0}-0.2529 \mathrm{dih}>-0.12$, if $y_{5}=2 t_{0}, y_{6} \geq 2 t_{0}, y_{4} \in\left[2 t_{0}, 2 \sqrt{2}\right], \operatorname{dih} \geq 1.2, y_{2}, y_{3} \in[2,2.168]$. (135427691)

$\operatorname{dih}<1.16$, if $y_{5}=2 t_{0}, y_{6} \geq 2 t_{0}, y_{4}=2, y_{2}, y_{3} \in[2,2.168]$.

(60314528)

$\tau_{0}-0.2529 \mathrm{dih}>-0.1453$, if $y_{2}, y_{3} \in[2,2.168], y_{5} \in\left[2 t_{0}, 3.488\right], y_{6}=2 t_{0}$.

(312132053)

\subsection{Group 3}

$\operatorname{dih}_{2}>0.74$, if $y_{1} \in\left[2 t_{0}, 2.696\right], y_{2}, y_{3} \in[2,2.168]$.

(751442360)

$\tau_{0}-0.2529 \mathrm{dih}>-0.2391$, if $\Delta\left(y_{5}^{2}, 4,4,8,2 t_{0}^{2}, y_{6}^{2}\right) \geq 0, y_{2}, y_{3} \in[2,2.168], y_{5} \in\left[2 t_{0}, 3.488\right] . \quad$ (893059266) $\operatorname{dih}+0.5\left(2.402-y_{4}\right)<\pi / 2$, if $y_{5} \geq 2 t_{0}, y_{2}, y_{3} \in[2,2.168]$.

(690646028)

\subsection{Group 4}

$\operatorname{dih}>1.78$, if $y_{4}=3.2, y_{1} \in\left[2 t_{0}, 2 \sqrt{2}\right], y_{2}+y_{3} \leq 4.6$.

(161665083)

\subsection{Group 5}

The following inequalities hold for flat quarters. In these inequalities the fourth edge is the diagonal. 


$$
\begin{array}{ll}
-\operatorname{dih}_{2} & +0.35 y_{2}-0.15 y_{1}-0.15 y_{3}+0.7022 y_{5}-0.17 y_{4}>-0.0123, \\
\operatorname{dih}_{2} & -0.13 y_{2}+0.631 y_{1}+0.31 y_{3}-0.58 y_{5}+0.413 y_{4}+0.025 y_{6}>2.63363, \\
-\operatorname{dih}_{1} & +0.714 y_{1}-0.221 y_{2}-0.221 y_{3}+0.92 y_{4}-0.221 y_{5}-0.221 y_{6}>0.3482, \\
\operatorname{dih}_{1} & -0.315 y_{1}+0.3972 y_{2}+0.3972 y_{3}- \\
& \quad 0.715 y_{4}+0.3972 y_{5}+0.3972 y_{6}>2.37095 \\
- \text { sol } & -0.187 y_{1}-0.187 y_{2}-0.187 y_{3}+0.1185 y_{4}+0.479 y_{5}+0.479 y_{6}>0.437235 \dagger, \\
\text { sol } & +0.488 y_{1}+0.488 y_{2}+0.488 y_{3}-0.334 y_{5}-0.334 y_{6}>2.244 \dagger \\
-\hat{\sigma} & -0.145 y_{1}-0.081 y_{2}-0.081 y_{3}-0.133 y_{5}-0.133 y_{6}>-1.17401 \\
-\hat{\sigma} & -0.12 y_{1}-0.081 y_{2}-0.081 y_{3}-0.113 y_{5}-0.113 y_{6}+0.029 y_{4}>-0.94903, \\
\hat{\sigma} & +0.153 y_{4}+0.153 y_{5}+0.153 y_{6}<1.05382 \\
\hat{\sigma} & +0.419351 \mathrm{sol}+0.19 y_{1}+0.19 y_{2}+0.19 y_{3}<1.449 \\
\hat{\sigma} & +0.419351 \mathrm{sol}<-0.01465+0.0436 y_{5}+0.0436 y_{6}+0.079431 \mathrm{dih}, \\
\hat{\sigma} & <0.0114 \\
\hat{\tau} & >1.019 p t
\end{array}
$$

(867513567)

\subsection{Group 6}

In a quadrilateral cluster, with a given edge $y_{4}$ as the diagonal, the other diagonal will be denoted $y_{4}^{\prime}$.

The following relations for upright quarters (scored by $\nu$ ) hold. (We use the inequalities of [5, Section 4] for upright quarters in quad clusters, which are scored by a different function.) In these inequalities the upright diagonal is the first edge. We include in this group, the inequalities $\left[6, \mathbf{A}_{2}\right],\left[6, \mathbf{A}_{3}\right]$ for upright quarters.

$$
\begin{array}{ll}
y_{1} & >2 t_{0}, \\
y_{1} & <2 \sqrt{2}, \\
\operatorname{dih}_{1} & -0.636 y_{1}+0.462 y_{2}+0.462 y_{3}-0.82 y_{4}+0.462 y_{5}+0.462 y_{6}>1.82419, \\
-\operatorname{dih}_{1} & +0.55 y_{1}-0.214 y_{2}-0.214 y_{3}+1.24 y_{4}-0.214 y_{5}-0.214 y_{6}>0.75281, \\
\operatorname{dih}_{2} & +0.4 y_{1}-0.15 y_{2}+0.09 y_{3}+0.631 y_{4}-0.57 y_{5}+0.23 y_{6}>2.5481, \\
-\operatorname{dih}_{2} & -0.454 y_{1}+0.34 y_{2}+0.154 y_{3}-0.346 y_{4}+0.805 y_{5}>-0.3429, \\
\operatorname{dih}_{3} & +0.4 y_{1}-0.15 y_{3}+0.09 y_{2}+0.631 y_{4}-0.57 y_{6}+0.23 y_{5}>2.5481, \\
-\operatorname{dih}_{3} & -0.454 y_{1}+0.34 y_{3}+0.154 y_{2}-0.346 y_{4}+0.805 y_{6}>-0.3429, \\
\operatorname{sol} & +0.065 y_{2}+0.065 y_{3}+0.061 y_{4}-0.115 y_{5}-0.115 y_{6}>0.2618, \\
-\operatorname{sol} & -0.293 y_{1}-0.03 y_{2}-0.03 y_{3}+0.12 y_{4}+0.325 y_{5}+0.325 y_{6}>0.2514, \\
-\nu & -0.0538 y_{2}-0.0538 y_{3}-0.083 y_{4}-0.0538 y_{5}-0.0538 y_{6}>-0.5995, \\
\nu & \leq 0, \quad(\operatorname{see}[2,3.13 .3] \text { and }[2,3.13 .4]) \\
\tau & -0.5945 p t>0 .
\end{array}
$$

(498839271)

$$
\begin{aligned}
& \nu \quad-4.10113 \operatorname{dih}_{1}<-4.3223 \\
& \nu \quad-0.80449 \operatorname{dih}_{1}<-0.9871 \\
& \nu \quad-0.70186 \operatorname{dih}_{1}<-0.8756 \\
& \nu \quad-0.24573 \operatorname{dih}_{1}<-0.3404 \\
& \nu \quad-0.00154 \operatorname{dih}_{1}<-0.0024 \\
& \nu \quad+0.07611 \operatorname{dih}_{1}<0.1196 .
\end{aligned}
$$

$\left(\left[6, \mathbf{A}_{2}\right]\right)$ 


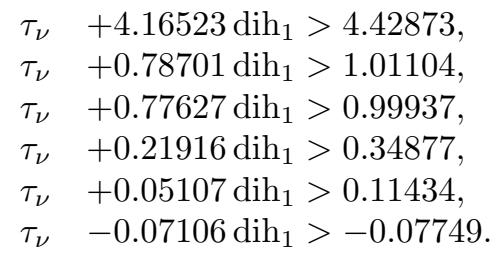

$\left(\left[6, \mathbf{A}_{3}\right]\right)$

\subsection{Group 7}

The following additional inequalities are known to hold if the upright diagonal has height at most 2.696. $\nu_{\Gamma}$ denotes the restriction of $\nu$ to a simplex of compression type.

$$
\begin{array}{rc}
y_{1} & <2.696 \\
\operatorname{dih}_{1} & -0.49 y_{1}+0.44 y_{2}+0.44 y_{3}-0.82 y_{4}+0.44 y_{5}+0.44 y_{6}>2.0421 \\
-\operatorname{dih}_{1} & +0.495 y_{1}-0.214 y_{2}-0.214 y_{3}+1.05 y_{4}-0.214 y_{5}-0.214 y_{6}>0.2282 \\
\operatorname{dih}_{2} & +0.38 y_{1}-0.15 y_{2}+0.09 y_{3}+0.54 y_{4}-0.57 y_{5}+0.24 y_{6}>2.3398 \\
-\operatorname{dih}_{2} & -0.375 y_{1}+0.33 y_{2}+0.11 y_{3}-0.36 y_{4}+0.72 y_{5}+0.034 y_{6}>-0.36135 \\
\text { sol } & +0.42 y_{1}+0.165 y_{2}+0.165 y_{3}-0.06 y_{4}-0.135 y_{5}-0.135 y_{6}>1.479 \\
-\operatorname{sol} & -0.265 y_{1}-0.06 y_{2}-0.06 y_{3}+0.124 y_{4}+0.296 y_{5}+0.296 y_{6}>0.0997 \\
-\nu & +0.112 y_{1}-0.142 y_{2}-0.142 y_{3}-0.16 y_{4}-0.074 y_{5}-0.074 y_{6}>-0.9029 \\
\nu & +0.07611 \operatorname{dih}_{1}<0.11 \\
\nu_{\Gamma} & -0.015 y_{1}-0.16\left(y_{2}+y_{3}+y_{4}\right)-0.0738\left(y_{5}+y_{6}\right)>-1.29285 \\
\tau_{\nu} & -0.07106 \operatorname{dih}_{1}>-0.06429 \\
\tau_{\nu} & >0.0414
\end{array}
$$

Remark 17.1. In connection with the Inequality (16), we occasionally use the stronger constant 0.2345 instead of 0.2282 . To justify this constant, we have checked using interval arithmetic that the bound 0.2345 holds if $y_{1} \leq 2.68$ or $y_{4} \leq 2.475$. Further interval calculations show that the anchored simplices can be erased if they share an upright diagonal with such a quarter.

\subsection{Group $8 \dagger$}

We give lower and upper bounds on dihedral angles. The domains that we list are not disjoint. In general we consider an edge as belonging to the most restrictive domain that the information of the following charts permit us to conclude that it lies in.

The following chart summarizes the bounds. The dihedral angle is computed along the first edge. The chart is divided into three sections. In the first, there is no upright diagonal. In the second, the upright diagonal is the first edge. In the third, the upright diagonal is the third edge. The bounds in the second section have been established in $\left[6, \mathbf{A}_{8}\right]$.

In the first group $y_{1}, y_{2}, y_{3} \in\left[2,2 t_{0}\right]$. In the third row, the dihedral bound 1.624 holds for $y_{6}$ on the larger interval $\left[2 t_{0}, 3.02\right]$. In the seventh row, the dihedral bound 1.507 holds for $y_{5}, y_{6}$ on the larger interval 
$\left[2 t_{0}, 3.02\right]$

$\begin{array}{ccccc}y_{5} & y_{6} & y_{4} & \mathrm{dih}_{\min } & \mathrm{dih}_{\max } \\ {\left[2,2 t_{0}\right]} & {\left[2,2 t_{0}\right]} & {\left[2 t_{0}, 2 \sqrt{2}\right]} & 1.153 & 2.28 \\ {\left[2,2 t_{0}\right]} & {\left[2,2 t_{0}\right]} & \geq 2 \sqrt{2} & 1.32 & 2 \pi \\ {\left[2,2 t_{0}\right]} & {\left[2 t_{0}, 2 \sqrt{2}\right]} & {\left[2,2 t_{0}\right]} & 0.633 & 1.624 \\ {\left[2,2 t_{0}\right]} & {\left[2 t_{0}, 2 \sqrt{2}\right]} & {\left[2 t_{0}, 2 \sqrt{2}\right]} & 1.033 & 1.929 \\ {\left[2,2 t_{0}\right]} & {\left[2 t_{0}, 2 \sqrt{2}\right]} & \geq 2 t_{0} & 1.033 & 2 \pi \\ {\left[2,2 t_{0}\right]} & {\left[2 t_{0}, 2 \sqrt{2}\right]} & \geq 2 \sqrt{2} & 1.259 & 2 \pi \\ {\left[2 t_{0}, 2 \sqrt{2}\right]} & {\left[2 t_{0}, 2 \sqrt{2}\right]} & {\left[2,2 t_{0}\right]} & 0.817 & 1.507 \\ {\left[2 t_{0}, 2 \sqrt{2}\right]} & {\left[2 t_{0}, 2 \sqrt{2}\right]} & {\left[2 t_{0}, 2 \sqrt{2}\right]} & 1.07 & 1.761 \\ {\left[2 t_{0}, 2 \sqrt{2}\right]} & {\left[2 t_{0}, 2 \sqrt{2}\right]} & \geq 2 t_{0} & 1.07 & 2 \pi \\ {\left[2 t_{0}, 2 \sqrt{2}\right]} & {\left[2 t_{0}, 2 \sqrt{2}\right]} & \geq 2 \sqrt{2} & 1.23 & 2 \pi\end{array}$

In the second group $y_{1} \in\left[2 t_{0}, 2 \sqrt{2}\right], y_{2}, y_{3} \in\left[2,2 t_{0}\right]$.

$\begin{array}{ccccc}y_{5} & y_{6} & y_{4} & \operatorname{dih}_{\min } & \operatorname{dih}_{\max } \\ {\left[2,2 t_{0}\right]} & {\left[2,2 t_{0}\right]} & {\left[2,2 t_{0}\right]} & 0.956 & 2.184 \\ {\left[2,2 t_{0}\right]} & {\left[2,2 t_{0}\right]} & {\left[2 t_{0}, 2 \sqrt{2}\right]} & 1.23 & \pi \\ {\left[2,2 t_{0}\right]} & {\left[2,2 t_{0}\right]} & \geq 2 t_{0} & 1.23 & \pi \\ {\left[2,2 t_{0}\right]} & {\left[2,2 t_{0}\right]} & \geq 2 \sqrt{2} & 1.416 & \pi\end{array}$

In the third group $y_{1}, y_{2} \in\left[2,2 t_{0}\right], y_{3} \in\left[2 t_{0}, 2 \sqrt{2}\right]$.

$\begin{array}{ccccc}y_{5} & y_{6} & y_{4} & \mathrm{dih}_{\min } & \mathrm{dih}_{\max } \\ {\left[2,2 t_{0}\right]} & {\left[2,2 t_{0}\right]} & {\left[2,2 t_{0}\right]} & 0.633 & 1.624 \\ {\left[2,2 t_{0}\right]} & {\left[2,2 t_{0}\right]} & \geq 2 t_{0} & 1.033 & 2 \pi \\ {\left[2,2 t_{0}\right]} & {\left[2 t_{0}, 2 \sqrt{2}\right]} & {\left[2,2 t_{0}\right]} & 0 & 1.381 \\ {\left[2,2 t_{0}\right]} & {\left[2 t_{0}, 2 \sqrt{2}\right]} & \geq 2 t_{0} & 0.777 & 2 \pi\end{array}$

(853728973)

\subsection{Group 9†}

(The verifications in this section that do not involve the score were verified by S. Ferguson.)

Depending on the lengths of the edges $y_{5}, y_{6}, y_{4}$, there are additional inequalities that hold. Generally these inequalities are for a fragment of a subregion. If $v_{2}, v_{1}, v_{3}$ are consecutive corners, we form a simplex $S\left(y_{1}, y_{2}, y_{3}, y_{4}, y_{5}, y_{6}\right)=\left(0, v_{1}, v_{2}, v_{3}\right)$. There is no score intrinsically associated with this simplex, unless the subregion is triangular. But we can state various useful bounds on the dihedral angles along the first edge $\left(0, v_{1}\right)$. A triple $(a, b, c)$ preceding the inequality gives bounds on the edges $\left(y_{5}, y_{6}, y_{4}\right)$, respectively. In this group of inequalities, $y_{1}, y_{2}, y_{3} \in\left[2,2 t_{0}\right]$. Set vor $_{x}=$ vor if the simplex has type $A$, and set 
$\operatorname{vor}_{x}=$ vor $_{0}$, otherwise.

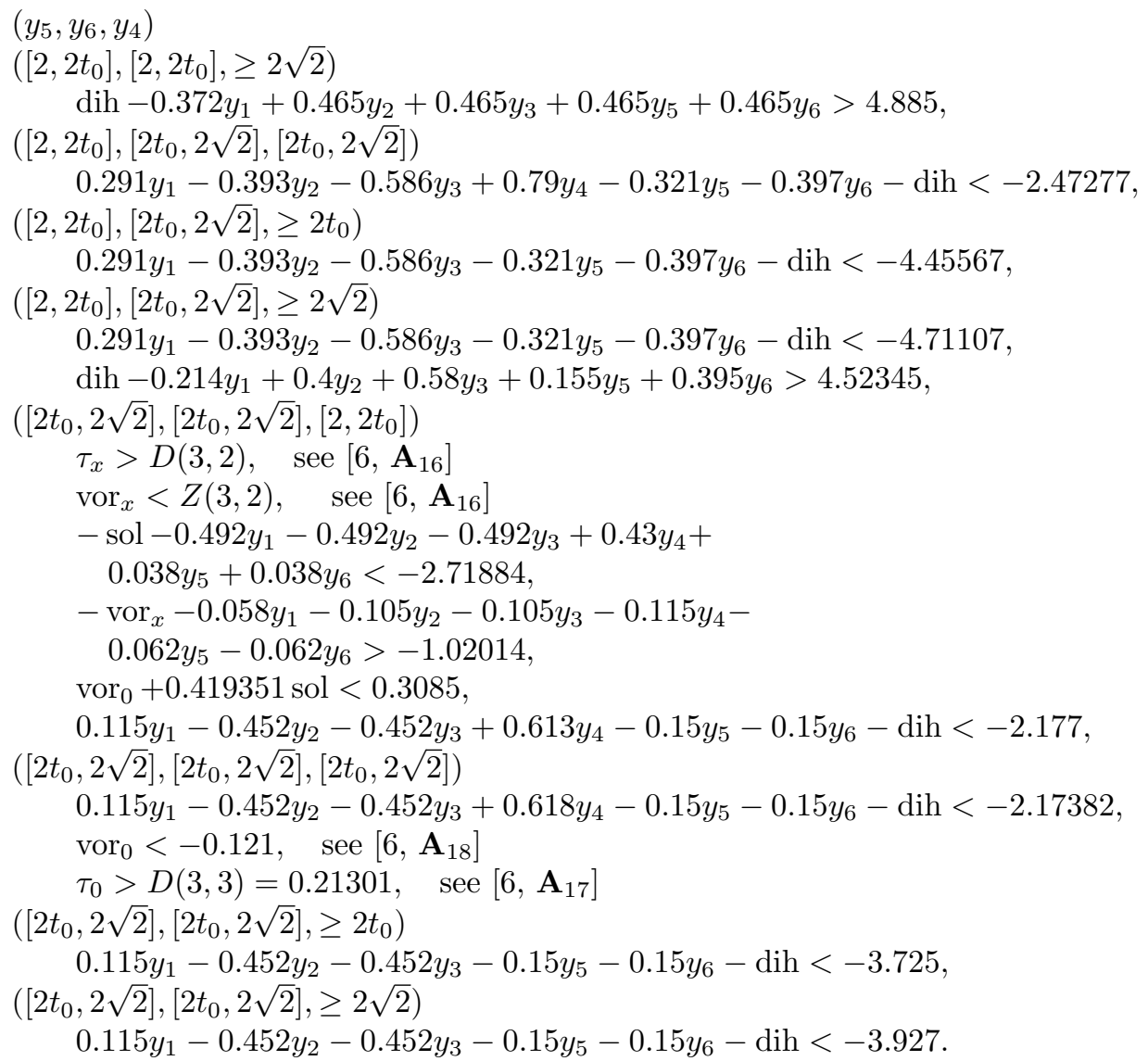

$(529738375)$

\section{$17.10 \quad$ Group 10}

In the next group $y_{1} \in\left[2 t_{0}, 2 \sqrt{2}\right], y_{2}, y_{3} \in\left[2,2 t_{0}\right]$. Set vor $_{x}=$ vor, if the simplex has type $C$ or $C^{\prime}$. Set $\operatorname{vor}_{x}=$ vor $_{0}$, otherwise. Other than this, retain the notation and conventions from Appendix 17.9.

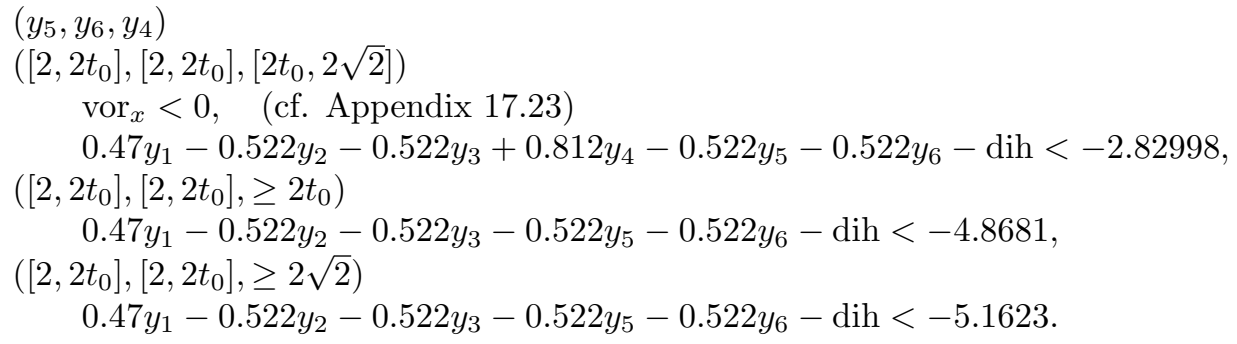

$(456320257)$ 


\section{$17.11 \quad$ Group 11}

In the next group $y_{1}, y_{2} \in\left[2,2 t_{0}\right], y_{3} \in\left[2 t_{0}, 2 \sqrt{2}\right]$. Otherwise, retain the notation and conventions of Appendix 17.9.

$$
\begin{aligned}
& \left(y_{5}, y_{6}, y_{4}\right) \\
& \left(\left[2,2 t_{0}\right],\left[2,2 t_{0}\right], \geq 2 t_{0}\right) \\
& \quad-0.4 y_{3}+0.15 y_{1}-0.09 y_{2}-0.631 y_{6}-0.23 y_{5}-\operatorname{dih}<-3.9788 \\
& \left(\left[2,2 t_{0}\right],\left[2 t_{0}, 2 \sqrt{2}\right],\left[2,2 t_{0}\right]\right) \\
& \quad 0.289 y_{1}-0.148 y_{2}-1.36 y_{3}+0.688 y_{4}-0.148 y_{5}-1.36 y_{6}-\operatorname{dih}<-6.3282, \\
& \left(\left[2,2 t_{0}\right],\left[2 t_{0}, 2 \sqrt{2}\right], \geq 2 t_{0}\right) \\
& \quad 0.289 y_{1}-0.148 y_{2}-0.723 y_{3}-0.148 y_{5}-0.723 y_{6}-\operatorname{dih}<-4.85746 .
\end{aligned}
$$

$(664959245)$

\subsection{Group 12}

1.

$$
\nu<-0.055 \text { and } \tau_{\nu}>0.092
$$

if $y_{1} \in[2.696,2 \sqrt{2}], y_{2}, y_{6} \in\left[2.45,2 t_{0}\right]$.

$(704795925)$

2.

$$
\hat{\sigma}<-0.039 \text { and } \hat{\tau}>0.094,
$$

if $y_{2} \in\left[2.45,2 t_{0}\right], y_{4} \in\left[2 t_{0}, 2 \sqrt{2}\right]$.

(332919646)

3.

$$
\text { vor }<-0.197 \text { and } \tau_{V}>0.239 \text {, }
$$

if $y_{1} \in[2.696,2 \sqrt{2}], y_{2} \in\left[2.45,2 t_{0}\right], y_{6} \in\left[2.45,2 t_{0}\right]$, and $y_{4} \in\left[2 t_{0}, 2 \sqrt{2}\right]$. (These simplices have type $S_{C}^{\prime}$ in the sense of $[6,2.10]$, and this justifies the use of the function vor.)

(335795137) 4 .

$$
\text { vor }_{0}<-0.089 \text { and } \tau_{0}>0.154
$$

if $y_{1} \in\left[2.45,2 t_{0}\right], y_{5}, y_{6} \in\left[2 t_{0}, 2 \sqrt{2}\right]$.

$(605071818 \dagger)$

5.

$$
\text { vor }_{0}<-0.089 \text { and } \tau_{0}>0.154 \text {, }
$$

if $y_{1} \in\left[2.45,2 t_{0}\right], y_{4}, y_{5} \in\left[2 t_{0}, 2 \sqrt{2}\right]$.

$(642806938 \dagger)$

\section{$17.13 \quad$ Group 13}

octavor $<$ octavor $_{0}-0.017$, if $y_{1} \in\left[2 t_{0}, 2.696\right]$, and $\eta_{126} \geq \sqrt{2}$.

$(104506452)$

$$
\operatorname{dih}>1.678, \text { if } y_{4} \geq 3.0, \text { and } y_{2}+y_{3}+y_{5}+y_{6} \leq 8.77,
$$

$(601083647)$

\subsection{Group 14}

$\Gamma<0.3138-0.157 y_{5}$, if $y_{5} \in[2,2.138], y_{4} \in\left[2 t_{0}, 2.6\right]$,

(543730647)

$\Gamma<-0.06$, if $y_{2} \in[2.121,2.145], y_{4} \in\left[2 t_{0}, 2 \sqrt{2}\right], y_{5} \in[2.22,2.238]$.

(163030624)

$\Gamma<10^{-6}+1.4-0.1 y_{1}-0.15\left(y_{2}+y_{3}+y_{5}+y_{6}\right)$, if $y_{4} \in[2,2 \sqrt{2}], y_{1}, y_{2}, y_{3} \in[2,2.2], y_{5}, y_{6} \in[2,2.35]$,

(181462710) 


\subsection{Group 15}

vor $<\operatorname{vor}_{0}$ if $y_{4} \in[2.7,2 \sqrt{2}], y_{1}, y_{2}, y_{3} \in[2,2.14]$.

(463544803)

vor $_{0}<-0.064$, if $y_{4} \in\left[2 t_{0}, 2.72\right], \eta_{456} \geq \sqrt{2}$.

(399326202)

vor $_{0}<1.0612-0.08\left(y_{1}+y_{2}+y_{3}\right)-0.142\left(y_{5}+y_{6}\right)$, if $y_{4} \in[2.7,2 \sqrt{2}]$.

(569240360)

vor $_{0}<-0.0713$, if $y_{4} \in[2.59,2.64], y_{5} \in\left[2.47,2 t_{0}\right], y_{6} \in[2.1,3.51]$.

(252231882)

vor $_{0}<-0.06$, if $y_{1}, y_{2}, y_{3} \in[2,2.13], y_{4} \in[2.7,2.74], \eta_{456} \geq \sqrt{2}$.

(472436131)

vor $_{0}<-0.058$, if $y_{4} \in\left[2 t_{0}, 2.747\right], \eta_{456} \geq \sqrt{2}$.

(913534858)

vor $_{0}<-0.0498$, if $y_{4} \in\left[2 t_{0}, 2.77\right], \eta_{456} \geq \sqrt{2}$.

(850226792)

\subsection{Group 16}

We assume that $y_{1}, y_{2}, y_{3} \leq 2.14$ in the following inequalities.

$-\Gamma-0.145 y_{1}-0.08\left(y_{2}+y_{3}\right)-0.133\left(y_{5}+y_{6}\right)>-1.146$, if $y_{4} \in\left[2 t_{0}, 2 \sqrt{2}\right]$.

(594246986)

$-\Gamma-0.145 y_{1}-0.081\left(y_{2}+y_{3}\right)-0.16\left(y_{5}+y_{6}\right)>-1.255$, if $y_{4} \in\left[2 t_{0}, 2 \sqrt{2}\right]$, and $y_{5}, y_{6} \in[2,2.3]$. (381970727)

$-\Gamma-0.03 y_{1}-0.03\left(y_{2}+y_{3}\right)-0.094\left(y_{5}+y_{6}\right)>-0.5361$. if $y_{4} \in\left[2 t_{0}, 2 \sqrt{2}\right], y_{5}+y_{6} \geq 4.3 . \quad$ (951798877)

$-\Gamma-0.03 y_{1}-0.03\left(y_{2}+y_{3}\right)-0.16\left(y_{5}+y_{6}\right)>-0.82-10^{-6}$. if $y_{4} \in\left[2 t_{0}, 2 \sqrt{2}\right], y_{5}+y_{6} \leq 4.3$. (923397705)

$\Gamma<-0.053$ if $y_{4} \in\left[2 t_{0}, 2 \sqrt{2}\right], y_{5} \geq 2.35$.

(312481617)

$\Gamma<-0.041$, if $y_{4} \in\left[2 t_{0}, 2 \sqrt{2}\right], y_{5} \in\left[2.25,2 t_{0}\right]$.

(292760488)

$\Gamma+0.419351 \mathrm{sol}<0.079431 \mathrm{dih}+0.0436\left(y_{5}+y_{6}\right)-0.0294$, if $\eta_{456} \leq \sqrt{2}, y_{4} \in\left[2 t_{0}, 2 \sqrt{2}\right]$.

(155008179)

$\Gamma<1.1457-0.1\left(y_{1}+y_{2}+y_{3}\right)-0.17 y_{5}-0.11 y_{6}$, if $y_{1}, y_{2}, y_{3} \in[2,2.13], y_{5} \in[2,2.1], y_{6} \in[2.27,2.43]$, $y_{4} \in\left[2 t_{0}, 2.67\right]$.

(819450002)

$1.69 y_{4}+y_{5}+y_{6}>9.0659$, if $y_{4} \in\left[2 t_{0}, 2.7\right]$ and $\eta_{456} \geq \sqrt{2}$.

(495568072)

$1.69 y_{4}+y_{5}+y_{6}>9.044$, if $y_{4} \in\left[2 t_{0}, 2.77\right]$ and $\eta_{456} \geq \sqrt{2}$.

(838887715)

$y_{5}+y_{6}>4.4$, if $\eta_{456} \geq \sqrt{2}$, and $y_{4} \leq 2.72$.

(794413343)

\subsection{Group 17}

In these inequalities $y_{5}, y_{6} \in\left[2 t_{0}, 2 \sqrt{2}\right]$ and $y_{4} \in\left[2,2 t_{0}\right]$. We distinguish three cases:

1. The simplex has type $A$, so that $y_{5}, y_{6} \in\left[2 t_{0}, 2.77\right]$, and the scoring is vor, the analytic Voronoi function.

2. $y_{6} \in[2.77,2 \sqrt{2}]$.

3. The edges have lengths $y_{5}, y_{6} \in\left[2 t_{0}, 2.77\right]$, and $\eta_{456} \geq \sqrt{2}$. In the last two cases, the scoring is by the truncated Voronoi function vor $_{0}$.

- vor $-0.058 y_{1}-0.08 y_{2}-0.08 y_{3}-0.16 y_{4}-0.21\left(y_{5}+y_{6}\right)>-1.7531$, if $y_{1}, y_{2}, y_{3} \leq 2.14$ and $y_{5}, y_{6} \in$ $\left[2 t_{0}, 2.77\right]$.

(378020227)

- vor $_{0}-0.058 y_{1}-0.1 y_{2}-0.1 y_{3}-0.165 y_{4}-0.115 y_{6}-0.12 y_{5}>-1.38875$, if $\eta_{456} \geq \sqrt{2}, y_{6} \in[2.77,2 \sqrt{2}]$, and $y_{1}, y_{2}, y_{3} \leq 2.14$.

(256893386)

$y_{4}+y_{5}+y_{6}>7.206$, if $y_{5}, y_{6} \in\left[2 t_{0}, 2.77\right]$ and $\eta_{456} \geq \sqrt{2}$.

(749955642)

- vor $_{0}-0.058 y_{1}-0.05 y_{2}-0.05 y_{3}-0.16 y_{4}-0.13 y_{6}-0.13 y_{5}>-1.24547$, if $y_{1}, y_{2}, y_{3} \leq 2.14, y_{5}, y_{6} \in$ $\left[2 t_{0}, 2.77\right]$, and $\eta_{456} \geq \sqrt{2}$.

(653849975)

vor $_{0}<-0.077$, if $y_{4} \in\left[2 t_{0}, 2 \sqrt{2}\right], y_{5} \in[2.77,2 \sqrt{2}]$.

(480930831) 
vor $+0.419351 \mathrm{sol}<0.289$, if $y_{4}, y_{5} \in\left[2 t_{0}, 2.77\right]$.

(271703736)

vor $_{0}<1.798-0.1\left(y_{1}+y_{2}+y_{3}\right)-0.19 y_{4}-0.17\left(y_{5}+y_{6}\right)$, if $y_{5}, y_{6} \in[2.7,2.77]$.

(900212351)

\subsection{Group 18}

The next inequality is used as an estimate when there is an upright diagonal enclosed over a simplex of the third type in Appendix 17.17.

vor $<-0.078 / 2, y_{1} \in\left[2 t_{0}, 2 \sqrt{2}\right], y_{4} \in\left[2 t_{0}, 2.6961\right]$ for simplices of type $C$.

$(455329491)$

If there is no enclosed upright diagonal, then we can sometimes use the following instead.

$\operatorname{vor}(S, \sqrt{2})<-0.078$, if $y_{5}, y_{6} \in\left[2 t_{0}, 2.6961\right], \eta_{456} \geq \sqrt{2}$.

(857241493)

\subsection{Group 19}

The interval calculations here show that the set of separated vertices (4) can be generalized to include opposite vertices of a quadrilateral unless the edge between those vertices forms a flat quarter. Consider a vertex of type $(3,1,1)$ with $a(3)=1.4 p t$. By the arguments in the text, we may assume that the dihedral angles of the exceptional regions at those vertices are at least 1.32 (see [6, 3.11.4]). Also, the three quasi-regular tetrahedra at the vertex squander at least $1.5 \mathrm{pt}$ by a linear programming bound, if the angle of the quad cluster is at least 1.55. Thus, we assume that the dihedral angles at opposite vertices of the quad cluster are at most 1.55. A linear program also gives $\tau+0.316$ dih $>0.3864$ for a quasi-regular tetrahedron.

If we give bounds of the form $\tau_{x}+0.316$ dih $>b$, for the part of the quad cluster around a vertex, where $\tau_{x}$ is the appropriate squander function, then we obtain

$$
\sum \tau_{x}>-0.316(2 \pi-1.32)+b+3(0.3864)
$$

for a lower bound on what is squandered. If the two opposite vertices give at least 2(1.4) $p t+0.1317$, then the inclusion of two opposite vertices in the separated set of vertices is justified. (Recall that $t_{4}=0.1317$.) The following inequalities give the desired result.

$\tau_{\mu}+0.316 \operatorname{dih}>0.5765$, if $\operatorname{dih} \leq 1.55, y_{4} \in\left[2 t_{0}, 2 \sqrt{2}\right]$.

(912536613)

$\tau_{0}+0.316$ dih $>0.5765$ if $\operatorname{dih} \leq 1.55, y_{4} \geq 2 \sqrt{2}$.

(640248153)

$\tau_{\nu}+0.316 \operatorname{dih}_{2}>0.2778$, if $y_{1} \in\left[2 t_{0}, 2 \sqrt{2}\right]$,

(594902677)

\subsection{Group 20†}

If the circumradius of a quasi-regular tetrahedron is $\geq 1.41$, then by [3, Section 9.17], $\tau>1.8 p t$, and many of the inequalities hold (without further interval arithmetic calculations).

In Sections 17.20 and 17.21 , let $S_{1}, \ldots, S_{5}$ be 5 simplices arranged around a common edge $(0, v)$, with $|v| \in\left[2,2 t_{0}\right]$. Let $y_{i}\left(S_{j}\right)$ be the edges, with $y_{1}\left(S_{j}\right)=|v|$ for all $j, y_{3}\left(S_{j}\right)=y_{2}\left(S_{j+1}\right)$, and $y_{5}\left(S_{j}\right)=$ $y_{6}\left(S_{j+1}\right)$. where the subscripts $j$ are extended modulo 5. In Sections 17.20 and $17.21, \sum \operatorname{dih}\left(S_{j}\right) \leq 2 \pi$. Set $\pi_{F}=2 \xi_{V}+\xi_{\Gamma}$ if $\hat{\sigma}=\operatorname{vor}_{0}$ in the cases $\left(y_{4} \geq 2.6, y_{1} \geq 2.2\right)$ and $\left(y_{4} \geq 2.7\right)$. Set $\pi_{F}=0$, otherwise.

$$
\begin{array}{lr}
\tau\left(S_{1}\right)+\tau\left(S_{2}\right)+\tau\left(S_{4}\right)>1.4 p t, \text { if } y_{4}\left(S_{3}\right), y_{4}\left(S_{5}\right) \geq 2 \sqrt{2} . & \text { (551665569) } \\
\tau\left(S_{1}\right)+\tau\left(S_{2}\right)+\tau\left(S_{3}\right)>1.4 p t, \text { if } y_{4}\left(S_{4}\right), y_{4}\left(S_{5}\right) \geq 2 \sqrt{2} . & (67562926) \\
\tau\left(S_{1}\right)+\tau\left(S_{2}\right)+\left(\hat{\tau}\left(S_{3}\right)-\pi_{F}\right)+\tau\left(S_{4}\right)>1.4 p t+D(3,1), \text { if } y_{4}\left(S_{3}\right) \in\left[2 t_{0}, 2 \sqrt{2}\right], y_{4}\left(S_{5}\right) \geq 2 t_{0}, & \operatorname{dih}\left(S_{5}\right)>1.32, \\
\tau\left(S_{1}\right)+\tau\left(S_{2}\right)+\tau\left(S_{3}\right)+\left(\hat{\tau}\left(S_{4}\right)-\pi_{F}\right)>1.4 p t+D(3,1), \text { if } y_{4}\left(S_{4}\right) \in\left[2 t_{0}, 2 \sqrt{2}\right], y_{4}\left(S_{5}\right) \geq 2 t_{0}, \operatorname{dih}\left(S_{5}\right)>1.32 .
\end{array}
$$




\subsection{Group $21 \dagger$}

As in Section 17.20, the quasi-regular tetrahedra are generally compression scored. Define $\pi_{F}$ as in Section 17.20. The constraint $\sum_{(5)} \operatorname{dih}\left(S_{j}\right)=2 \pi$ is assumed.

$\tau\left(S_{1}\right)+\tau\left(S_{2}\right)+\tau\left(S_{3}\right)+\tau\left(S_{4}\right)>1.5 p t$, if $y_{4}\left(S_{5}\right) \geq 2 \sqrt{2}$.

(325738864)

$\tau\left(S_{1}\right)+\tau\left(S_{2}\right)+\tau\left(S_{3}\right)+\tau\left(S_{4}\right)+\left(\hat{\tau}\left(S_{5}\right)-\pi_{F}\right)>1.5 p t+D(3,1)$, if $y_{4}\left(S_{5}\right) \in\left[2 t_{0}, 2 \sqrt{2}\right]$.

(314974315)

\subsection{Group 22}

If there are four quasi-regular tetrahedra $\left\{S_{1}, \ldots, S_{4}\right\}$ at the central vertex $v$ of the flat quarter $Q$, and if there are only five standard regions at $v$, then

$$
\hat{\sigma}(Q)+\sum_{(4)} \sigma\left(S_{i}\right)<0.114
$$

(867359387)

\section{$17.23 \quad$ Group 23}

Let $\operatorname{vor}_{x}=$ vor for simplices of types $C$ and $C^{\prime}$. Let vor $_{x}=$ vor, otherwise.

$$
\operatorname{vor}_{x}< \begin{cases}0, & y_{1} \in\left[2 t_{0}, 2 \sqrt{2}\right] \\ -0.05, & y_{1} \in\left[2 t_{0}, 2.696\right], \\ -0.119, & y_{1} \in\left[2 t_{0}, 2.696\right], \quad \eta_{126} \geq \sqrt{2},\end{cases}
$$

if $y_{4} \in\left[2 t_{0}, 2 \sqrt{2}\right]$. The bound of 0 is established in Appendix 17.10. (Even when simplex has type $C$, the bound -0.05 is based on the upper bound vor $_{0}$.)

(365179082)

\subsection{Group 24}

$$
\sigma_{R}(D)< \begin{cases}0, & y_{1} \in\left[2 t_{0}, 2 \sqrt{2}\right], \\ -0.043, & y_{1} \in\left[2 t_{0}, 2.696\right]\end{cases}
$$

for quad regions $R$ constructed from an anchored simplex $S$ and adjacent special simplex $S^{\prime}$. Assume that $y_{4}(S)=y_{4}\left(S^{\prime}\right) \in[2 \sqrt{2}, 3.2]$, and that the other edges have lengths in $\left[2,2 t_{0}\right]$. The bound 0 is found in [2, Lemma 3.13]. The bound -0.043 is obtained from deformations, reducing the inequality to the following interval calculations.

$$
\begin{aligned}
& \text { vor }_{0}<-0.043 / 2 \text {, if } y_{6}=2 t_{0}, y_{1} \in\left[2 t_{0}, 2.696\right] \text {. } \\
& \operatorname{vor}_{0}(S)+\operatorname{vor}_{0}\left(S\left(2, y_{2}, y_{3}, y_{4}, 2,2\right)\right)<-0.043 \text {, if } y_{1} \in\left[2 t_{0}, 2.696\right], y_{4} \in[2 \sqrt{2}, 3.2], y_{4}^{\prime} \geq 2 t_{0} . \text { (820900672†) } \\
& \operatorname{vor}_{0}(S)+\operatorname{vor}_{0}\left(S\left(2 t_{0}, y_{2}, y_{3}, y_{4}, 2,2\right)\right)<-0.043 \text {, if } y_{1} \in\left[2 t_{0}, 2.696\right], y_{4} \in[2 \sqrt{2}, 3.2], y_{4}^{\prime} \geq 2 t_{0} \text {. } \\
& \operatorname{vor}_{0}\left(S^{\prime}\right)<-0.033, \text { if } \operatorname{dih}\left(S^{\prime}\right) \leq 1.8, y_{1} \in[2,2.12], y_{4} \in[2 \sqrt{2}, 3.2] \text {, } \\
& \operatorname{vor}_{0}(S) \quad<-0.058, \text { if } \operatorname{dih}(S) \leq 2.5, y_{1} \in\left[2 t_{0}, 2.696\right], y_{4} \in[2 \sqrt{2}, 3.2] \text {, } \\
& \operatorname{vor}_{0}(S) \quad<-0.073 \text {, if } \eta_{126} \geq \sqrt{2}, y_{1} \in\left[2 t_{0}, 2.696\right], y_{4} \in[2 \sqrt{2}, 3.2] . \dagger
\end{aligned}
$$$$
(961078136 \dagger)
$$ 
These combine to give

$$
\operatorname{vor}_{0}(S)+\operatorname{vor}_{0}\left(S^{\prime}\right)< \begin{cases}-0.091, & \text { or } \\ -0.106, & \end{cases}
$$

for the combination of special simplex and anchored simplex under the stated conditions.

\subsection{Group 25 (pentagons)}

There are a few inequalities that arise for pentagonal regions.

Proposition 17.2. If the pentagonal region has no flat quarters and no upright quarters, the subregion $F$ is a pentagon. It satisfies

$$
\begin{array}{ll}
\text { vor }_{0} & <-0.128, \\
\tau_{0} & >0.36925 .
\end{array}
$$

Proof. The proof is by deformations and interval calculations. If a deformation produces a new flat quarter, then the result follows from $\left[6, \mathbf{A}_{13}\right]$ and Inequality 18.2 . So we may assume that all diagonals remain at least $2 \sqrt{2}$. If all diagonals remain at least 3.2 , the result follows from the tcc-bound on the pentagon [6, Section 5.5]. Thus, we assume that some diagonal is at most 3.2. We deform the cluster into the form

$$
\left(a_{1}, 2, a_{2}, 2, a_{3}, 2, a_{4}, 2, a_{5}, 2\right), \quad\left|v_{i}\right|=a_{i} \in\left\{2,2 t_{0}\right\} .
$$

Assume that $\left|v_{1}-v_{3}\right| \leq 3.2$. If $\max \left(a_{1}, a_{3}\right)=2 t_{0}$, the result follows from $\left[6, \mathbf{A}_{13}\right]$ and Section 18.2 , Equations 31 and 32 .

Assume $a_{1}=a_{3}=2$. There is a diagonal of the quadrilateral of length at most 3.23 because

$$
\Delta\left(3.23^{2}, 4,4,3.23^{2}, 4,3.2^{2}\right)<0 .
$$

The result now follows from the following interval arithmetic calculations.

(These inequalities are closely related to $\left[6, \mathbf{A}_{21}\right]$.)

$\operatorname{vor}_{0}\left(S\left(2,2,2, y_{4}, 2,2\right)\right)+\operatorname{vor}_{0}\left(S\left(2,2,2, y_{4}^{\prime}, 2,2\right)\right)+\operatorname{vor}_{0}\left(S\left(2,2,2, y_{4}, y_{4}^{\prime}, 2\right)\right)<-0.128$, if $y_{4} \in[2 \sqrt{2}, 3.2]$, $y_{4}^{\prime} \in[2 \sqrt{2}, 3.23]$.

$(587781327 \dagger)$

$\tau_{0}\left(S\left(2,2,2, y_{4}, 2,2\right)\right)+\tau_{0}\left(S\left(2,2,2, y_{4}^{\prime}, 2,2\right)\right)+\tau_{0}\left(S\left(2,2,2, y_{4}, y_{4}^{\prime}, 2\right)\right)>0.36925$, if $y_{4} \in[2 \sqrt{2}, 3.2], y_{4}^{\prime} \in$ $[2 \sqrt{2}, 3.23]$.

(807067544†)

$\tau_{0}\left(2,2, y_{3}, y_{4}, 2,2\right)<\tau_{0}\left(2 t_{0}, 2, y_{3}, y_{4}, 2,2\right)$, if $y_{4} \in[2 \sqrt{2}, 3.06]$.

(986970370†)

$\operatorname{vor}_{0}\left(2,2, y_{3}, y_{4}, 2,2\right)>\operatorname{vor}_{0}\left(2 t_{0}, 2, y_{3}, y_{4}, 2,2\right)$, if $y_{4} \in[2 \sqrt{2}, 3.06]$.

(677910379†)

vor $_{0}<-0.128$, if $y_{1}=y_{2}=y_{4}=2, y_{5}, y_{6} \in[3.06,3.23]$.

(276168273)

$\tau_{0}>0.36925$, if $y_{1}=y_{2}=y_{3}=y_{4}=2, y_{5}, y_{6} \in[3.06,3.23]$.

(411203982)

$\tau_{0}>0.31$, if $y_{1}=y_{2}=y_{4}=2, y_{3}=2 t_{0}, y_{5}, y_{6} \in[3.06,3.23]$.

(860823724)

vor $_{0}<-0.137-\left(y_{5}-2 \sqrt{2}\right) 0.14$, if $y_{1}=y_{4}=2, y_{5} \in[2 \sqrt{2}, 3.23], y_{6} \in[3.06,3.23]$.

(353116955)

$\tau_{0}>0.31+\left(y_{5}-2 \sqrt{2}\right) 0.14$, if $y_{1}=y_{4}=2, y_{5} \in[2 \sqrt{2}, 3.23], y_{6} \in[3.105,3.23]$

(943315982)

$\tau_{0}>0.31+\left(y_{5}-2 \sqrt{2}\right) 0.14+\left(y_{6}-3.105\right) 0.19$, if $y_{1}=y_{4}=2, y_{5} \in[2 \sqrt{2}, 3.23], y_{6} \in[3.06,3.105]$.

(941799628)

vor $_{0}<0.009+\left(y_{5}-2 \sqrt{2}\right) 0.14$, if $y_{1}=y_{4}=2, y_{5} \in[2 \sqrt{2}, 3.23], y_{2}=y_{6}=2$.

(674284283)

$\tau_{0}>0.05925+\left(y_{5}-2 \sqrt{2}\right) 0.14$, if $y_{1}=y_{4}=2, y_{5} \in[2 \sqrt{2}, 3.23], y_{2}=y_{6}=2$.

(775220784)

$\tau_{0}>0.05925$, if $y_{3}=2 t_{0}, y_{5}=y_{6}=2, y_{4} \in[2 \sqrt{2}, 3.23]$.

(286076305)

$\tau_{0}>-\left(y_{4}-3.105\right) 0.19$, if $y_{1}=2 t_{0}, y_{2}=y_{3}=y_{5}=y_{6}=2, y_{4} \in[3.06,3.105]$.

(589319960) 


\subsection{Group $26 \dagger$}

Let $Q$ be a quadrilateral region with parameters

$$
\left(a_{1}, 2 t_{0}, a_{2}, 2, a_{3}, 2, a_{4}, 2 t_{0}\right), \quad a_{i} \in\left\{2,2 t_{0}\right\} .
$$

Assume that $\left|v_{2}-v_{4}\right| \in[2 \sqrt{2}, 3.2],\left|v_{1}-v_{3}\right| \in[3.2,3.46]$. Note that

$$
\Delta\left(4,4,8,2 t_{0}^{2}, 2 t_{0}^{2}, 3.46^{2}\right)<0 .
$$

$\operatorname{vor}_{0}(Q)<-0.168$.

(302085207)

$\tau_{0}(Q)>0.352$.

(411491283)

\section{$17.27 \quad$ Group 27}

Consider a pentagonal region. If the pentagonal region has one flat quarter and no upright quarters, there is a quadrilateral region $F$. It satisfies

$$
\begin{aligned}
& \operatorname{vor}_{0}<-0.075, \\
& \tau_{0}>0.176 .
\end{aligned}
$$

Break the cluster into two simplices $S=S\left(y_{1}, \ldots, y_{6}\right), S^{\prime}=S\left(y_{1}^{\prime}, y_{2}, y_{3}, y_{4}, y_{5}^{\prime}, y_{6}^{\prime}\right)$, by drawing a diagonal of length $y_{4}$. Assume that the edge $y_{5}^{\prime} \in\left[2 t_{0}, 2 \sqrt{2}\right]$. Let $y_{4}^{\prime}$ be the length of the diagonal that crosses $y_{4}$.

$$
\begin{aligned}
\operatorname{vor}_{0}< & 2.1327-0.1 y_{1}-0.15 y_{2}-0.08 y_{3}-0.15 y_{5} \\
& -0.15 y_{6}-0.1 y_{1}^{\prime}-0.17 y_{5}^{\prime}-0.16 y_{6}^{\prime}, \\
& \text { if } \operatorname{dih}(S)<1.9, \operatorname{dih}\left(S^{\prime}\right)<2.0, y_{1} \in[2,2.2], y_{4} \geq 2 \sqrt{2}, \\
\operatorname{vor}_{0}< & 2.02644-0.1 y_{1}-0.14\left(y_{2}+y_{3}\right)-0.15\left(y_{5}+y_{6}\right)-0.1 y_{1}^{\prime}-0.12\left(y_{5}^{\prime}+y_{6}^{\prime}\right), \\
& \text { if } y_{1} \in[2,2.08], \quad y_{4} \leq 3 \\
\text { vor }_{0} \quad+ & 0.419351 \mathrm{sol}<0.4542+0.0238\left(y_{5}+y_{6}+y_{6}^{\prime}\right), \\
& \text { if } y_{4}, y_{4}^{\prime} \geq 2 \sqrt{2} .
\end{aligned}
$$

The inequalities above are verified in smaller pieces:

vor $_{0}<1.01-0.1 y_{1}-0.05 y_{2}-0.05 y_{3}-0.15 y_{5}-0.15 y_{6}$, if dih $\leq 1.9, y_{4} \geq 2 \sqrt{2}$, and $y_{1} \leq 2.2$. (131574415) vor $_{0}<1.1227-0.1 y_{1}-0.1 y_{2}-0.03 y_{3}-0.17 y_{5}-0.16 y_{6}$, if $\operatorname{dih} \leq 2, y_{4} \geq 2 \sqrt{2}, y_{5} \in\left[2 t_{0}, 2 \sqrt{2}\right]$, and $y_{2}+y_{3} \leq 4.67$.

vor $_{0}<1.0159-0.1 y_{1}-0.08\left(y_{2}+y_{3}\right)+0.04 y_{4}-0.15\left(y_{5}+y_{6}\right)$, if $y_{4} \in[2 \sqrt{2}, 3], y_{1} \in[2,2.08]$. (223261160) vor $_{0}<1.01054-0.1 y_{1}-0.06\left(y_{2}+y_{3}\right)-0.04 y_{4}-0.12\left(y_{5}+y_{6}\right)$, if $y_{4} \in[2 \sqrt{2}, 3], y_{5} \in\left[2 t_{0}, 2 \sqrt{2}\right]$. (135018647) Let $Q$ be the quadrilateral subcluster expressed as a union of two simplices $S$ and $S^{\prime}$, as above

$$
\operatorname{vor}_{0}(Q)+0.419351 \operatorname{sol}(Q)<0.4542+0.0238\left(y_{5}+y_{6}+y_{6}^{\prime}\right),
$$

if $y_{5}^{\prime} \in\left[2 t_{0}, 2 \sqrt{2}\right]$ and both diagonals have length $\geq 2 \sqrt{2}$.

(559676877)

\subsection{Group 28†}

If $v$ is a vertex of an exceptional cluster and there are exactly 4 quasi-regular tetrahedra along $(0, v)$, then there are 5 vertices $v_{1}, \ldots, v_{5}$ adjacent to $v$. We have

$$
\sum_{(5)}\left(\left|v-v_{i}\right|+\left|v_{i}\right|\right)>20.42 .
$$

(615073260) 
If $v_{1}$ and $v_{5}$ are the two vertices on an exceptional cluster, and if the additional hypothesis $\left|v_{1}-v_{5}\right| \geq 2 \sqrt{2}$ holds, then

$$
\sum_{(5)}\left(\left|v-v_{i}\right|+\left|v_{i}\right|\right)>20.76
$$

(844430737)

\section{$17.29 \quad$ Group 29}

$$
\text { vor }_{0}<-0.136 \text { and } \tau_{0}>0.224 \text {, }
$$

for a combination of anchored simplex $S$ and special simplex $S^{\prime}$, with $y_{1}(S) \in[2.696,2 \sqrt{2}], y_{2}(S), y_{6}(S) \in$ $\left[2.45,2 t_{0}\right], y_{4}(S) \in[2 \sqrt{2}, 3.2]$, and with cross-diagonal at least $2 t_{0}$. This inequality can be verified by proving the following inequalities in lower dimension. In the first four $y_{1} \in[2.696,2 \sqrt{2}], y_{2}, y_{6} \in\left[2.45,2 t_{0}\right]$, $y_{4} \in[2 \sqrt{2}, 3.2]$, and $y_{4}^{\prime} \geq 2 t_{0}$ (the cross-diagonal).

$$
\begin{aligned}
& \operatorname{vor}_{0}\left(S\left(y_{1}, \ldots, y_{6}\right)\right)+\operatorname{vor}_{0}\left(S\left(2, y_{2}, y_{3}, y_{4}, 2,2\right)\right)<-0.136 \\
& \operatorname{vor}_{0}\left(S\left(y_{1}, \ldots, y_{6}\right)\right)+\operatorname{vor}_{0}\left(S\left(2 t_{0}, y_{2}, y_{3}, y_{4}, 2,2\right)\right)<-0.136 \\
& \tau_{0}\left(S\left(y_{1}, \ldots, y_{6}\right)\right)+\tau_{0}\left(S\left(2, y_{2}, y_{3}, y_{4}, 2,2\right)\right)>0.224 \\
& \tau_{0}\left(S\left(y_{1}, \ldots, y_{6}\right)\right)+\tau_{0}\left(S\left(2 t_{0}, y_{2}, y_{3}, y_{4}, 2,2\right)\right)>0.224 \\
& \operatorname{vor}_{0}\left(S\left(y_{1}, \ldots, y_{6}\right)\right)<-0.125, \text { if } y_{1} \in[2.696,2 \sqrt{2}], y_{2}, y_{6} \in\left[2.45,2 t_{0}\right], y_{5}=2 t_{0} . \\
& \operatorname{vor}_{0}\left(S\left(y_{1}, \ldots, y_{6}\right)\right)<0.011, \text { if } y_{1} \in[2.696,2 \sqrt{2}], y_{5}=2 t_{0} . \\
& \tau_{0}\left(S\left(y_{1}, \ldots, y_{6}\right)\right)>0.17, \text { if } y_{1} \in[2.696,2 \sqrt{2}], y_{2}, y_{6} \in\left[2.45,2 t_{0}\right], y_{5}=2 t_{0} . \\
& \tau_{0}\left(S\left(y_{1}, \ldots, y_{6}\right)\right)>0.054, \text { if } y_{1} \in[2.696,2 \sqrt{2}], y_{5}=2 t_{0} .
\end{aligned}
$$

\section{$17.30 \quad$ Group 30}

$$
\text { vor }_{0}<-0.24 \text { and } \tau_{0}>0.346 \text {, }
$$

for an anchored simplex $S$ and simplex $S^{\prime}$ with edge parameters $(3,2)$ in a hexagonal cluster, with $y_{2}(S)=y_{2}\left(S^{\prime}\right), y_{3}(S)=y_{3}\left(S^{\prime}\right), y_{4}(S)=y_{4}\left(S^{\prime}\right), y_{1}(S) \in[2.696,2 \sqrt{2}], y_{4}(S) \in[2 \sqrt{2}, 3.2], y_{2}(S), y_{6}(S) \in$ $\left[2.45,2 t_{0}\right]$, and

$$
\max \left(y_{5}\left(S^{\prime}\right), y_{6}\left(S^{\prime}\right)\right) \in\left[2 t_{0}, 2 \sqrt{2}\right], \quad \min \left(y_{5}\left(S^{\prime}\right), y_{6}\left(S^{\prime}\right)\right) \in\left[2,2 t_{0}\right] .
$$

This breaks into separate interval calculations for $S$ and $S^{\prime}$.

This inequality results from the following four inequalities:

$\operatorname{vor}_{0}(S)<-0.126$ and $\tau_{0}(S)>0.16$

$(369386367 \dagger)$

$\operatorname{vor}_{0}\left(S^{\prime}\right)<-0.114$ and $\tau_{0}\left(S^{\prime}\right)>0.186$ (There are two cases for each, depending on which of $y_{5}, y_{6}$ is longer.)

(724943459†)

\subsection{Group 31}

$$
\text { vor }_{0}<-0.149 \text { and } \tau_{0}>0.281 \text {, }
$$

for a quadrilateral subcluster with both diagonals $\geq 2 \sqrt{2}$, and parameters

$$
\left(a_{1}, b_{1}, a_{2}, b_{2}, a_{3}, b_{3}, a_{4}, b_{4}\right),
$$

with $a_{4} \in\left[2.45,2 t_{0}\right]$, and $b_{4} \in\left[2 t_{0}, 2 \sqrt{2}\right]$.

$(836331201 \dagger)$ 


\section{$17.32 \quad$ Group 32}

$$
\text { vor }_{0}<-0.254 \text { and } \tau_{0}>0.42625
$$

for a combination of anchored simplex $S$ and quadrilateral cluster $Q$. It is assumed that $y_{1}(S) \in$ $[2.696,2 \sqrt{2}], y_{2}(S), y_{6}(S) \in\left[2.45,2 t_{0}\right]$. The adjacent quadrilateral subcluster is assumed to have both diagonals $\geq 2 \sqrt{2}$, and parameters

$$
\left(a_{1}, b_{1}, a_{2}, b_{2}, a_{3}, b_{3}, a_{4}, b_{4}\right)
$$

with $b_{4} \in[2 \sqrt{2}, 3.2]$. The verification of this inequality reduces to separate inequalities for the anchored simplex and quadrilateral subcluster. For the anchored simplex we use the bounds $\operatorname{vor}_{0}\left(S^{\prime}\right)<-0.126$, $\tau_{0}\left(S^{\prime}\right)>0.16$ that have already been established above. We then show that the quad cluster satisfies

vor $_{0}<-0.128$ and $\tau_{0}>0.26625$.

$(327474205 \dagger)$

For this, use deformations to reduce either to the case where the diagonal is $2 \sqrt{2}$, or to the case where $b_{1}=b_{2}=b_{3}=2, a_{2}, a_{3} \in\left\{2,2 t_{0}\right\}$. When the diagonal is $2 \sqrt{2}$, the flat quarter can be scored by [6, $\left.\mathbf{A}_{13}\right]$ : $\left(\operatorname{vor}_{0}<0.009, \tau_{0}>0.05925\right)$. (There are two cases depending on which direction the diagonal of length $\sqrt{2}$ runs.) 


\section{Appendix Hexagonal In- equalities}

There are a number of inequalities that have been particularly designed for standard regions that are hexagons. This appendix describes those inequalities. They are generally inequalities involving more than six variables, and because of current technological limitations on interval arithmetic, we were not able to prove these inequalities directly with interval arithmetic.

Instead we give various lemmas that deduce the inequalities from inequalities in a smaller number of variables (small enough to prove by interval arithmetic.)

\subsection{Statement of results}

There are a number of inequalities that hold in special situations when there is a hexagonal region. After stating all of them, we will turn to the proofs.

1. If there are no flat quarters and no upright quarters (so that there is a single subregion $F$ ), then

$$
\begin{array}{rr}
\text { vor }_{0} & <-0.212 \\
\tau_{0} & >0.54525 .
\end{array}
$$

2. If there is one flat quarter and no upright quarters, there is a pentagonal subregion $F$. It satisfies

$$
\begin{array}{ll}
\text { vor }_{0} & <-0.221 \\
\tau_{0} & >0.486 .
\end{array}
$$

3. If there are two flat quarters and no upright quarters, there is a quadrilateral subregion $F$. It satisfies

$$
\begin{aligned}
& \operatorname{vor}_{0} \quad<-0.168, \\
& \tau_{0}>0.352 .
\end{aligned}
$$

These are twice the constants appearing in 11;

4. If there is an edge of length between $2 t_{0}$ and $2 \sqrt{2}$ running between two opposite corners of the hexagonal cluster, and if there are no flat or upright quarters on one side, leaving a quadrilateral region $F$, then $F$ satisfies

$$
\begin{aligned}
& \text { vor }_{0}<-0.075, \\
& \tau_{0}>0.176 .
\end{aligned}
$$

5. If the hexagonal cluster has an upright diagonal with context $(4,2)$, and if there are no flat quarters (Figure 20, then the hexagonal cluster $R$ satisfies

$$
\begin{aligned}
& \sigma_{R}<-0.297, \\
& \tau_{R}>0.504 .
\end{aligned}
$$

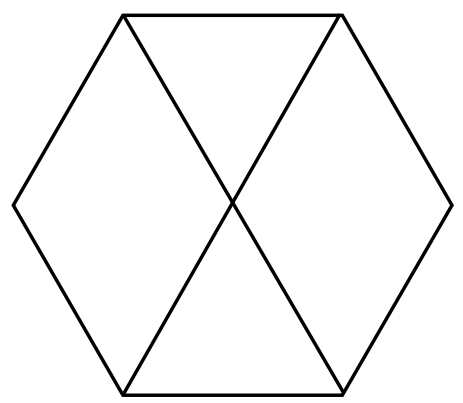

Figure 20: A hexagonal cluster with context $(4,2)$.

6. If the hexagonal cluster has an upright diagonal with context $(4,2)$, and if there is one unmasked flat quarter (Figure 21, let $\{F\}$ be the set of four subregions around the upright diagonal. (That is, take all subregions except for the flat quarter.) In the following inequality and Inequality 7 , let $\sigma_{R}^{+}$be defined as $\sigma_{R}$ on quarters, and vor $_{x}$ on other anchored simplices. $\tau_{R}^{+}$is the adapted squander function.

$$
\begin{aligned}
& \sum_{(4)} \sigma_{R}^{+}<-0.253, \\
& \sum_{(4)} \tau_{R}^{+}>0.4686 .
\end{aligned}
$$

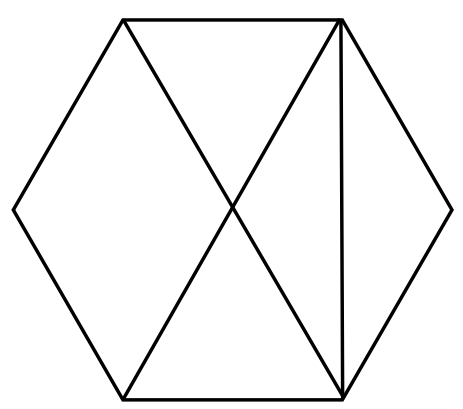

Figure 21: A hexagonal cluster with context $(4,2)$.

7. If the hexagonal cluster has an upright diagonal with context $(4,2)$, and if there are two unmasked flat quarters (Figure 22, let $\{F\}$ be the set of four subregions around the upright 
diagonal. (That is, take all subregions except for the flat quarters.)

$$
\begin{aligned}
& \sum_{(4)} \sigma_{R}^{+} \quad<-0.2, \\
& \sum_{(4)} \tau_{R}^{+}>0.3992 .
\end{aligned}
$$

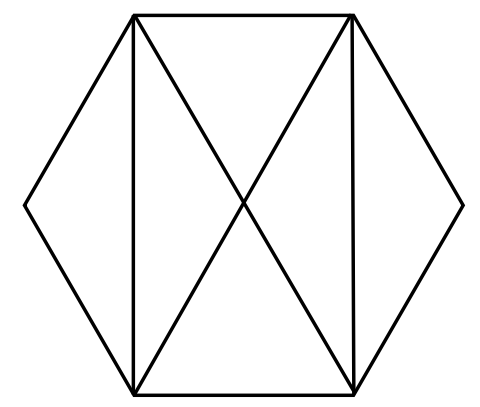

Figure 22: A hexagonal cluster with context $(4,2)$.

8. If the hexagonal cluster has an upright diagonal in context $(4,1)$, and if there are no flat quarters, let $\{F\}$ be the set of four subregions around the upright diagonal. Assume that the edge opposite the upright diagonal on the anchored simplex has length at least $2 \sqrt{2}$. (See Figure 23.)

$$
\begin{array}{ll}
\operatorname{vor}_{0, R}(D)+\sum_{(3)} \sigma(Q) & <-0.2187 \\
\tau_{0, R}(D)+\sum_{(3)} \tau(Q) & >0.518
\end{array}
$$

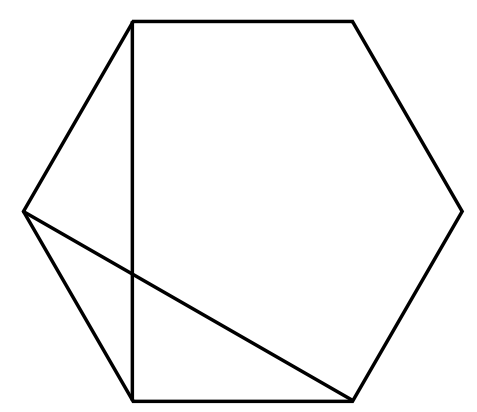

Figure 23: A hexagonal cluster with context $(4,1)$.

9. In this same context, let $F$ be the pentagonal subregion along the upright diagonal. It satisfies

$$
\begin{aligned}
\text { vor }_{0} & <-0.137 \\
\tau_{0} & >0.31
\end{aligned}
$$

10. If the hexagonal cluster has an upright diagonal in context $(4,1)$, and if there is one unmasked flat quarter, let $\{F\}$ be the set of four subregions around the upright diagonal. Assume that the edge opposite the upright diagonal on the anchored simplex has length at least $2 \sqrt{2}$. (There are five subregions, shown in Figure 24.)

$$
\begin{array}{ll}
\operatorname{vor}_{0, R}(D)+\sum_{(3)} \sigma(Q) & <-0.1657 \\
\tau_{0, R}(D)+\sum_{(3)} \tau(Q) & >0.384
\end{array}
$$

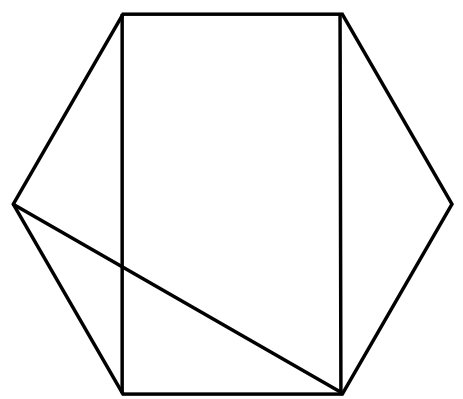

Figure 24: A hexagonal cluster with context $(4,1)$.

11. In this same context, let $F$ be the quadrilateral subregion in Figure 24. It satisfies

$$
\begin{aligned}
& \text { vor }_{0}<-0.084, \\
& \tau_{0}>0.176 .
\end{aligned}
$$

\subsection{Proof of inequalities}

Proposition 18.1. Inequalities 1 -11 are valid.

We prove the inequalities in reverse order 11- 1 . The bounds vor $_{0}<0.009$ and $\tau_{0}>0.05925$ from $\left[6, \mathbf{A}_{13}\right]$ for what a flat quarters with diagonal $\sqrt{8}$ will be used repeatedly. Some of the proofs will make use of tcc-bounds, which are described in $[6$, Section 5.2].

Proof. (Inequality 10 and Inequality 11.) Break the quadrilateral cluster into two simplices $S$ and $S^{\prime}$ along the long edge of the anchored simplex $S$. The anchored simplex $S$ satisfies $\tau(S) \geq 0, \sigma(S) \leq 0$. The other simplex satisfies $\tau_{0}\left(S^{\prime}\right)>0.176$ and $\operatorname{vor}_{0}\left(S^{\prime}\right)<-0.084$ by an interval calculation 18.16. This gives Inequality 11. For Inequality 10, we combine these bounds with the linear programming bound on the four anchored simplices around the upright diagonal. From the inequalities $\left[6, \mathbf{A}_{2}\right]-$ $\left[6, \mathbf{A}_{7}\right],\left[6, \mathbf{A}_{22}\right],\left[6, \mathbf{A}_{24}\right]$, we find that they score $<-0.0817$ and squander $>0.208$. Adding these to the bounds from Inequality 11, we obtain Inequality 10. 
Proof. (Inequality (8) and (9).) The pentagon is a union of an anchored simplex and a quadrilateral region. LP-bounds similar to those in the previous paragraph and based on the inequalities of [6] show that the loop scores at most -0.0817 and squanders at least 0.208. If we show that the quadrilateral satisfies

$$
\begin{aligned}
\operatorname{vor}_{0} & <-0.137 \\
\tau_{0} & >0.31
\end{aligned}
$$

then Inequalities (8) and (9) follow. If by deformations a diagonal of the quadrilateral drops to $2 \sqrt{2}$, then the result follows from Inequalities 18.15 and $\left[6, \mathbf{A}_{13}\right]$. By this we may now assume that the quadrilateral has the form

$$
\left(a_{1}, 2, a_{2}, 2, a_{3}, 2, a_{4}, b_{4}\right), \quad a_{2}, a_{3} \in\left\{2,2 t_{0}\right\} .
$$

If the diagonals drop under 3.2 and $\max \left(a_{2}, a_{3}\right)=$ $2 t_{0}$, again the result follows from Inequalities 18.15 and $\left[6, \mathbf{A}_{13}\right]$. If the diagonals drop under 3.2 and $a_{2}=a_{3}=2$, then the result follows from [6, $\left.\mathbf{A}_{19}\right]$. So finally we attain by deformations $b_{4}=2 \sqrt{2}$ with both diagonals greater than 3.2. But this does not exist, because

$$
\Delta\left(4,4,4,3.2^{2}, 4,8,3.2^{2}\right)<0 .
$$

Proof. (Inequality 5, Inequality 6, and Inequality 7.) Inequalities 7 are derived in [6, Section 5.11]. Inequalities 5,6 are LP-bounds based on $\left[6, \mathbf{A}_{2}\right]$, $\left[6, \mathbf{A}_{7}\right],\left[6, \mathbf{A}_{22}\right]$.

Proof of 4. Deform as in [6]. If at any point a diagonal of the quadrilateral drops to $2 \sqrt{2}$, then the result follows from $\left[6, \mathbf{A}_{13}\right]$ and Inequality 11 :

$$
\begin{aligned}
& \text { vor }_{0} \quad<0.009-0.084=-0.075 \\
& \tau_{0} \quad>0+0.176=0.176
\end{aligned}
$$

Continue deformations until the quadrilateral has the form

$$
\left(a_{1}, 2, a_{2}, 2, a_{3}, 2, a_{4}, b_{4}\right), \quad a_{2}, a_{3} \in\left\{2,2 t_{0}\right\} .
$$

There is necessarily a diagonal of length $\leq 3.2$, because

$$
\Delta\left(4,4,3.2^{2}, 8,4,3.2^{2}\right)<0 .
$$

Suppose the diagonal between vertices $v_{2}$ and $v_{4}$ has length at most 3.2. If $a_{2}=2 t_{0}$ or $a_{3}=2 t_{0}$, the result follows from $\left[6, \mathbf{A}_{13}\right]$ and Inequality 11 . Take $a_{2}=a_{3}=2$. Inequality 4 now follows from $\left[6, \mathbf{A}_{19}\right]$.
Proof. (Inequality 3). We prove that the quadrilateral satisfies

$$
\begin{array}{ll}
\operatorname{vor}_{0} & <-0.168 \\
\tau_{0} & >0.352 .
\end{array}
$$

There are two types of quadrilaterals. In (a), there are two flat quarters whose central vertices are opposite corners of the hexagon. In (b), the flat quarters share a vertex. We consider case (a) first.

Case (a). We deform the quadrilateral as in [6]. If at any point there is a diagonal of length at most 3.2 , the result follows from Inequality 10 and Inequality 11 . Otherwise, the deformations give us a quadrilateral

$$
\left(a_{1}, 2, a_{2}, 2 t_{0}, a_{3}, 2, a_{4}, 2\right), \quad a_{i} \in\left\{2,2 t_{0}\right\} .
$$

The tcc approximation now gives the result (see $[6$, $5.3)]$.

Case (b). Label the vertices of the quadrilateral $v_{1}, \ldots, v_{4}$, where $\left(v_{1}, v_{2}\right)$ and $\left(v_{1}, v_{4}\right)$ are the diagonals of the flat quarter. Again, we deform the quadrilateral. If at any point of the deformation, we find that $\left|v_{1}-v_{3}\right| \leq 3.2$, the result follows from Inequalities 10,11 . If during the deformation $\left|v_{2}-v_{4}\right| \leq 2 \sqrt{2}$, the result follows from $\left[6, \mathbf{A}_{13}\right]$ and the interval calculations 18.14. If the diagonal $\left(v_{2}, v_{4}\right)$ has length at least 3.2 throughout the deformation, we eventually obtain a quadrilateral of the form

$$
\left(a_{1}, 2 t_{0}, a_{2}, 2, a_{3}, 2, a_{4}, 2 t_{0}\right), \quad a_{i} \in\left\{2,2 t_{0}\right\} .
$$

But this does not exist:

$$
\Delta\left(4,4,3.2^{2},\left(2 t_{0}\right)^{2},\left(2 t_{0}\right)^{2}, 3.2^{2}\right)<0 .
$$

We may assume that $\left|v_{2}-v_{4}\right| \in[2 \sqrt{2}, 3.2]$. The result now follows from interval calculations 18.14 .

Proof. (Inequality 2). This case requires more effort. We show that

$$
\begin{array}{ll}
\text { vor }_{0} & <-0.221 \\
\tau_{0} & >0.486
\end{array}
$$

Label the corners $\left(v_{1}, \ldots, v_{5}\right)$ cyclically with $\left(v_{1}, v_{5}\right)$ the diagonal of the flat quarter in the hexagonal cluster. We use the deformation theory of [6]. The proof appears in steps $(1), \ldots,(6)$.

(1) If during the deformations, $\left|v_{1}-v_{4}\right| \leq 3.2$ or $\left|v_{2}-v_{5}\right| \leq 3.2$, the result follows from Inequalities 18.2 and 11 . We may assume this does not occur. 
(2) If an edge $\left(v_{1}, v_{3}\right),\left(v_{2}, v_{4}\right)$, or $\left(v_{3}, v_{5}\right)$ drops to $2 \sqrt{2}$, continue with deformations that do not further decrease this diagonal. If $\left|v_{1}-v_{3}\right|=\left|v_{3}-v_{5}\right|=$ $2 \sqrt{2}$, then the result follows from $\left[6, \mathbf{A}_{13}\right]$ and interval calculations 18.7 .

If we have $\left|v_{1}-v_{3}\right|=2 \sqrt{2}$, deform the figure to the form

$\left(a_{1}, 2, a_{2}, 2, a_{3}, 2, a_{4}, 2, a_{5}, 2 t_{0}\right), \quad a_{2}, a_{4}, a_{5} \in\left\{2,2 t_{0}\right\}$.

Once it is in this form, break the flat quarter $\left(0, v_{1}, v_{2}, v_{3}\right)$ from the cluster and deform $v_{3}$ until $a_{3} \in\left\{2,2 t_{0}\right\}$. The result follows from an interval calculation 18.8.

We handle a boundary case of the preceding calculation separately. After breaking the flat quarter off, we have the cluster

$\left(a_{1}, 2 \sqrt{2}, a_{3}, 2, a_{4}, 2, a_{5}, 2 t_{0}\right), \quad a_{3}, a_{4}, a_{5} \in\left\{2,2 t_{0}\right\}$.

If $\left|v_{1}-v_{4}\right|=3.2$, we break the quadrilateral cluster into two pieces along this diagonal and use interval calculations 18.9 to conclude the result. This completes the analysis of the case $\left|v_{1}-v_{3}\right|=2 \sqrt{2}$.

(3) If $\left|v_{2}-v_{4}\right| \leq 3.2$, then deform until the cluster has the form

$\left(a_{1}, 2, a_{2}, 2, a_{3}, 2, a_{4}, 2, a_{5}, 2 t_{0}\right), \quad a_{1}, a_{3}, a_{5} \in\left\{2,2 t_{0}\right\}$.

Then cut along the special simplex to produce a quadrilateral. Disregarding cases already treated by the interval calculations 18.9 , we can deform it to

$$
\left(a_{1}, 2, a_{2}, 2 \sqrt{2}, a_{4}, 2, a_{5}, 2 t_{0}\right), \quad a_{i} \in\left\{2,2 t_{0}\right\},
$$

with diagonals at least 3.2. The result now follows from the interval calculations 18.10.

In summary of (1), (2), (3), we find that by disregarding cases already considered, we may deform the cluster into the form

$$
\left(a_{1}, 2, a_{2}, 2, a_{3}, 2, a_{4}, 2, a_{5}, 2 t_{0}\right), \quad a_{i} \in\left\{2,2 t_{0}\right\},
$$

$\left|v_{1}-v_{3}\right|>2 \sqrt{2},\left|v_{3}-v_{5}\right|>2 \sqrt{2},\left|v_{2}-v_{4}\right|>3.2$.

(4) Assume $\left|v_{1}-v_{3}\right|,\left|v_{3}-v_{5}\right| \leq 3.2$. If $\max \left(a_{1}, a_{3}, a_{5}\right)=2 t_{0}$, we invoke interval calculations 18.8 and $\left[6, \mathbf{A}_{13}\right]$ to prove the inequalities. So we may assume $a_{1}=a_{3}=a_{5}=2$. The result now follows from interval calculations 18.11. This completes the case $\left|v_{1}-v_{3}\right|,\left|v_{3}-v_{5}\right| \leq 3.2$.

(5) Assume $\left|v_{1}-v_{3}\right|,\left|v_{3}-v_{5}\right| \geq 3.2$. We deform to

$$
\left(a_{1}, 2, a_{2}, 2, a_{3}, 2, a_{4}, 2, a_{5}, 2 t_{0}\right), \quad a_{i} \in\left\{2,2 t_{0}\right\} .
$$

If $a_{2}=2 t_{0}$ and $a_{1}=a_{3}=2$, then the simplex does not exist by Section $[6,5.6]$. Similarly, $a_{4}=2 t_{0}$, $a_{5}=a_{3}=2$ does not exist. The tcc bound gives the result except when $a_{2}=a_{4}=2$. The condition $\left|v_{2}-v_{4}\right| \geq 3.2$ forces $a_{3}=2$. These remaining cases are treated with the interval calculations 18.12.

(6) Assume $\left|v_{1}-v_{3}\right| \leq 3.2$ and $\left|v_{3}-v_{5}\right| \geq 3.2$. This case follows from deformations, interval calculations 18.8, and 18.13. This completes the proof of Inequalities 2.

Proof. (Inequality 1). Label the corners of the hexagon $v_{1}, \ldots, v_{6}$. The proof to this inequality is similar to the other cases. We deform the cluster by the method of IV until it breaks into pieces that are small enough to be estimated by interval calculations. If a diagonal between opposite corners has length at most 3.2, then the hexagon breaks into two quadrilaterals and the result follows from Inequality 18.2 .

If a flat quarter is formed during the course of deformation, then the result follows from Inequality 2 and $\left[6, \mathbf{A}_{13}\right]$. Deform until the hexagon has the form

$$
\left(a_{1}, 2, a_{2}, 2, \ldots, a_{6}, 2\right), \quad a_{i} \in\left\{2,2 t_{0}\right\} .
$$

We may also assume that the hexagon is convex (see [6, Section 4.11]).

If there are no special simplices, we consider the tcc-bound. The tcc-bound implies Inequality 1, except when $a_{i}=2$, for all $i$. But if this occurs, the perimeter of the convex spherical polygon is $6 \operatorname{arc}(2,2,2)=2 \pi$. Thus, there is a pair of antipodal points on the hexagon. The hexagon degenerates to a lune with vertices at the antipodal points. This means that some of the angles of the hexagon are $\pi$. One of the tccs has the form $C(2,1.6, \pi)$, in the notation of [6, Lemma 4.11]. With this extra bit of information, the tcc bound implies Inequality 1.

If there is one special simplex, say $\left|v_{5}-v_{1}\right| \in$ $[2 \sqrt{2}, 3.2]$, we remove it. The score of the special simplex is [6, Inequalities $\mathbf{A}_{13}$ ]

vor $_{0}<0, \quad \tau_{0}>0.05925, \quad$ if $\max \left(\left|v_{1}\right|,\left|v_{5}\right|\right)=2 t_{0}$, vor $_{0}<0.0461, \quad \tau_{0}>0, \quad$ if $\left|v_{1}\right|=\left|v_{5}\right|=2$,

The resulting pentagon can be deformed. If by deformations, we obtain $\left|v_{2}-v_{5}\right|=3.2$ or $\left|v_{1}-v_{4}\right|=$ 3.2 , the result follows from Inequalities 18.2 and the following two interval calculations

vor $_{0}<-0.212-0.0461+0.137$, if $y_{4}=3.2, y_{5} \in$ $[2 \sqrt{2}, 3.2]$.

$(725257062 \dagger)$ 
$\tau_{0}>0.54525-0-0.31$. if $y_{4}=3.2, y_{5} \in[2 \sqrt{2}, 3.2]$.

$(977272202 \dagger)$

If $\left|v_{5}-v_{1}\right|=2 \sqrt{2}$, we use Inequality 2 and $\left[6, \mathbf{A}_{13}\right]$ unless $\left|v_{1}\right|=\left|v_{5}\right|=2$. If $\left|v_{1}\right|=\left|v_{5}\right|=2$, we use the interval calculations 18.3. If a second special simplex forms during the deformations, the result follows from the interval calculations 18.4.

The final case of Inequality 1 to consider is that of two special simplices. We divide this into two cases. (a) The central vertices of the specials are $v_{2}$ and $v_{6}$. (b) The central vertices are opposite $v_{1}$ and $v_{4}$. In case (a), the result follows by deformations and interval calculations 18.5. In case (b), the result follows by deformations and interval calculations 18.6. This completes the proof of Inequalities 1 and the proof of the Proposition.

\subsection{Group $†$}

Let $R$ be a pentagonal region with parameters

$\left(2,2, a_{2}, 2, a_{3}, 2, a_{4}, 2,2,2 \sqrt{2}\right), \quad a_{2}, a_{3}, a_{4} \in\left\{2,2 t_{0}\right\}$

Since this is a pentagonal region, we may discard any edge-length combinations that produce $\Delta<0$. Assume $\left|v_{1}-v_{3}\right| \geq 3.2,\left|v_{3}-v_{5}\right| \geq 3.2$. Under these conditions the following inequalities hold.

vor $_{0}+0.0461<-0.212$

(583626763)

$\tau_{0}>0.54525$

(390951718)

\subsection{Group $\dagger$}

Let $R$ be a pentagonal region with parameters

$$
\left(a_{1}, 2, a_{2}, 2, a_{3}, 2, a_{4}, 2, a_{5}, b_{5}\right), \quad a_{i} \in\left\{2,2 t_{0}\right\} .
$$

Since this is a pentagonal region, we may discard any edge-length combinations that produce $\Delta<0$. Assume $\left|v_{1}-v_{3}\right|,\left|v_{3}-v_{5}\right|, b_{5}=\left|v_{5}-v_{1}\right| \in[2 \sqrt{2}, 3.2]$. Under these conditions the following inequalities hold.

vor $_{0}+0.0461<-0.212$

$(621852152)$

$\tau_{0}>0.54525$

(207203174)

\subsection{Group $\dagger$}

Let $R$ be a hexagonal region with parameters

$$
\left(a_{1}, 2, a_{2}, 2, a_{3}, 2, a_{4}, 2, a_{5}, 2, a_{6}, 2\right), \quad a_{i} \in\left\{2,2 t_{0}\right\} .
$$

Since this is a hexagonal region, we may discard any edge-length combinations that produce $\Delta<0$.
Assume $\left|v_{1}-v_{5}\right|,\left|v_{1}-v_{3}\right| \in[2 \sqrt{2}, 3.2],\left|v_{1}-v_{4}\right| \geq$ $3.2,\left|v_{3}-v_{5}\right| \geq 2 \sqrt{2}$. Under these conditions the following inequalities hold.

$\operatorname{vor}_{0}<-0.212$.

$(368258024)$

$\tau_{0}>0.54525$.

$(564618342)$

\subsection{Group $\dagger$}

Let $R$ be a hexagonal region with parameters

$\left(a_{1}, 2, a_{2}, 2, a_{3}, 2, a_{4}, 2, a_{5}, 2, a_{6}, 2\right), \quad a_{i} \in\left\{2,2 t_{0}\right\}$.

Since this is a hexagonal region, we may discard any edge-length combinations that produce $\Delta<0$. Assume $\left|v_{2}-v_{6}\right|,\left|v_{3}-v_{5}\right| \in[2 \sqrt{2}, 3.2],\left|v_{2}-v_{5}\right| \in$ $[3.2,3.78],\left|v_{3}-v_{6}\right| \geq 3.2$. Under these conditions the following inequalities hold.

vor $_{0}<-0.212$.

(498774382)

$\tau_{0}>0.54525$.

$(544865225)$

\subsection{Group $\dagger$}

vor $_{0}<-0.221-2(0.009)$, if $y_{4}=2 \sqrt{2}, y_{5} \in$ $[2 \sqrt{2}, 3.2]$, and $y_{6} \in\left[2 t_{0}, 2 \sqrt{2}\right] . \quad(234734606 \dagger)$ $\tau_{0}>0.486-2(0.05925)$, if $y_{4}=2 \sqrt{2}, y_{5} \in$ $[2 \sqrt{2}, 3.2], y_{6} \in\left[2 t_{0}, 2 \sqrt{2}\right]$.

$(791682321 \dagger)$

\subsection{Group $\dagger$}

Let $Q$ be a quadrilateral region with parameters

$$
\left(a_{1}, b_{1}, a_{2}, 2, a_{3}, 2, a_{4}, 2 t_{0}\right), \quad a_{2}, a_{3}, a_{4} \in\left\{2,2 t_{0}\right\} .
$$

Since this is a quadrilateral region, we may discard any edge-length combinations that produce $\Delta<0$. Assume $\left|v_{1}-v_{3}\right| \geq 3.2,\left|v_{2}-v_{4}\right| \geq 2 \sqrt{2}$. $b_{1}=$ $\left|v_{1}-v_{2}\right| \in[2 \sqrt{2}, 3.2]$. Under these conditions the following inequalities hold.

$\operatorname{vor}_{0}(Q)<-0.221-0.009$.

(995351614)

$\tau_{0}(Q)>0.486-0.05925$.

(321843503)

\subsection{Group $\dagger$}

vor $_{0}<-0.19-\left(y_{5}-\sqrt{2}\right) 0.14$, if $y_{4}=2, y_{5} \in$ $[2 \sqrt{2}, 3.2]$, and $y_{6} \in[3.2,3.47]$.

(354217730)

$\tau_{0}>0.281$, if $y_{4}=2, y_{5} \in[2 \sqrt{2}, 3.2], y_{6} \in$ $[3.2,3.23]$.

(595674181)

vor $_{0}<-0.11$, if $y_{4}=2, y_{5}=2 t_{0}, y_{6}=3.2$.

$(547486831 \dagger)$ 
$\tau_{0}>0.205$, if $y_{4}=2, y_{5}=2 t_{0}, y_{6}=3.2$.

$(683897354 \dagger)$

vor $_{0}<0.009+\left(y_{5}-2 \sqrt{2}\right) 0.14$, if $y_{5} \in[2 \sqrt{2}, 3.2]$, $y_{4}=y_{6}=2$.

(938003786)

\subsection{Group $\dagger$}

Let $Q$ be a quadrilateral region with parameters

$$
\left(a_{1}, 2, a_{2}, 2 \sqrt{2}, a_{3}, 2, a_{4}, 2 t_{0}\right), \quad a_{i} \in\left\{2,2 t_{0}\right\} .
$$

Since this is a quadrilateral region, we may discard any edge-length combinations that produce $\Delta<0$. Assume $\left|v_{1}-v_{3}\right| \geq 3.2,\left|v_{2}-v_{4}\right| \geq 3.2$. Under these conditions the following inequalities hold.

$\operatorname{vor}_{0}(Q)<-0.221-0.0461$.

(109046923)

$\tau_{0}(Q)>0.486$.

(642590101)

\subsection{Group $\dagger$}

Let $R$ be a pentagonal region with parameters

$$
\left(2,2, a_{2}, 2,2,2, a_{4}, 2,2,2 t_{0}\right), \quad a_{2}, a_{4} \in\left\{2,2 t_{0}\right\} .
$$

Since this is a pentagonal region, we may discard any edge-length combinations that produce $\Delta<0$. Assume $\left|v_{1}-v_{3}\right| \in[2 \sqrt{2}, 3.2],\left|v_{3}-v_{5}\right| \in[2 \sqrt{2}, 3.2]$. Under these conditions the following inequalities hold.

$\operatorname{vor}_{0}(R)<-0.221$.

(160800042)

$\tau_{0}(R)>0.486$.

(690272881)

\subsection{Group $\dagger$}

Let $R$ be a pentagonal region with parameters

$$
\left(a_{1}, 2,2,2,2,2,2,2, a_{5}, 2 t_{0}\right), \quad a_{1}, a_{5} \in\left\{2,2 t_{0}\right\} .
$$

Since this is a pentagonal region, we may discard any edge-length combinations that produce $\Delta<0$. Assume $\left|v_{1}-v_{3}\right| \geq 3.2,\left|v_{3}-v_{5}\right| \geq 3.2$. Assume

$$
\begin{aligned}
\operatorname{dih}\left(0, v_{3}, v_{4}, v_{5}\right) & +\operatorname{dih}\left(0, v_{3}, v_{5}, v_{1}\right)+\operatorname{dih}\left(0, v_{3}, v_{1}, v_{2}\right) \\
& \geq \operatorname{dih}(S(2,2,2,3.2,2,2)) \\
& =\arccos (-53 / 75) .
\end{aligned}
$$

Under these conditions the following inequalities hold.

$\operatorname{vor}_{0}(R)<-0.221$.

(713930036)

$\tau_{0}(R)>0.486$.

(724922588)

\subsection{Group $\dagger$}

Let $R$ be a pentagonal region with parameters

$\left(2,2, a_{2}, 2,2,2, a_{4}, 2, a_{5}, 2 t_{0}\right), \quad a_{2}, a_{4}, a_{5} \in\left\{2,2 t_{0}\right\}$.

Since this is a pentagonal region, we may discard any edge-length combinations that produce $\Delta<$ 0 . Assume $\left|v_{1}-v_{3}\right| \in[2 \sqrt{2}, 3.2],\left|v_{1}-v_{4}\right| \geq 3.2$, $\left|v_{3}-v_{5}\right| \geq 3.2$. Under these conditions the following inequalities hold.

$\operatorname{vor}_{0}(R)<-0.221$.

(821730621)

$\tau_{0}(R)>0.486$.

(890642961)

\subsection{Group $\dagger$}

vor $_{0}<-0.168-0.009, \quad y_{4}=2 \sqrt{2}, y_{5}, y_{6} \in$

$\left[2 t_{0}, 2 \sqrt{2}\right] . \quad(341667126 \dagger)$

$\tau_{0}>0.352-0.05925, y_{4}=2 \sqrt{2}, y_{5}, y_{6} \in\left[2 t_{0}, 2 \sqrt{2}\right]$.

(535906363†)

\subsection{Group}

$\operatorname{vor}_{0}<-0.146$, if $y_{5}, y_{6} \in[2 \sqrt{2}, 3.2] . \quad$ (516537931)

$\tau_{0}\left(S\left(y_{1}, \ldots, y_{6}\right)\right)+\tau_{0}\left(S\left(y_{1}, y_{2}^{\prime}, y_{3}, 2, y_{5}, 2\right)\right)>0.31$, if $y_{5}, y_{6} \in[2 \sqrt{2}, 3.2]$, and $y_{2}^{\prime} \in\left\{2,2 t_{0}\right\}$.

(130008809)

\subsection{Group}

vor $_{0}<-0.084$, if $y_{5} \in\left[2 t_{0}, 2 \sqrt{2}\right], y_{6} \in[2 \sqrt{2}, 3.2]$. (531861442)

vor $_{0}<-0.084-\left(y_{5}-2 \sqrt{2}\right) 0.1$, if $y_{1}=y_{3}=y_{4}=2$, $y_{6}=2 t_{0}, y_{5} \in[2 \sqrt{2}, 3.2]$.

(292827481)

vor $_{0}<0.009+\left(y_{5}-2 \sqrt{2}\right) 0.1$, if $y_{5} \in[2 \sqrt{2}, 3.2]$, $y_{1}=y_{3}=y_{4}=y_{6}=2$.

(710875528)

$\tau_{0}>0.176$, if $y_{5} \in\left[2 t_{0}, 2 \sqrt{2}\right], y_{6} \in[2 \sqrt{2}, 3.2]$.

(286122364) 


\section{References}

[1] T. Hales, An Overview of the Kepler Conjecture, preprint.

[2] S. Ferguson, T. Hales, A Formulation of the Kepler Conjecture, preprint.

[3] Thomas C. Hales, Sphere Packings I, Discrete and Computational Geometry, 17 (1997), 1-51.

[4] Thomas C. Hales, Sphere Packings II, Discrete and Computational Geometry, 18 (1997), 135149.

[5] Thomas C. Hales, Sphere Packings III, preprint.

[6] Thomas C. Hales, Sphere Packings IV, preprint.

[7] S. Ferguson, Sphere Packings V, thesis, University of Michigan, 1997.

[8] Thomas C. Hales, Packings, http://www.math.lsa.umich.edu/ ${ }^{2}$ ales/packings.html

[9] Thomas C. Hales, Some algorithms arising in the proof of the Kepler Conjecture, to appear.

[10] Thomas C. Hales, Remarks on the Density of Sphere Packings, Combinatorica, 13 (2) (1993) 181-197.

[11] Thomas C. Hales, Cannonballs and Honeycombs, Notices of the AMS.

[12] Thomas C. Hales, Sphere Packings in 3 Dimensions, Arbeitstagung, 2001.

This paper was rewritten on $3 / 3 / 02$ from the version of 7/31/98.

Research supported in part by the NSF 